\title{
Twists on the Torus Equivariant under the 2-Dimensional Crystallographic Point Groups
}

Kiyonori GOMI

Department of Mathematical Sciences, Shinshu University, 3-1-1 Asahi, Matsumoto, Nagano 390-8621, Japan

E-mail:kgomi@math.shinshu-u.ac.jp

URL: http://math.shinshu-u.ac.jp/ kgomi/

Received February 17, 2016, in final form March 03, 2017; Published online March 08, 2017

https://doi.org/10.3842/SIGMA.2017.014

\begin{abstract}
A twist is a datum playing a role of a local system for topological $K$-theory. In equivariant setting, twists are classified into four types according to how they are realized geometrically. This paper lists the possible types of twists for the torus with the actions of the point groups of all the 2-dimensional space groups (crystallographic groups), or equivalently, the torus with the actions of all the possible finite subgroups in its mapping class group. This is carried out by computing Borel's equivariant cohomology and the Leray-Serre spectral sequence. As a byproduct, the equivariant cohomology up to degree three is determined in all cases. The equivariant cohomology with certain local coefficients is also considered in relation to the twists of the Freed-Moore $K$-theory.
\end{abstract}

Key words: twist; Borel equivariant cohomology; crystallographic group; topological insulator

2010 Mathematics Subject Classification: 53C08; 55N91; 20H15; 81T45

\section{Introduction}

Topological $K$-theory has recently been recognized as a useful tool for a classification of topological insulators in condensed matter physics. In Kitaev's 10-fold way [17], the usual complex $K$-theory and also $K O$ or Atiyah's $K R$-theory are used. These classifications are in some sense the most simple cases, and a recent study of topological insulators focuses on more complicated cases. Such complicated cases arise when we take the symmetry of quantum systems into account. Then equivariant $K$-theory and its twisted version naturally fit into the classification scheme of such systems [8]. Actually, as will be explained in Section 2, a certain quantum system on the $d$-dimensional space $\mathbb{R}^{d}$ invariant under a space group provides a $K$-theory class on the $d$-dimensional torus $T^{d}$ equivariant under the point group of the space group. If the space group is nonsymmorphic, then the equivariant $K$-class is naturally twisted. In the case of $d=2$, such (twisted) equivariant $K$-theories are computed for the 17 classes of 2 -dimensional space groups, in view of the classification of topological crystalline insulators [27, 28]. An outcome of these computations of twisted equivariant $K$-theories is the discovery of topological insulators which are essentially classified by $\mathbb{Z}_{2}$ but do not require the so-called time-reversal symmetry or the particle-hole symmetry [26]. This type of topological insulators is new in the sense that the known topological insulators essentially classified by $\mathbb{Z}_{2}$ so far require the time-reversal symmetry or the particle-hole symmetry.

The understanding of the importance of twisted equivariant $K$-theory in condensed matter physics leads to a mathematically natural issue: determining the possible 'twists' for equivariant $K$-theory. To explain this issue more concretely, let us recall that twisted $K$-theory $[5,22]$ is in some sense a $K$-theory with 'local coefficients'. The datum playing the role of a 'local system' 
admits various geometric realizations. In this paper, we realize them by twists in the sense of [7]. If a compact Lie group $G$ acts on a space $X$, then graded twists on $X$ are classified by the Borel equivariant cohomology $H_{G}^{1}\left(X ; \mathbb{Z}_{2}\right) \times H_{G}^{3}(X ; \mathbb{Z})$. Similarly, ungraded twists are classified by $H_{G}^{3}(X ; \mathbb{Z})$, on which we focus for a moment. (Sometimes $H_{G}^{0}(X ; \mathbb{Z})$ may be included in the twists, but we regard it as the degree of the $K$-theory.)

By definition, the Borel equivariant cohomology $H_{G}^{n}(X ; \mathbb{Z})$ is the usual cohomology $H^{n}\left(E G \times_{G}\right.$ $X ; \mathbb{Z})$ of the Borel construction $E G \times{ }_{G} X$, which is the quotient of $E G \times X$ by the diagonal $G$-action, where $E G$ is the total space of the universal $G$-bundle $E G \rightarrow B G$. Associated to the Borel construction is the fibration $X \rightarrow E G \times_{G} X \rightarrow B G$, and hence the Leray-Serre spectral sequence $E_{r}^{p, q}$ that converges to the graded quotient of a filtration

$$
H_{G}^{n}(X ; \mathbb{Z}) \supset F^{1} H_{G}^{n}(X ; \mathbb{Z}) \supset F^{2} H_{G}^{n}(X ; \mathbb{Z}) \supset \cdots \supset F^{n+1} H_{G}^{n}(X ; \mathbb{Z})=0 .
$$

One can interpret $F^{p} H_{G}^{3}(X ; \mathbb{Z}) \subset H_{G}^{3}(X ; \mathbb{Z})$ geometrically in the classification of twists, and there are four types (see Section 3 for details):

(i) Twists which can be represented by group 2-cocycles of $G$ with coefficients in the trivial $G$-module $U(1)$. These twists are classified by $F^{3} H_{G}^{3}(X ; \mathbb{Z})$.

(ii) Twists which can be represented by group 2-cocycles of $G$ with coefficients in the group $C(X, U(1))$ of $U(1)$-valued functions on $X$ regarded as a (right) $G$-module by pull-back. These twists are classified by $F^{2} H_{G}^{3}(X ; \mathbb{Z})$.

(iii) Twists which can be represented by central extensions of the groupoid $X / / G$. These twists are classified by $F^{1} H_{G}^{3}(X ; \mathbb{Z})$.

(iv) Twists of general type, classified by $F^{0} H_{G}^{3}(X ; \mathbb{Z})=H_{G}^{3}(X ; \mathbb{Z})$.

The equivariant twists on $T^{d}$ arising from quantum systems on $\mathbb{R}^{d}$, to be explained in Section 2 , belong to $F^{2} H_{P}^{3}\left(T^{d} ; \mathbb{Z}\right)$ with $P$ the point group of a $d$-dimensional space group $S$, and so are the twists considered in [27]. Now, the mathematical issue is whether the twists arising in this way cover all the possibilities or not. The present paper answers this question in the case of $d=2$ by a theorem (Theorem 1.1).

To state the theorem, let $S$ be a 2-dimensional space group, which is also known as a 2dimensional crystallographic group, a plane symmetry group, a wallpaper group, and so on. It is a subgroup of the Euclidean group $\mathbb{R}^{2} \rtimes \mathrm{O}(2)$ of isometries of $\mathbb{R}^{2}$, and is an extension of a finite group $P \subset \mathrm{O}(2)$ called the point group by a rank 2 lattice $\Pi \cong \mathbb{Z}^{2}$ of translations of $\mathbb{R}^{2}$ :

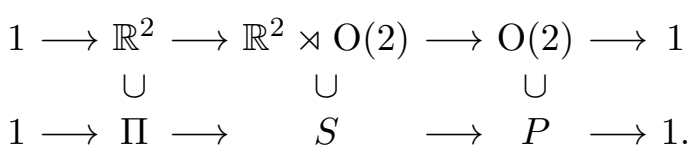

Being a normal subgroup of $S$, the lattice $\Pi \subset \mathbb{R}^{2}$ is preserved by the action of $P$ on $\mathbb{R}^{2}$ through the inclusion $P \subset \mathrm{O}(2)$ and the standard left action of $\mathrm{O}(2)$ on $\mathbb{R}^{2}$. This induces the left action of $P$ on the torus $T^{2}=\mathbb{R}^{2} / \Pi$ that we will consider. Since $P$ is a finite subgroup of $\mathrm{O}(2)$, it is the cyclic group $\mathbb{Z}_{n}$ of order $n$ or the dihedral group $D_{n}=\left\langle C, \sigma \mid C^{n}, \sigma^{2}, \sigma C \sigma C\right\rangle$ of degree $n$ and order $2 n$. The classification of 2-dimensional space groups has long been known, and there are 17 types [12, 24], which we label following [23]. Notice that some space groups share the same point group action on $T^{2}$, and there arise 13 distinct finite group actions on the torus. These actions realize essentially all the possible finite subgroups in the mapping class group of the torus [20], which is isomorphic to $\mathrm{GL}(2, \mathbb{Z})$ as is well known [21].

Theorem 1.1. Let $P$ be the point group of one of the 2-dimensional space groups $S$, acting on $T^{2}=\mathbb{R}^{2} / \Pi$ via $P \subset \mathrm{O}(2)$ as above. Then, $H_{P}^{3}\left(T^{2} ; \mathbb{Z}\right)=F^{0} H_{P}^{3}\left(T^{2} ; \mathbb{Z}\right)=F^{1} H_{P}^{3}\left(T^{2} ; \mathbb{Z}\right)$. This cohomology group and its subgroups $F^{p} H_{P}^{3}\left(T^{2} ; \mathbb{Z}\right)$ are as in Fig. 1. 


\begin{tabular}{|c|c|c||c|c|c||c|c|}
\hline Space group $S$ & $P$ & ori & $H_{P}^{3}\left(T^{2} ; \mathbb{Z}\right)$ & $F^{2}$ & $F^{3}$ & $E_{\infty}^{1,2}$ & $E_{\infty}^{2,1}$ \\
\hline $\mathrm{p} 1$ & 1 & + & 0 & 0 & 0 & 0 & 0 \\
\hline $\mathrm{p} 2$ & $\mathbb{Z}_{2}$ & + & 0 & 0 & 0 & 0 & 0 \\
\hline $\mathrm{p} 3$ & $\mathbb{Z}_{3}$ & + & 0 & 0 & 0 & 0 & 0 \\
\hline $\mathrm{p} 4$ & $\mathbb{Z}_{4}$ & + & 0 & 0 & 0 & 0 & 0 \\
\hline $\mathrm{p} 6$ & $\mathbb{Z}_{6}$ & + & 0 & 0 & 0 & 0 & 0 \\
\hline $\mathrm{pm} / \mathrm{pg}$ & $D_{1}$ & - & $\mathbb{Z}_{2}^{\oplus 2}$ & $\mathbb{Z}_{2}$ & 0 & $\mathbb{Z}_{2}$ & $\mathbb{Z}_{2}$ \\
\hline $\mathrm{cm}$ & $D_{1}$ & - & $\mathbb{Z}_{2}$ & 0 & 0 & $\mathbb{Z}_{2}$ & 0 \\
\hline $\mathrm{pmm} / \mathrm{pmg} / \mathrm{pgg}$ & $D_{2}$ & - & $\mathbb{Z}_{2}^{\oplus 4}$ & $\mathbb{Z}_{2}^{\oplus 3}$ & $\mathbb{Z}_{2}$ & $\mathbb{Z}_{2}$ & $\mathbb{Z}_{2}^{\oplus 2}$ \\
\hline $\mathrm{cmm}$ & $D_{2}$ & - & $\mathbb{Z}_{2}^{\oplus 2}$ & $\mathbb{Z}_{2}$ & $\mathbb{Z}_{2}$ & $\mathbb{Z}_{2}$ & 0 \\
\hline $\mathrm{p} 3 \mathrm{~m} 1$ & $D_{3}$ & - & $\mathbb{Z}_{2}$ & 0 & 0 & $\mathbb{Z}_{2}$ & 0 \\
\hline $\mathrm{p} 31 \mathrm{~m}$ & $D_{3}$ & - & $\mathbb{Z}_{2}$ & 0 & 0 & $\mathbb{Z}_{2}$ & 0 \\
\hline $\mathrm{p} 4 \mathrm{~m} / \mathrm{p} 4 \mathrm{~g}$ & $D_{4}$ & - & $\mathbb{Z}_{2}^{\oplus 3}$ & $\mathbb{Z}_{2}^{\oplus 2}$ & $\mathbb{Z}_{2}$ & $\mathbb{Z}_{2}$ & $\mathbb{Z}_{2}$ \\
\hline $\mathrm{p} 6 \mathrm{~m}$ & $D_{6}$ & - & $\mathbb{Z}_{2}^{\oplus 2}$ & $\mathbb{Z}_{2}$ & $\mathbb{Z}_{2}$ & $\mathbb{Z}_{2}$ & 0 \\
\hline
\end{tabular}

Figure 1. The list of $H_{P}^{3}\left(T^{2} ; \mathbb{Z}\right)$ and its subgroups $F^{p}=F^{p} H_{P}^{3}\left(T^{2} ; \mathbb{Z}\right)$ for the point group $P$ of each space 2-dimensional space group $S$. The $E_{\infty}$-term of the Leray-Serre spectral sequence is related to these subgroups by $E_{\infty}^{p, 3-p} \cong F^{p} / F^{p+1}$. The column "ori" indicates "+" if $P$ preserves the orientation of $T^{2}$ and "-" if not. The same actions of point groups on $T^{2}$ are grouped in a row. Nonsymmorphic groups are pg, pmg, pgg and p4g.

Corollary 1.2. Under the same hypothesis as in Theorem 1.1,

(a) All the twists can be represented by central extensions of $T^{2} / / P$. In particular, there is no non-trivial twist if $P$ preserves the orientation of $T^{2}$.

(b) If $P$ does not preserve the orientation of $T^{2}$, then there are twists which can be represented by central extensions of $T^{2} / / P$ but not by group 2-cocycles of $P$.

(c) The subgroup $F^{2} H_{P}^{3}\left(T^{2} ; \mathbb{Z}\right)$ is generated by the twists represented by:

- group 2-cocycle of $P$ with values in $C\left(T^{2}, U(1)\right)$ induced from a nonsymmorphic space group $S^{\prime}$ such that the action of its point group $P^{\prime} \cong P$ on $T^{2}$ is the same as $P$; and

- group 2-cocycle of $P$ with values in $U(1)$.

As a result, all the twists classified by $F^{2} H_{P}^{3}\left(T^{2} ; \mathbb{Z}\right)$ are relevant to topological insulators, whereas there actually exist other twists which cannot be realized by group cocycles. At present their roles in condensed matter theory seem to be unknown.

Theorem 1.1 follows from case by case computations of the equivariant cohomology $H_{P}^{3}\left(T^{2} ; \mathbb{Z}\right)$ and the Leray-Serre spectral sequence. Roughly, there are three methods according to the nature of the point group actions: The first method is applied to the cases where the torus $T^{2}$ is the product of circles with $P$-actions, i.e., the cases of the $\mathbb{Z}_{2}$-actions arising from $\mathrm{p} 2$ and $\mathrm{pm} / \mathrm{pg}$. In these cases, the equivariant cohomology is computed by means of the splitting of the Gysin exact sequence, as detailed in [10]. The second method is applied to the cases where the point group has no element of order 3 . In these cases, the torus $T^{2}$ admits an equivariant stable splitting. As a result, the equivariant cohomology of $T^{2}$ admits the corresponding splitting, and the Leray-Serre spectral sequence turns out to be trivial. Finally, the third method is applied 
to the remaining cases. In these cases, we take a $P$-CW decomposition of $T^{2}$ to compute the equivariant cohomology by using the Mayer-Vietoris exact sequence and the exact sequence for a pair, and then study the Leray-Serre spectral sequence. In principle, the third method is the most basic, and hence is applied to all the cases. However, to simplify the computations, we use other methods.

These computations contain enough information to determine the equivariant cohomology $H_{P}^{n}\left(T^{2} ; \mathbb{Z}\right),(n \leq 2)$ of the torus with the actions of the possible finite subgroups in the mapping class group $\mathrm{GL}(2, \mathbb{Z})$.

Theorem 1.3. Let $P$ be the point group of one of the 2-dimensional space groups $S$, acting on $T^{2}=\mathbb{R}^{2} / \Pi$ via $P \subset \mathrm{O}(2)$. For $n \leq 3$, the $P$-equivariant cohomology $H_{P}^{n}\left(T^{2} ; \mathbb{Z}\right)$ is as given in Fig. 2.

\begin{tabular}{|c|c|c||c|c|c|c|}
\hline Space group $S$ & $P$ & ori & $H_{P}^{0}\left(T^{2}\right)$ & $H_{P}^{1}\left(T^{2}\right)$ & $H_{P}^{2}\left(T^{2}\right)$ & $H_{P}^{3}\left(T^{2}\right)$ \\
\hline p1 & 1 & + & $\mathbb{Z}$ & $\mathbb{Z}^{\oplus 2}$ & $\mathbb{Z}$ & 0 \\
\hline p2 & $\mathbb{Z}_{2}$ & + & $\mathbb{Z}$ & 0 & $\mathbb{Z} \oplus \mathbb{Z}_{2}^{\oplus 3}$ & 0 \\
\hline $\mathrm{p} 3$ & $\mathbb{Z}_{3}$ & + & $\mathbb{Z}$ & 0 & $\mathbb{Z} \oplus \mathbb{Z}_{3}^{\oplus 2}$ & 0 \\
\hline p4 & $\mathbb{Z}_{4}$ & + & $\mathbb{Z}$ & 0 & $\mathbb{Z} \oplus \mathbb{Z}_{2} \oplus \mathbb{Z}_{4}$ & 0 \\
\hline $\mathrm{p} 6$ & $\mathbb{Z}_{6}$ & + & $\mathbb{Z}$ & 0 & $\mathbb{Z} \oplus \mathbb{Z}_{6}$ & 0 \\
\hline $\mathrm{pm} / \mathrm{pg}$ & $D_{1}$ & - & $\mathbb{Z}$ & $\mathbb{Z}$ & $\mathbb{Z}_{2}^{\oplus 2}$ & $\mathbb{Z}_{2}^{\oplus 2}$ \\
\hline $\mathrm{cm}$ & $D_{1}$ & - & $\mathbb{Z}$ & $\mathbb{Z}$ & $\mathbb{Z}_{2}$ & $\mathbb{Z}_{2}$ \\
\hline $\mathrm{pmm} / \mathrm{pmg} / \mathrm{pgg}$ & $D_{2}$ & - & $\mathbb{Z}$ & 0 & $\mathbb{Z}_{2}^{\oplus 4}$ & $\mathbb{Z}_{2}^{\oplus 4}$ \\
\hline $\mathrm{cmm}$ & $D_{2}$ & - & $\mathbb{Z}$ & 0 & $\mathbb{Z}_{2}^{\oplus 3}$ & $\mathbb{Z}_{2}^{\oplus 2}$ \\
\hline $\mathrm{p} 3 \mathrm{~m} 1$ & $D_{3}$ & - & $\mathbb{Z}$ & 0 & $\mathbb{Z}_{2}$ & $\mathbb{Z}_{2}$ \\
\hline $\mathrm{p} 31 \mathrm{~m}$ & $D_{3}$ & - & $\mathbb{Z}$ & 0 & $\mathbb{Z}_{3} \oplus \mathbb{Z}_{2}$ & $\mathbb{Z}_{2}$ \\
\hline $\mathrm{p} 4 \mathrm{~m} / \mathrm{p} 4 \mathrm{~g}$ & $D_{4}$ & - & $\mathbb{Z}$ & 0 & $\mathbb{Z}_{2}^{\oplus 3}$ & $\mathbb{Z}_{2}^{\oplus 3}$ \\
\hline $\mathrm{p} 6 \mathrm{~m}$ & $D_{6}$ & - & $\mathbb{Z}$ & 0 & $\mathbb{Z}_{2}^{\oplus 2}$ & $\mathbb{Z}_{2}^{\oplus 2}$ \\
\hline
\end{tabular}

Figure 2. The list of equivariant cohomology up to degree 3 .

Note that some specific cases are computed in the literature (e.g., [1, 2, 3]).

So far we focused on ungraded twists. To complete the classification of $P$-equivariant twists on $T^{2}$, we need to compute the equivariant first cohomology with coefficients in $\mathbb{Z}_{2}$, which provides the information on 'gradings' of a twist. But, the computation is immediately completed by a simple application of the universal coefficient theorem to Theorem 1.3. Notice that the equivariant cohomology $H_{P}^{1}\left(T^{2} ; \mathbb{Z}_{2}\right)$ also admits a filtration

$$
H_{P}^{1}\left(T^{2} ; \mathbb{Z}_{2}\right)=F^{0} H_{P}^{1}\left(T^{2} ; \mathbb{Z}_{2}\right) \supset F^{1} H_{P}^{1}\left(T^{2} ; \mathbb{Z}_{2}\right) \supset F^{2} H_{P}^{1}\left(T^{2} ; \mathbb{Z}_{2}\right)=0 .
$$

Because the degree in question is 1, the degeneration of the Leray-Serre spectral sequence gives the identification

$$
F^{1} H_{P}^{1}\left(T^{2} ; \mathbb{Z}_{2}\right)=\operatorname{Hom}\left(P, \mathbb{Z}_{2}\right)=H_{P}^{1}\left(\mathrm{pt} ; \mathbb{Z}_{2}\right),
$$

which is a direct summand of $H_{P}^{1}\left(T^{2} ; \mathbb{Z}_{2}\right)$ and is also computed immediately by using the knowledge of the equivariant cohomology of the space consisting of one point, $\mathrm{pt}=\{$ one point $\}$, in Section 4.1. 
Corollary 1.4. Let $P$ be the point group of one of the 2-dimensional space groups $S$, acting on $T^{2}=\mathbb{R}^{2} / \Pi$ via $P \subset \mathrm{O}(2)$. Then the $P$-equivariant cohomology $H_{P}^{1}\left(T^{2} ; \mathbb{Z}_{2}\right)$ is as in Fig. 3 .

\begin{tabular}{|c|c|c||c|c|c|}
\hline Space group $S$ & $P$ & ori & $H_{P}^{1}\left(T^{2} ; \mathbb{Z}_{2}\right)$ & $F^{1} H_{P}^{1}\left(T^{2} ; \mathbb{Z}_{2}\right)$ & $E_{\infty}^{1,0}$ \\
\hline p1 & 1 & + & $\mathbb{Z}_{2}^{\oplus 2}$ & 0 & $\mathbb{Z}_{2}^{\oplus 2}$ \\
\hline p2 & $\mathbb{Z}_{2}$ & + & $\mathbb{Z}_{2}^{\oplus 3}$ & $\mathbb{Z}_{2}$ & $\mathbb{Z}_{2}^{\oplus 2}$ \\
\hline p3 & $\mathbb{Z}_{3}$ & + & 0 & 0 & 0 \\
\hline p4 & $\mathbb{Z}_{4}$ & + & $\mathbb{Z}_{2}^{\oplus 2}$ & $\mathbb{Z}_{2}$ & $\mathbb{Z}_{2}$ \\
\hline p6 & $\mathbb{Z}_{6}$ & + & $\mathbb{Z}_{2}$ & $\mathbb{Z}_{2}$ & 0 \\
\hline $\mathrm{pm} / \mathrm{pg}$ & $D_{1}$ & - & $\mathbb{Z}_{2}^{\oplus 3}$ & $\mathbb{Z}_{2}$ & $\mathbb{Z}_{2}^{\oplus 2}$ \\
\hline $\mathrm{cm}$ & $D_{1}$ & - & $\mathbb{Z}_{2}^{\oplus 2}$ & $\mathbb{Z}_{2}$ & $\mathbb{Z}_{2}$ \\
\hline $\mathrm{pmm} / \mathrm{pmg} / \mathrm{pgg}$ & $D_{2}$ & - & $\mathbb{Z}_{2}^{\oplus 4}$ & $\mathbb{Z}_{2}^{\oplus 2}$ & $\mathbb{Z}_{2}^{\oplus 2}$ \\
\hline $\mathrm{cmm}$ & $D_{2}$ & - & $\mathbb{Z}_{2}^{\oplus 3}$ & $\mathbb{Z}_{2}^{\oplus 2}$ & $\mathbb{Z}_{2}$ \\
\hline $\mathrm{p} 3 \mathrm{~m} 1$ & $D_{3}$ & - & $\mathbb{Z}_{2}$ & $\mathbb{Z}_{2}$ & 0 \\
\hline $\mathrm{p} 31 \mathrm{~m}$ & $D_{3}$ & - & $\mathbb{Z}_{2}$ & $\mathbb{Z}_{2}$ & 0 \\
\hline $\mathrm{p} 4 \mathrm{~m} / \mathrm{p} 4 \mathrm{~g}$ & $D_{4}$ & - & $\mathbb{Z}_{2}^{\oplus 3}$ & $\mathbb{Z}_{2}^{\oplus 2}$ & $\mathbb{Z}_{2}$ \\
\hline $\mathrm{p} 6 \mathrm{~m}$ & $D_{6}$ & - & $\mathbb{Z}_{2}^{\oplus 2}$ & $\mathbb{Z}_{2}^{\oplus 2}$ & 0 \\
\hline
\end{tabular}

Figure 3. The list of first equivariant cohomology groups with coefficients $\mathbb{Z}_{2}$. The quotient group $H_{P}^{1}\left(T^{2} ; \mathbb{Z}_{2}\right) / F^{1} H_{P}^{1}\left(T^{2} ; \mathbb{Z}_{2}\right)$ is denoted with $E_{\infty}^{1,0}$.

The grading of twists classified by $F^{1} H_{P}^{1}\left(T^{2} ; \mathbb{Z}_{2}\right)=\operatorname{Hom}\left(P, \mathbb{Z}_{2}\right)$ plays a role in a quantum system with symmetry (see Remark 2.2). However, there are other gradings generally, and their roles in condensed matter theory is unknown.

As is mentioned, Atiyah's $K R$-theory is also applied to the classification of topological insulators. The symmetry of $K R$-theory however concerns $\mathbb{Z}_{2}$-actions only, and its use is limited to rather simple cases. To take more general symmetries into account, Freed and Moore introduced a $K$-theory which unifies $K R$-theory and equivariant $K$-theory [8]. Their $K$-theory is defined for a space $X$ with an action of a compact Lie group $G$ equipped with a homomorphism $\phi: G \rightarrow \mathbb{Z}_{2}$. The $K$-theory of Freed-Moore reduces to the $G$-equivariant $K$-theory if $\phi$ is trivial, and to the $K R$-theory if $G=\mathbb{Z}_{2}$ and $\phi$ non-trivial. There also exists the notion of twists for the Freed-Moore $K$-theory. A computation of the twisted Freed-Moore $K$-theory is carried out in [27], leading to the discovery of a novel $\mathbb{Z}_{4}$-phase.

The knowledge about the twists of the Freed-Moore $K$-theory has therefore potential importance to condensed matter physics as well, and the present paper provides it also in the case where $X$ is the torus $T^{2}$ and $G$ is the point group $P$ of a 2 -dimensional space group. Notice that the classification of the twists for the Freed-Moore $K$-theory parallels that of the twists for equivariant $K$-theory (actually a generalization). In general, the graded twists are classified by $H_{G}^{1}\left(X ; \mathbb{Z}_{2}\right) \times H_{G}^{3}\left(X ; \mathbb{Z}_{\phi}\right)$ and the ungraded twists by $H_{G}^{3}\left(X ; \mathbb{Z}_{\phi}\right)$. Here $\mathbb{Z}_{\phi}$ denotes a local system for the Borel equivariant cohomology associated to the $G$-module $\mathbb{Z}_{\phi}$ such that its underlying group is $\mathbb{Z}$ and $G$ acts via $\phi: G \rightarrow \mathbb{Z}_{2}$. The cohomology group $H_{G}^{n}\left(X ; \mathbb{Z}_{\phi}\right)$ also admits a filtration

$$
H_{G}^{n}\left(X ; \mathbb{Z}_{\phi}\right) \supset F^{1} H_{G}^{n}\left(X ; \mathbb{Z}_{\phi}\right) \supset F^{2} H_{G}^{n}\left(X ; \mathbb{Z}_{\phi}\right) \supset \cdots \supset F^{n+1} H_{G}^{n}\left(X ; \mathbb{Z}_{\phi}\right)=0 .
$$

The associated graded quotient is computed by the Leray-Serre spectral sequence, and the subgroups $F^{p} H_{G}^{3}\left(X ; \mathbb{Z}_{\phi}\right) \subset H_{G}^{3}\left(X ; \mathbb{Z}_{\phi}\right)$ have geometric interpretations as well (Proposition 5.1). 
To state our results in the 'twisted' case, we introduce the following definition for the point group $P$ of a 2-dimensional space group $S$ that admits a non-trivial homomorphism $\phi: P \rightarrow \mathbb{Z}_{2}$.

- In the cases of p2, p4 and p6, the point group $P$ is the cyclic group $\mathbb{Z}_{2 m}=\left\langle C \mid C^{2 m}\right\rangle$ of even order. We write $\phi_{1}: \mathbb{Z}_{2 m} \rightarrow \mathbb{Z}_{2}$ for the unique non-trivial homomorphism given by $\phi_{1}(C)=-1$.

- In the other case, the point group $P$ is the dihedral group $D_{n}=\left\langle C, \sigma \mid C^{n}, \sigma^{2}, \sigma C \sigma C\right\rangle$ of degree $n$ and order $2 n$, and $D_{n}$ is embedded into $\mathrm{O}(2)$ so that $C$ is a rotation of $\mathbb{R}^{2}$ and $\sigma$ is a reflection. We define $\phi_{0}: D_{n} \rightarrow \mathbb{Z}_{2}$ to be the composition of the inclusion $D_{n} \rightarrow \mathrm{O}(2)$ and det: $\mathrm{O}(2) \rightarrow \mathbb{Z}_{2}$. Put differently, $\phi_{0}(C)=1$ and $\phi_{0}(\sigma)=-1$. This provides the unique non-trivial homomorphism $D_{n} \rightarrow \mathbb{Z}_{2}$ if $n$ is odd. In the case of even $n$, we define two more non-trivial homomorphisms $\phi_{i}: D_{n} \rightarrow \mathbb{Z}_{2}$ by

$$
\left\{\begin{array} { l } 
{ \phi _ { 1 } ( C ) = - 1 , } \\
{ \phi _ { 1 } ( \sigma ) = 1 , }
\end{array} \quad \left\{\begin{array}{l}
\phi_{2}(C)=-1, \\
\phi_{2}(\sigma)=-1 .
\end{array}\right.\right.
$$

Theorem 1.5. Let $P$ be the point group of one of the 2-dimensional space groups $S$, acting on $T^{2}=\mathbb{R}^{2} / \Pi$ via $P \subset \mathrm{O}(2)$, and $\phi: P \rightarrow \mathbb{Z}_{2}$ a non-trivial homomorphism. Then, $H_{P}^{3}\left(T^{2} ; \mathbb{Z}_{\phi}\right)=$ $F^{0} H_{P}^{3}\left(T^{2} ; \mathbb{Z}_{\phi}\right)=F^{1} H_{P}^{3}\left(T^{2} ; \mathbb{Z}_{\phi}\right)$. This cohomology group and its subgroups $F^{p} H_{P}^{3}\left(T^{2} ; \mathbb{Z}_{\phi}\right)$ are as in Fig. 4.

\begin{tabular}{|c|c|c||c|c|c||c|c|}
\hline Space group $S$ & $P$ & $\phi$ & $H_{P}^{3}\left(T^{2} ; \mathbb{Z}_{\phi}\right)$ & $F^{2}$ & $F^{3}$ & $E_{\infty}^{1,2}$ & $E_{\infty}^{2,1}$ \\
\hline p2 & $\mathbb{Z}_{2}$ & $\phi_{1}$ & $\mathbb{Z}_{2}^{\oplus 4}$ & $\mathbb{Z}_{2}^{\oplus 3}$ & $\mathbb{Z}_{2}$ & $\mathbb{Z}_{2}$ & $\mathbb{Z}_{2}^{\oplus 2}$ \\
\hline p4 & $\mathbb{Z}_{4}$ & $\phi_{1}$ & $\mathbb{Z}_{2}^{\oplus 2}$ & $\mathbb{Z}_{2}$ & $\mathbb{Z}_{2}$ & $\mathbb{Z}_{2}$ & 0 \\
\hline p6 & $\mathbb{Z}_{6}$ & $\phi_{1}$ & $\mathbb{Z}_{2}^{\oplus 2}$ & $\mathbb{Z}_{2}$ & $\mathbb{Z}_{2}$ & $\mathbb{Z}_{2}$ & 0 \\
\hline $\mathrm{pm} / \mathrm{pg}$ & $D_{1}$ & $\phi_{0}$ & $\mathbb{Z}_{2}^{\oplus 2}$ & $\mathbb{Z}_{2}^{\oplus 2}$ & $\mathbb{Z}_{2}$ & 0 & $\mathbb{Z}_{2}$ \\
\hline $\mathrm{cm}$ & $D_{1}$ & $\phi_{0}$ & $\mathbb{Z}_{2}$ & $\mathbb{Z}_{2}$ & $\mathbb{Z}_{2}$ & 0 & 0 \\
\hline $\mathrm{pmm} / \mathrm{pmg} / \mathrm{pgg}$ & $D_{2}$ & $\phi_{0}$ & $\mathbb{Z}_{2}^{\oplus 4}$ & $\mathbb{Z}_{2}^{\oplus 4}$ & $\mathbb{Z}_{2}^{\oplus 2}$ & 0 & $\mathbb{Z}_{2}^{\oplus 2}$ \\
\hline $\mathrm{pmm} / \mathrm{pmg} / \mathrm{pgg}$ & $D_{2}$ & $\phi_{1}, \phi_{2}$ & $\mathbb{Z}_{2}^{\oplus 6}$ & $\mathbb{Z}_{2}^{\oplus 5}$ & $\mathbb{Z}_{2}^{\oplus 2}$ & $\mathbb{Z}_{2}$ & $\mathbb{Z}_{2}^{\oplus 3}$ \\
\hline $\mathrm{cmm}$ & $D_{2}$ & $\phi_{0}$ & $\mathbb{Z}_{2}^{\oplus 2}$ & $\mathbb{Z}_{2}^{\oplus 2}$ & $\mathbb{Z}_{2}^{\oplus 2}$ & 0 & 0 \\
\hline $\mathrm{cmm}$ & $D_{2}$ & $\phi_{1}, \phi_{2}$ & $\mathbb{Z}_{2}^{\oplus 4}$ & $\mathbb{Z}_{2}^{\oplus 3}$ & $\mathbb{Z}_{2}^{\oplus 2}$ & $\mathbb{Z}_{2}$ & $\mathbb{Z}_{2}$ \\
\hline $\mathrm{p} 3 \mathrm{~m} 1$ & $D_{3}$ & $\phi_{0}$ & $\mathbb{Z}_{2}$ & $\mathbb{Z}_{2}$ & $\mathbb{Z}_{2}$ & 0 & 0 \\
\hline $\mathrm{p} 31 \mathrm{~m}$ & $D_{3}$ & $\phi_{0}$ & $\mathbb{Z}_{2}$ & $\mathbb{Z}_{2}$ & $\mathbb{Z}_{2}$ & 0 & 0 \\
\hline $\mathrm{p} 4 \mathrm{~m} / \mathrm{p} 4 \mathrm{~g}$ & $D_{4}$ & $\phi_{0}$ & $\mathbb{Z}_{2}^{\oplus 3}$ & $\mathbb{Z}_{2}^{\oplus 3}$ & $\mathbb{Z}_{2}^{\oplus 2}$ & 0 & $\mathbb{Z}_{2}$ \\
\hline $\mathrm{p} 4 \mathrm{~m} / \mathrm{p} 4 \mathrm{~g}$ & $D_{4}$ & $\phi_{1}, \phi_{2}$ & $\mathbb{Z}_{2}^{\oplus 4}$ & $\mathbb{Z}_{2}^{\oplus 3}$ & $\mathbb{Z}_{2}^{\oplus 2}$ & $\mathbb{Z}_{2}$ & $\mathbb{Z}_{2}$ \\
\hline $\mathrm{p} 6 \mathrm{~m}$ & $D_{6}$ & $\phi_{0}$ & $\mathbb{Z}_{2}^{\oplus 2}$ & $\mathbb{Z}_{2}^{\oplus 2}$ & $\mathbb{Z}_{2}^{\oplus 2}$ & 0 & 0 \\
\hline $\mathrm{p} 6 \mathrm{~m}$ & $D_{6}$ & $\phi_{1}$ & $\mathbb{Z}_{2}^{\oplus 3}$ & $\mathbb{Z}_{2}^{\oplus 2}$ & $\mathbb{Z}_{2}^{\oplus 2}$ & $\mathbb{Z}_{2}$ & 0 \\
\hline $\mathrm{p} 6 \mathrm{~m}$ & $D_{6}$ & $\phi_{2}$ & $\mathbb{Z}_{2}^{\oplus 3}$ & $\mathbb{Z}_{2}^{\oplus 2}$ & $\mathbb{Z}_{2}^{\oplus 2}$ & $\mathbb{Z}_{2}$ & 0 \\
\hline
\end{tabular}

Figure 4. The list of $H_{P}^{3}\left(T^{2} ; \mathbb{Z}_{\phi}\right)$ and its subgroups $F^{p}=F^{p} H_{P}^{3}\left(T^{2} ; \mathbb{Z}_{\phi}\right)$. The $E_{\infty}$-term of the LeraySerre spectral sequence is related to these subgroups by $E_{\infty}^{p, 3-p} \cong F^{p} / F^{p+1}$.

It should be noticed that the action of the point group $P$ on the torus relevant to an application of the Freed-Moore $K$-theory to condensed matter physics is the one modified by 
a non-trivial homomorphism $\phi: P \rightarrow \mathbb{Z}_{2}$. Some of such modified actions differ from those given by the inclusion $P \subset \mathrm{O}(2)$, and hence are not covered in Theorem 1.5. The modified actions should be understood in the context of the so-called magnetic space groups (or colour symmetry groups [25]), and the cohomology as well as the $K$-theory equivariant under the groups deserve to be subjects of a future work.

One may notice that there are more twists for the Freed-Moore $K$-theory in comparison with the twists for equivariant $K$-theory. At present, we lack such an understanding of twists as in Corollary $1.2(\mathrm{c})$ in relation with the nonsymmorphic nature of space groups.

The method for computing $H_{P}^{3}\left(T^{2} ; \mathbb{Z}_{\phi}\right)$ and its filtration is similar to the one computing $H_{P}^{3}\left(T^{2} ; \mathbb{Z}\right)$. In the computation, the cohomology $H_{P}^{n}\left(T^{2} ; \mathbb{Z}_{\phi}\right)$ for $n \leq 2$ is also determined, as summarized below:

Theorem 1.6. Let $P$ be the point group of one of the 2-dimensional space groups $S$, acting on $T^{2}=\mathbb{R}^{2} / \Pi$ via $P \subset \mathrm{O}(2)$. For $n \leq 3$, the $P$-equivariant cohomology $H_{P}^{n}\left(T^{2} ; \mathbb{Z}_{\phi}\right)$ with coefficients in the local system $\mathbb{Z}_{\phi}$ induced from a non-trivial homomorphism $\phi: P \rightarrow \mathbb{Z}_{2}$ is as in Fig. 5 .

\begin{tabular}{|c|c|c||c|c|c|c|}
\hline Space group $S$ & $P$ & $\phi$ & $H_{P}^{0}\left(T^{2}\right)$ & $H_{P}^{1}\left(T^{2}\right)$ & $H_{P}^{2}\left(T^{2}\right)$ & $H_{P}^{3}\left(T^{2}\right)$ \\
\hline $\mathrm{p} 2$ & $\mathbb{Z}_{2}$ & $\phi_{1}$ & 0 & $\mathbb{Z}_{2} \oplus \mathbb{Z}^{\oplus 2}$ & 0 & $\mathbb{Z}_{2}^{\oplus 4}$ \\
\hline $\mathrm{p} 4$ & $\mathbb{Z}_{4}$ & $\phi_{1}$ & 0 & $\mathbb{Z}_{2}$ & $\mathbb{Z}_{2}$ & $\mathbb{Z}_{2}^{\oplus 2}$ \\
\hline $\mathrm{p} 6$ & $\mathbb{Z}_{6}$ & $\phi_{1}$ & 0 & $\mathbb{Z}_{2}$ & $\mathbb{Z}_{3}$ & $\mathbb{Z}_{2}^{\oplus 2}$ \\
\hline $\mathrm{pm} / \mathrm{pg}$ & $D_{1}$ & $\phi_{0}$ & 0 & $\mathbb{Z}_{2} \oplus \mathbb{Z}$ & $\mathbb{Z}_{2} \oplus \mathbb{Z}$ & $\mathbb{Z}_{2}^{\oplus 2}$ \\
\hline $\mathrm{cm}$ & $D_{1}$ & $\phi_{0}$ & 0 & $\mathbb{Z}_{2} \oplus \mathbb{Z}$ & $\mathbb{Z}$ & $\mathbb{Z}_{2}$ \\
\hline $\mathrm{pmm} / \mathrm{pmg} / \mathrm{pgg}$ & $D_{2}$ & $\phi_{0}$ & 0 & $\mathbb{Z}_{2}$ & $\mathbb{Z}_{2}^{\oplus 3} \oplus \mathbb{Z}$ & $\mathbb{Z}_{2}^{\oplus 4}$ \\
\hline $\mathrm{pmm} / \mathrm{pmg} / \mathrm{pgg}$ & $D_{2}$ & $\phi_{1}, \phi_{2}$ & 0 & $\mathbb{Z}_{2} \oplus \mathbb{Z}$ & $\mathbb{Z}_{2}^{\oplus 2}$ & $\mathbb{Z}_{2}^{\oplus 6}$ \\
\hline $\mathrm{cmm}$ & $D_{2}$ & $\phi_{0}$ & 0 & $\mathbb{Z}_{2}$ & $\mathbb{Z}_{2}^{\oplus 2} \oplus \mathbb{Z}$ & $\mathbb{Z}_{2}^{\oplus 2}$ \\
\hline $\mathrm{cmm}$ & $D_{2}$ & $\phi_{1}, \phi_{2}$ & 0 & $\mathbb{Z}_{2} \oplus \mathbb{Z}$ & $\mathbb{Z}_{2}$ & $\mathbb{Z}_{2}^{\oplus 4}$ \\
\hline $\mathrm{p} 3 \mathrm{~m} 1$ & $D_{3}$ & $\phi_{0}$ & 0 & $\mathbb{Z}_{2}$ & $\mathbb{Z}_{3}^{\oplus 2} \oplus \mathbb{Z}$ & $\mathbb{Z}_{2}$ \\
\hline $\mathrm{p} 31 \mathrm{~m}$ & $D_{3}$ & $\phi_{0}$ & 0 & $\mathbb{Z}_{2}$ & $\mathbb{Z}_{3} \oplus \mathbb{Z}$ & $\mathbb{Z}_{2}$ \\
\hline $\mathrm{p} 4 \mathrm{~m} / \mathrm{p} 4 \mathrm{~g}$ & $D_{4}$ & $\phi_{0}$ & 0 & $\mathbb{Z}_{2}$ & $\mathbb{Z}_{4} \oplus \mathbb{Z}_{2} \oplus \mathbb{Z}$ & $\mathbb{Z}_{2}^{\oplus 3}$ \\
\hline $\mathrm{p} 4 \mathrm{~m} / \mathrm{p} 4 \mathrm{~g}$ & $D_{4}$ & $\phi_{1}, \phi_{2}$ & 0 & $\mathbb{Z}_{2}$ & $\mathbb{Z}_{2}^{\oplus 2}$ & $\mathbb{Z}_{2}^{\oplus 4}$ \\
\hline $\mathrm{p} 6 \mathrm{~m}$ & $D_{6}$ & $\phi_{0}$ & 0 & $\mathbb{Z}_{2}$ & $\mathbb{Z}_{6} \oplus \mathbb{Z}$ & $\mathbb{Z}_{2}^{\oplus 2}$ \\
\hline $\mathrm{p} 6 \mathrm{~m}$ & $D_{6}$ & $\phi_{1}$ & 0 & $\mathbb{Z}_{2}$ & $\mathbb{Z}_{2} \oplus \mathbb{Z} 3$ & $\mathbb{Z}_{2}^{\oplus 3}$ \\
\hline $\mathrm{p} 6 \mathrm{~m}$ & $D_{6}$ & $\phi_{2}$ & 0 & $\mathbb{Z}_{2}$ & $\mathbb{Z}_{2}$ & $\mathbb{Z}_{2}^{\oplus 3}$ \\
\hline
\end{tabular}

Figure 5. The list of equivariant cohomology with local coefficients.

Finally, we make comments about the generalizations. To compute cohomology groups of the higher-dimensional tori which are equivariant under space groups, we can in principle apply the three methods in this paper. The first and second methods would be generalized without difficulty. The third method will however get more difficult, because we need a $P$-CW decomposition of a higher-dimensional torus, which becomes more complicated than decompositions in the 2-dimensional case. As is suggested by Corollary 1.4, there are local systems for the Borel equivariant cohomology other than $\mathbb{Z}_{\phi}$ associated to a homomorphism $\phi: P \rightarrow \mathbb{Z}_{2}$. For the cohomology with such a local system, the notion of reduced cohomology does not make sense. 
This prevents us from using the second method based on the equivariant stable splitting of the torus, forcing us to use a $P$-CW decomposition.

The outline of this paper is as follows: In Section 2, we explain how a certain quantum system leads to a twist and defines a twisted $K$-class, mainly based on a formulation in [8]. At the end of this section, a summary of relationship among some natural actions of point groups on tori is included. In Section 3, we review the Leray-Serre spectral sequence for Borel equivariant cohomology and the notion of twists for equivariant $K$-theory. The geometric interpretation of the filtration of the degree 3 equivariant cohomology is also provided here, after a general property of the spectral sequence is established. Then, in Section 4, we prove Theorems 1.1 and 1.3. To keep readability of this paper, we provide the detail of computations only in the cases $\mathrm{p} 2, \mathrm{p} 4 \mathrm{~m} / \mathrm{p} 4 \mathrm{~g}$ and $\mathrm{p} 6 \mathrm{~m}$. (The detail of the other cases can be found in old versions of arXiv:1509.09194.) Section 5 concerns the equivariant cohomology with the twisted coefficient $\mathbb{Z}_{\phi}$. We state direct generalizations of some results in the untwisted case, and then prove Theorems 1.5 and 1.6. To keep readability again, we give the details of the computation only in the case of $\mathrm{p} 6 \mathrm{~m}$ with $\phi_{2}$. Finally, for convenience, the point group actions of 2-dimensional space groups are listed in Appendix.

Throughout, familiarity with basic algebraic topology [4, 11] will be supposed.

\section{From quantum systems to twisted $K$-theory}

We here illustrate how twisted equivariant $K$-theory arises from a quantum system with symmetry, mainly based on a formulation in [8]. (We refer the reader to [29] for a $C^{*}$-algebraic approach.)

\section{$2.1 \quad$ Setting}

Let us consider the following mathematical setting:

- A lattice $\Pi \subset \Pi \otimes_{\mathbb{Z}} \mathbb{R}=\mathbb{R}^{d}$ of rank $d$.

- A subgroup $S$ of the Euclidean group $\mathbb{R}^{d} \rtimes \mathrm{O}(d)$ of $\mathbb{R}^{d}$ which is an extension of a finite group $P \subset \mathrm{O}(d)$ by $\Pi$ :

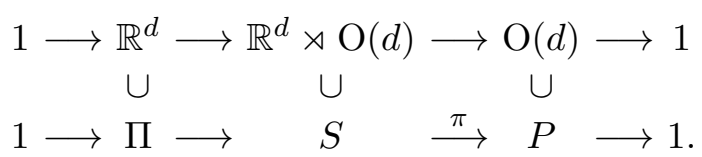

- A unitary representation $U: P \rightarrow U(V)$ on a finite-dimensional Hermitian vector space $V$.

The group $S$ is nothing but a $d$-dimensional space group, and $P$ is called the point group of $S$. When $S$ is the semi-direct product of $P$ and $\Pi$, it is called symmorphic, otherwise nonsymmorphic.

Based on the mathematical setting above, we can introduce a quantum system on $\mathbb{R}^{d}$ which has $S$ as its symmetry and $V$ as its internal freedom:

- The 'quantum Hilbert space' consisting of 'wave functions' is the $L^{2}$-space $L^{2}\left(\mathbb{R}^{d}, V\right)$, on which $g \in S$ acts by $\psi(x) \mapsto(\rho(g) \psi)(x)=U(\pi(g)) \psi\left(g^{-1} x\right)$.

- The 'Hamiltonian' is a self-adjoint operator $H$ on $L^{2}\left(\mathbb{R}^{d}, V\right)$ invariant under the $S$-action: $H \circ \rho(g)=\rho(g) \circ H$. A typical form of $H$ is $H=\Delta+\Phi$, where $\Delta=\sum \partial^{2} / \partial x_{i}^{2}$ is the Laplacian and $\Phi: \mathbb{R}^{d} \rightarrow \operatorname{End}(V)$ is a potential term. 


\subsection{Bloch transformation}

Even if the Hamiltonian $H$ is invariant under the translation of $\Pi$, a solution $\psi$ to the 'timeindependent Schrödinger equation' $H \psi=E \psi$ with $E \in \mathbb{R}$ is not necessarily $S$-invariant. The so-called 'Bloch transformation' allows us to deal with such a situation.

Let $\hat{\Pi}=\operatorname{Hom}(\Pi, U(1))$ denote the Pontryagin dual of the lattice $\Pi$, which is often called the 'Brillouin torus' in condensed matter physics. We define the space $L_{\Pi}^{2}\left(\hat{\Pi} \times \mathbb{R}^{d}, V\right)$ by

$$
L_{\Pi}^{2}\left(\hat{\Pi} \times \mathbb{R}^{d}, V\right)=\left\{\hat{\psi} \in L^{2}\left(\hat{\Pi} \times \mathbb{R}^{d}, V\right) \mid \hat{\psi}(\hat{k}, x+m)=\hat{k}(m) \hat{\psi}(\hat{k}, x)(m \in \Pi)\right\} .
$$

We also define transformations $\hat{\mathcal{B}}$ and $\mathcal{B}$, inverse to each other:

$$
\begin{array}{ll}
\hat{\mathcal{B}}: & L^{2}\left(\mathbb{R}^{d}, V\right) \longrightarrow L_{\Pi}^{2}\left(\hat{\Pi} \times \mathbb{R}^{d}, V\right), \quad(\hat{\mathcal{B}} \psi)(\hat{k}, x)=\sum_{n \in \Pi} \hat{k}(n)^{-1} \psi(x+n), \\
\mathcal{B}: & L_{\Pi}^{2}\left(\hat{\Pi} \times \mathbb{R}^{d}, V\right) \longrightarrow L^{2}\left(\mathbb{R}^{d}, V\right), \quad(\mathcal{B} \hat{\psi})(x)=\int_{\hat{k} \in \hat{\Pi}} \hat{\psi}(\hat{k}, x) d \hat{k} .
\end{array}
$$

As is described in [8], the space $L_{\Pi}^{2}\left(\hat{\Pi} \times \mathbb{R}^{d}, V\right)$ can be identified with the space $L^{2}(\hat{\Pi}, \mathcal{E} \otimes V)$ of $L^{2}$-sections of a vector bundle $\mathcal{E} \otimes V \rightarrow \hat{\Pi}$. The infinite-dimensional vector bundle $\mathcal{E} \rightarrow \hat{\Pi}$ is given by

$$
\mathcal{E}=\bigcup_{\hat{k} \in \hat{\Pi}} L^{2}\left(\mathbb{R}^{d} / \Pi,\left.\mathcal{L}\right|_{\{\hat{k}\} \times \mathbb{R}^{d} / \Pi}\right)
$$

where $\mathcal{L} \rightarrow \hat{\Pi} \times \mathbb{R}^{d} / \Pi$ is the Poincaré line bundle, the quotient of the product line bundle $\hat{\Pi} \times \mathbb{R}^{d} \times \mathbb{C} \rightarrow \hat{\Pi} \times \mathbb{R}^{d}$ by the following $\Pi$-action

$$
\Pi \times\left(\hat{\Pi} \times \mathbb{R}^{d} \times \mathbb{C}\right) \longrightarrow \hat{\Pi} \times \mathbb{R}^{d} \times \mathbb{C}, \quad(m, \hat{k}, x, z) \mapsto(\hat{k}, x+m, \hat{k}(m) z) .
$$

In summary, we get an identification of $L^{2}$-spaces

$$
L^{2}\left(\mathbb{R}^{d}, V\right) \cong L_{\Pi}^{2}\left(\hat{\Pi} \times \mathbb{R}^{d}, V\right) \cong L^{2}(\hat{\Pi}, \mathcal{E} \otimes V) .
$$

The Hamiltonian $H$ on $L^{2}\left(\mathbb{R}^{d}, V\right)$ then induces an operator $\hat{H}$ on $L_{\Pi}^{2}\left(\hat{\Pi} \times \mathbb{R}^{d}, V\right) \cong L^{2}(\hat{\Pi}, \mathcal{E} \otimes$ $V)$ by $\hat{H} \circ \hat{\mathcal{B}}=\hat{\mathcal{B}} \circ H$. If, for instance, $H$ is of the form $H=\Delta+\Phi$, then $\hat{H}$ preserves the fiber of $\mathcal{E} \otimes V$. Generally, this is a consequence of the translation invariance of the Hamiltonian. When the present quantum system is supposed to be an 'insulator', a finite number of discrete spectra of $\hat{H}(\hat{k})$ would be confined to a compact region in $\mathbb{R}$ as $\hat{k} \in \hat{\Pi}$ varies. Then the corresponding eigenfunctions form a finite rank subbundle $E \subset \mathcal{E} \otimes V$, called the 'Bloch bundle'. The $K$-class of this vector bundle $E \rightarrow \hat{\Pi}$ is regarded as an invariant of the quantum system under study.

\subsection{Nonsymmorphic group and twisted $K$-theory}

We now take the symmetry into account. From the extension $1 \rightarrow \Pi \rightarrow S \stackrel{\pi}{\rightarrow} P \rightarrow 1$, we can associate a twisted $P$-equivariant vector bundle on $\hat{\Pi}$ to the $S$-module $L^{2}\left(\mathbb{R}^{d}, V\right)$. This is a version of the so-called 'Mackey machine'.

Recall that the Euclidean group $\mathbb{R}^{d} \rtimes \mathrm{O}(d)$ is the semi-direct product of the orthogonal group $\mathrm{O}(d)$ and the group of translations $\mathbb{R}^{d}$. Hence a collection of representatives $\left\{s_{p}\right\}_{p \in P}$ of $p \in P \cong S / \Pi$ in $S$ is expressed as $s_{p}=\left(a_{p}, p\right) \in \mathbb{R}^{d} \rtimes \mathrm{O}(d)$ by means of a map $a: P \rightarrow \mathbb{R}^{d}$. For $p_{1}, p_{2} \in P$ we put

$$
\nu\left(p_{1}, p_{2}\right)=a_{p_{1}}+p_{1} a_{p_{2}}-a_{p_{1} p_{2}} .
$$


Since $\Pi \subset S$ is normal, the action of $P \subset \mathrm{O}(d)$ on $\mathbb{R}^{d}$ preserves $\Pi \subset \mathbb{R}^{d}$. Then we have $\nu\left(p_{1}, p_{2}\right) \in \Pi$, and $\nu: P \times P \rightarrow \Pi$ is a group 2-cocycle of $P$ with values in $\Pi$ regarded as a left $P$-module through the action $m \mapsto p m$ of $p \in P$ on $m \in \Pi$. This group 2-cocycle measures the failure for $S$ to be symmorphic.

By means of the $S$-action $\rho$ on $L^{2}\left(\mathbb{R}^{d}, V\right)$, we define an 'action' of $p \in P$ by

$$
\rho(p): L^{2}\left(\mathbb{R}^{d}, V\right) \longrightarrow L^{2}\left(\mathbb{R}^{d}, V\right), \quad \rho(p)=\rho\left(\left(a_{p}, p\right)\right),
$$

whose explicit formula for $\psi \in L^{2}\left(\mathbb{R}^{d}, V\right)$ is given by $(\rho(p) \psi)(x)=U(p) \psi\left(p^{-1} x+a_{p^{-1}}\right)$. The Bloch transformation then induces the following 'action' of $P$,

$$
\hat{\rho}(p): L_{\Pi}^{2}\left(\hat{\Pi} \times \mathbb{R}^{d}, V\right) \rightarrow L_{\Pi}^{2}\left(\hat{\Pi} \times \mathbb{R}^{d}, V\right), \quad \hat{\rho}(p) \circ \hat{\mathcal{B}}=\hat{\mathcal{B}} \circ \rho(p),
$$

whose explicit formula for $\hat{\psi} \in L_{\Pi}^{2}\left(\hat{\Pi} \times \mathbb{R}^{d}, V\right)$ is $(\hat{\rho}(p) \hat{\psi})(\hat{k}, x)=U(p) \hat{\psi}\left(p^{-1} \hat{k}, p^{-1} x+a_{p^{-1}}\right)$. Here the left $P$-action on $\hat{\Pi}$ is defined by $(p \hat{k})(m)=\hat{k}\left(p^{-1} m\right)$, where $p \in P$ acts on $m \in \Pi$ through the inclusion $P \subset \mathrm{O}(d)$ and the left action of $\mathrm{O}(d)$ on $\mathbb{R}^{d}$. Notice that $\rho$ and $\hat{\rho}$ can be honest actions of $P$ in the case of symmorphic $S$, but not in the case of nonsymmorphic $S$, for the usual composition rule is violated:

$$
\left(\hat{\rho}\left(p_{1}\right)\left(\hat{\rho}\left(p_{2}\right) \hat{\psi}\right)\right)(\hat{k}, \xi)=\left(p_{2}^{-1} p_{1}^{-1} \hat{k}\right)\left(\nu\left(p_{2}^{-1}, p_{1}^{-1}\right)\right)\left(\hat{\rho}\left(p_{1} p_{2}\right) \hat{\psi}\right)(\hat{k}, \xi) .
$$

To interpret the 'action' $\hat{\rho}(p)$ in terms of the vector bundle $\mathcal{E} \otimes V$ through $L_{\Pi}^{2}\left(\hat{\Pi} \times \mathbb{R}^{d}, V\right) \cong$ $L^{2}(\hat{\Pi}, \mathcal{E} \otimes V)$, recall that the fiber of $\mathcal{E} \otimes V$ at $\hat{k} \in \hat{\Pi}$ is

$$
\left.\mathcal{E}\right|_{\hat{k}} \otimes V=L^{2}\left(\mathbb{R}^{d} / \Pi,\left.\mathcal{L}\right|_{\{\hat{k}\} \times \mathbb{R}^{d} / \Pi} \otimes V\right),
$$

and $\hat{\psi} \in L_{\Pi}^{2}\left(\hat{\Pi} \times \mathbb{R}^{d}, V\right)$ corresponds to the following section $\Psi \in L^{2}(\hat{\Pi}, \mathcal{E} \otimes V)$ :

$$
\Psi(\hat{k}): \mathbb{R}^{d} /\left.\Pi \longrightarrow \mathcal{L}\right|_{\{\hat{k}\} \times \mathbb{R}^{d} / \Pi} \otimes V, \quad x \mapsto[\hat{k}, x, \hat{\psi}(\hat{k}, x)] .
$$

Define for $p \in P$ and $\hat{k} \in \hat{\Pi}$ a linear map

$$
\rho_{\mathcal{E} \otimes V}(p ; \hat{k}):\left.\left.\mathcal{E}\right|_{\hat{k}} \otimes V \longrightarrow \mathcal{E}\right|_{p \hat{k}} \otimes V
$$

by the assignment of the sections

$$
\rho_{\mathcal{E} \otimes V}(p ; \hat{k})([x \mapsto[\hat{k}, x, \hat{\psi}(\hat{k}, x)]])=\left[x \mapsto\left[p \hat{k}, x, U(p) \hat{\psi}\left(\hat{k}, p^{-1} x+a_{p^{-1}}\right)\right]\right]
$$

These maps constitute a vector bundle map $\rho_{\mathcal{E} \otimes V}(p): \mathcal{E} \otimes V \rightarrow \mathcal{E} \otimes V$ covering the action $\hat{k} \mapsto p \hat{k}$ on $\hat{\Pi}$

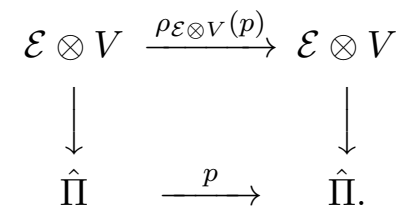

This is a $\tau$-twisted $P$-action, in the sense that the formula

$$
\rho_{\mathcal{E} \otimes V}\left(p_{1} ; p_{2} \hat{k}\right) \rho_{\mathcal{E} \otimes V}\left(p_{2} ; \hat{k}\right) \xi=\tau\left(p_{1}, p_{2} ; \hat{k}\right) \rho_{\mathcal{E} \otimes V}\left(p_{1} p_{2} ; \hat{k}\right) \xi
$$

holds for $p_{1}, p_{2} \in P, \hat{k} \in \hat{\Pi}$ and $\left.\xi \in \mathcal{E}\right|_{\hat{k}} \otimes V$. Here $\tau: P \times P \times \hat{\Pi} \rightarrow U(1)$ is defined by

$$
\tau\left(p_{1}, p_{2} ; \hat{k}\right)=\hat{k}\left(\nu\left(p_{2}^{-1}, p_{1}^{-1}\right)\right)
$$


and is regarded as a group 2-cocycle of $P$ with its coefficients in the group $C(\hat{\Pi}, U(1))$ of $U(1)$ valued functions on $\hat{\Pi}$ thought of as a right $P$-module through the pull-back under the left action $\hat{k} \mapsto p \hat{k}$ of $p \in P$ on $\hat{k} \in \hat{\Pi}$. The map $\rho_{\mathcal{E} \otimes V}(p)$ on the vector bundle induces the transformation on the sections

$$
\rho_{\mathcal{E} \otimes V}(p): L^{2}(\hat{\Pi}, \mathcal{E} \otimes V) \longrightarrow L^{2}(\hat{\Pi}, \mathcal{E} \otimes V)
$$

by $\left(\rho_{\mathcal{E} \otimes V}(p) \Psi\right)(\hat{k})=\rho_{\mathcal{E} \otimes V}\left(p ; p^{-1} \hat{k}\right) \Psi\left(p^{-1} \hat{k}\right)$. One can verify that: if $\Psi \in L^{2}(\hat{\Pi}, \mathcal{E} \otimes V)$ corresponds to $\hat{\psi} \in L_{\Pi}^{2}\left(\hat{\Pi} \times \mathbb{R}^{d}, V\right)$, then $\rho_{\mathcal{E} \otimes V}(p) \Psi$ corresponds to $\hat{\rho}(p) \hat{\psi}$. Hence the 'action' $\hat{\rho}(p)$ on $L_{\Pi}^{2}\left(\hat{\Pi} \times \mathbb{R}^{d}, V\right) \cong L^{2}(\hat{\Pi}, \mathcal{E} \otimes V)$ agrees with the one induced from the $\tau$-twisted $P$-action on $\mathcal{E} \otimes V$.

Now, under the assumption that $\hat{H}$ describes an insulator, the Bloch bundle $E \subset \mathcal{E} \otimes V$ inherits a $\tau$-twisted $P$-action from $\mathcal{E} \otimes V$. This is a consequence of the invariance of the Hamiltonian under the space group action. Therefore the Bloch bundle, being a $\tau$-twisted $P$-equivariant vector bundle of finite rank, defines a class in the $\tau$-twisted $P$-equivariant $K$-theory $K_{P}^{\tau+0}(\hat{\Pi})$, which is regarded as an invariant of the insulating system under study.

As is obvious from the construction, we can apply the construction of the group 2-cocycle $\tau$ to symmorphic space groups. However, in the symmorphic case, the cocycle $\nu$ and hence $\tau$ can be trivialized.

So far a linear representation of $P$ on $V$ is considered. We can relax this representation to be a projective representation of $P$ with its group 2-cocycle $\omega: P \times P \rightarrow U(1)$. In this case, the resulting Bloch bundle defines a class in the twisted equivariant $K$-theory $K_{P}^{\tau+\omega+0}(\hat{\Pi})$.

Remark 2.1. The phase factor in the composition rule of $\hat{\rho}$,

$$
\tau_{R}\left(\hat{k} ; p_{1}, p_{2}\right)=\left(p_{2}^{-1} p_{1}^{-1} \hat{k}\right)\left(\nu\left(p_{2}^{-1}, p_{1}^{-1}\right)\right)=\hat{k}\left(p_{1} p_{2} \nu\left(p_{2}^{-1}, p_{1}^{-1}\right)\right)
$$

defines a group 2-cocycle of $P$ with coefficients in $C(\hat{\Pi}, U(1))$, when regarded as a left $P$-module by the right action $\hat{k} \mapsto \hat{k} p=p^{-1} \hat{k}$ of $p \in P$ on $\hat{k} \in \hat{\Pi}$. The 2-cocycles $\tau$ and $\tau_{R}$ are related by $\tau_{R}\left(\hat{k} ; p_{1}, p_{2}\right)=\tau\left(p_{1}, p_{2} ;\left(p_{1} p_{2}\right)^{-1} \hat{k}\right)$. This extends to a cochain bijection of group cochains with coefficients in the left/right $P$-modules $C(\hat{\Pi}, U(1))$. Thus, $\tau$ and $\tau_{R}$ have cohomologically the same information. We also remark that $\tau$ and $\tau_{R}$ are respectively cohomologous to the following 2-cocycles:

$$
\tau^{\prime}\left(p_{1}, p_{2} ; \hat{k}\right)=\left(p_{1} p_{2} \hat{k}\right)\left(\nu\left(p_{1}, p_{2}\right)\right)^{-1}, \quad \tau_{R}^{\prime}\left(\hat{k} ; p_{1}, p_{2}\right)=\hat{k}\left(\nu\left(p_{1}, p_{2}\right)\right)^{-1} .
$$

Remark 2.2. Given a homomorphism $c: P \rightarrow \mathbb{Z}_{2}$, we can impose that the Hamiltonian $H$ and the symmetry $\rho(g)$ with $g \in S$ are graded commutative, $H \circ \rho(g)=c(\pi(g)) \rho(g) \circ H$. Then the quantum system with symmetry in question leads to an element of the twisted equivariant $K$-theory $K_{P}^{\tau+c+0}(\hat{\Pi})$, where the (ungraded) twist $\tau$ is now graded by $c \in H_{P}^{1}\left(\hat{\Pi} ; \mathbb{Z}_{2}\right)$. It should be noticed that the construction of the element uses Karoubi's formulation of $K$-theory [13] and requires a reference quantum system. These points of discussion, which will not be detailed in this paper, are implicit in the absence of the graded twist.

Remark 2.3. A group 2-cocycle $\tau$ can be thought of as the cocycle for a projective representation. Besides the argument in this section, there are other arguments which derive projective representations from quantum systems with symmetry (for example $[15,16]$ ).

\subsection{Actions of the point group on the torus}

To close Section 2, we compare some natural actions of the point group on the torus: Let $S$ be a $d$-dimensional space group, $\Pi$ its lattice, and $P$ its point group. 
(A) By the inclusion $P \subset \mathrm{O}(d)$ and the standard left action of $\mathrm{O}(d)$ on $\mathbb{R}^{d}$, the point group $P$ acts on $\mathbb{R}^{d}$, preserving $\Pi \subset \mathbb{R}^{d}$. Hence the left action of $P$ on $\mathbb{R}^{d}$ descends to give a left action of $P$ on the torus $\mathbb{R}^{d} / \Pi$.

(B) By the action (A), the point group $P$ acts on the Pontryagin dual $\hat{\Pi}=\operatorname{Hom}(\Pi, U(1))$ of $\Pi$ from the left: For $p \in P$ and $\hat{k} \in \hat{\Pi}$, we define $p \hat{k} \in \hat{\Pi}$ by $(p \hat{k})(m)=\hat{k}\left(p^{-1} m\right)$ for all $m \in \Pi$.

(C) By the inclusion $S \subset \mathbb{R}^{d} \rtimes \mathrm{O}(d)$ and the standard left action of $\mathbb{R}^{d} \rtimes \mathrm{O}(d)$ on $\mathbb{R}^{d}$, the space group $S$ acts on $\mathbb{R}^{d}$. The subgroup $\Pi \subset S$ preserves $\Pi \subset \mathbb{R}^{d}$, so that the point group $P \cong S / \Pi$ acts on $\mathbb{R}^{d} / \Pi$.

The action (A) is what we consider in our main results, and the action (B) is relevant to quantum systems as reviewed in this section.

On the one hand, the actions $(\mathrm{A})$ and $(\mathrm{B})$ clearly fix $0 \in \mathbb{R}^{d} / \Pi$ and $0 \in \hat{\Pi}$, respectively, where we regard $\hat{\Pi}$ as $\operatorname{Hom}(\Pi, \mathbb{R} / \mathbb{Z})$ via $\mathbb{R} / \mathbb{Z} \cong U(1)$ and $0 \in \hat{\Pi}$ stands for the trivial homomorphism. On the other hand, if $\left(a_{p}, p\right) \in \mathbb{R}^{d} \rtimes \mathrm{O}(d)$ is a lift of $p \in P \subset \mathrm{O}(d)$, then the action of $p \in P$ on $k \in \mathbb{R}^{d} / \Pi$ in $(\mathrm{C})$ admits the description $k \mapsto p k+a_{p}$. If $S$ is symmorphic, then we can choose $a_{p}$ to be in $\Pi$. In this case, the actions (A) and (C) are equivalent. However, if $S$ is nonsymmorphic, then $a_{p}$ cannot be in $\Pi$. Thus, in this case, the action of $p \in P$ does not fix any point on $\mathbb{R}^{d} / \Pi$, so that the actions $(\mathrm{A})$ and $(\mathrm{C})$ are not equivalent. For example, in the case of $\mathrm{pg}$, the action of $P=\mathbb{Z}_{2}$ on the 2-dimensional torus is free, and its quotient is the Klein bottle.

To compare the actions (A) and (B), we need to identify $\mathbb{R}^{d} / \Pi$ with $\hat{\Pi}=\operatorname{Hom}(\Pi, \mathbb{R} / \mathbb{Z})$, which are topologically $d$-dimensional tori. In general, such an identification may not be unique. A way to implement the identification is to choose a basis $\left\{v_{j}\right\}$ of the lattice $\Pi \cong \mathbb{Z}^{d}$. This choice induces the following identifications of tori inverse to each other:

$$
\begin{aligned}
& \hat{\Pi} \longrightarrow \mathbb{R}^{d} / \Pi, \quad \hat{k} \mapsto \sum_{j} \hat{k}\left(v_{j}\right) v_{j}, \\
& \mathbb{R}^{d} / \Pi \longrightarrow \hat{\Pi}, \quad \sum_{j} k_{j} v_{j} \mapsto\left[\sum_{j} m_{j} v_{j} \mapsto \sum_{j} m_{j} k_{j}\right] .
\end{aligned}
$$

With this identification of tori, the left $P$-action on $\hat{\Pi}$ in (B) induces a right $P$-action on $\mathbb{R}^{d} / \Pi$. Considering the action of $p^{-1}$ instead of $p$, we finally get a left action of $P$ on $\mathbb{R}^{d} / \Pi$, induced from (B) and the identification $\hat{\Pi} \cong \mathbb{R}^{d} / \Pi$. In general, this left action of $P$ on $\mathbb{R}^{d} / \Pi$ induced from (B) is not equivalent to the action (A). In the 2-dimensional case, their relationship is as follows:

Lemma 2.4. Let $S$ be a 2-dimensional space group, $P_{S}$ its point group, $\Pi_{S}$ its lattice, and $\hat{\Pi}_{S}$ the Pontryagin dual of $\Pi_{S}$.

(a) We choose a basis $\left\{v_{j}\right\}$ of $\Pi_{S}$ to identify $\hat{\Pi}_{S}$ with $\mathbb{R}^{2} / \Pi_{S}$, and let the action in $(B)$ induce an action of $P_{S}$ on $\mathbb{R}^{2} / \Pi_{S}$. Then, up to equivalence, this action is independent of the choice of the basis.

(b) If $S$ is not $p 3 m 1$ or $p 31 m$, then the action of $P_{S}$ on $\mathbb{R}^{2} / \Pi_{S}$ induced from $(B)$ is equivalent to the action of $P_{S}$ on $\mathbb{R}^{2} / \Pi_{S}$ in $(A)$.

(c) If $S$ is $p 3 m 1$ (respectively $p 31 m$ ), then the action of $P_{S}$ on $\mathbb{R}^{2} / \Pi_{S}$ induced from $(B)$ is equivalent to the action of $P_{S^{\prime}}$ on $\mathbb{R}^{2} / \Pi_{S^{\prime}}$ in $(A)$, where $S^{\prime}$ is p31m (respectively $p 3 m 1$ ).

We remark that the space groups $\mathrm{p} 3 \mathrm{~m} 1$ and $\mathrm{p} 31 \mathrm{~m}$ share the same lattice and the same point group, as can be seen in Appendix A. Hence we have $P_{S}=P_{S^{\prime}}$ and $\Pi_{S}=\Pi_{S^{\prime}}$ in the third item in the lemma above. 
Proof. Considering the action $(A)$, we define $\psi(p)_{\ell j} \in \mathbb{Z}$ by $p v_{j}=\sum_{\ell} \psi(p)_{\ell_{j}} v_{\ell}$, and a homomorphism $\psi: P_{S} \rightarrow \mathrm{GL}(2, \mathbb{Z})$ by $\psi(p)=\left(\psi(p)_{\ell j}\right)$. Since $P_{S}$ is the point group of a 2-dimensional space group $S$, the homomorphism $\psi$ is injective and its image $\psi\left(P_{S}\right)$ is a finite subgroup of $\mathrm{GL}(2, \mathbb{Z})$. Let $S^{\prime}$ be another 2-dimensional space group with its point group $P^{\prime}$. Choosing a basis of its lattice $\Pi^{\prime}$, we similarly get from the action (A) a homomorphism $\psi^{\prime}: P_{S^{\prime}} \rightarrow \mathrm{GL}(2, \mathbb{Z})$. If the images $\psi\left(P_{S}\right)$ and $\psi^{\prime}\left(P_{S^{\prime}}\right)$ are conjugate to each other in $\operatorname{GL}(2, \mathbb{Z})$, then the actions of $P_{S}$ and $P_{S^{\prime}}$ in (A) are equivalent. The action of $P_{S}$ on $\mathbb{R}^{2} / \Pi_{S}$ induced from (B) also yields an associated homomorphism $P_{S} \rightarrow \mathrm{GL}(2, \mathbb{Z})$. This homomorphism turns out to be the transpose inverse ${ }^{t} \psi^{-1}: P_{S} \rightarrow \mathrm{GL}(2, \mathbb{Z})$, which is again injective and defines a finite subgroup ${ }^{t} \psi^{-1}\left(P_{S}\right) \subset \mathrm{GL}(2, \mathbb{Z})$. If we alter the basis $\left\{v_{j}\right\}$, then ${ }^{t} \psi^{-1}$ changes by a conjugation of a matrix in $\operatorname{GL}(2, \mathbb{Z})$. Thus, up to conjugations, the image ${ }^{t} \psi^{-1}\left(P_{S}\right) \subset \mathrm{GL}(2, \mathbb{Z})$ is independent of the choice of $\left\{v_{j}\right\}$, showing (a). Now we can directly verify (b) and (c), by computing the homomorphism $\psi$ based on the explicit basis in Appendix, and comparing the images $\psi\left(P_{S}\right)$ and ${ }^{t} \psi^{-1}\left(P_{S}\right)$ in $\operatorname{GL}(2, \mathbb{Z})$.

Another way of identifying $\hat{\Pi}$ with $\mathbb{R}^{d} / \Pi$ is to choose a bilinear form $\langle\rangle:, \Pi \times \Pi \rightarrow \mathbb{Z}$. We assume that this form is non-degenerate in the sense that the matrix $\left(\left\langle v_{i}, v_{j}\right\rangle\right)$ is invertible with respect to any basis $\left\{v_{i}\right\}$ of $\Pi$. A non-degenerate bilinear form induces an identification of the tori as follows

$$
\mathbb{R}^{d} / \Pi \longrightarrow \hat{\Pi}=\operatorname{Hom}(\Pi, \mathbb{R} / \mathbb{Z}), \quad k \mapsto[m \mapsto\langle m, k\rangle] .
$$

If the bilinear form is $P$-invariant in the sense that $\left\langle p m, p m^{\prime}\right\rangle=\left\langle m, m^{\prime}\right\rangle$ for all $m, m^{\prime} \in \Pi$ and $p \in P$, then the action $(\mathrm{A})$ on $\mathbb{R}^{d} / \Pi$ agrees with the action $(\mathrm{B})$ on $\hat{\Pi}$ under the induced identification $\mathbb{R}^{d} / \Pi \cong \hat{\Pi}$. For the 2 -dimensional space groups such that $\Pi$ can be the standard lattice $\mathbb{Z}^{2} \subset \mathbb{R}^{2}$, the standard inner product on $\mathbb{R}^{2}$ restricts to give a $P$-invariant non-degenerate bilinear form. If we choose an orthonormal basis $\left\{v_{j}\right\}$, then the identifications $\mathbb{R}^{d} / \Pi \cong \hat{\Pi}$ given by $\langle$,$\rangle and by \left\{v_{j}\right\}$ are $P$-equivariantly the same.

In Section 4, we will work with the torus $\mathbb{R}^{2} / \Pi$ with the action (A), and the relation to $\hat{\Pi}$ with the action (B) should be understood as above.

\section{The Leray-Serre spectral sequence and twists}

This section gives a geometric interpretation of the filtration of $H_{G}^{3}(X ; \mathbb{Z})$ for the Leray-Serre spectral sequence through types of twists. This is carried out by identifying the Leray-Serre spectral sequence with another natural spectral sequence which computes the Borel equivariant cohomology.

Throughout this section, we assume that $G$ is a finite group acting from the left on a 'reasonable' space $X$, such as a locally contractible, paracompact and regular topological space as in [7], or a $G$-CW complex [19].

\subsection{Spectral sequences}

The Borel equivariant cohomology $H_{G}^{n}(X ; \mathbb{Z})$ is defined to be the (singular) cohomology of the quotient space $E G \times_{G} X$ of $E G \times X$ under the diagonal $G$-action $(\xi, x) \mapsto\left(\xi g, g^{-1} x\right)$, where $E G$ is the total space of the universal $G$-bundle $E G \rightarrow B G$. Associated to the fibration $X \rightarrow E G \times_{G} X \rightarrow B G$ is the Leray-Serre spectral sequence

$$
E_{r}^{p, q} \Longrightarrow H_{G}^{p+q}(X ; \mathbb{Z})
$$

converging to the graded quotient of a filtration

$$
H_{G}^{n}(X ; \mathbb{Z})=F^{0} H_{G}^{n}(X ; \mathbb{Z}) \supset F^{1} H_{G}^{n}(X ; \mathbb{Z}) \supset \cdots \supset F^{n+1} H_{G}^{n}(X ; \mathbb{Z})=0,
$$


that is, $E_{\infty}^{p, q}=F^{p} H_{G}^{p+q}(X ; \mathbb{Z}) / F^{p+1} H_{G}^{p+q}(X ; \mathbb{Z})$. The $E_{2}$-term is given by the group cohomology of $G$

$$
E_{2}^{p, q}=H_{\text {group }}^{p}\left(G ; H^{q}(X ; \mathbb{Z})\right),
$$

where the coefficient $H^{q}(X ; \mathbb{Z})$ is regarded as a right $G$-module by the pull-back action. As a convention of this paper, the group of $p$-cochains with values in a right $G$-module $M$ is denoted by $C_{\text {group }}^{p}(G ; M)=C\left(G^{p}, M\right)=\left\{\tau: G^{p} \rightarrow M\right\}$, and the coboundary $\partial: C_{\text {group }}^{p}(G ; M) \rightarrow$ $C_{\text {group }}^{p+1}(G ; M)$ is given by

$$
\begin{aligned}
(\partial \tau)\left(g_{1}, \ldots, g_{p+1}\right)= & \tau\left(g_{2}, \ldots, g_{p+1}\right)+\sum_{i=1}^{p}(-1)^{i} \tau\left(g_{1}, \ldots, g_{i} g_{i+1}, \ldots, g_{p+1}\right) \\
& +(-1)^{p+1} \tau\left(g_{1}, \ldots, g_{p}\right) g_{p+1} .
\end{aligned}
$$

As an application of the spectral sequence, we can obtain an identification $H_{G}^{n}(\mathrm{pt} ; \mathbb{Z}) \cong$ $H_{\text {group }}^{n}(G ; \mathbb{Z})$. (We also have $H_{\text {group }}^{n}(G ; \mathbb{Z}) \cong H_{\text {group }}^{n-1}(G ; U(1))$ for $n \geq 2$ by the so-called exponential exact sequence.)

For a better geometric understanding of the spectral sequence, let us start with the fact that the Borel equivariant cohomology $H_{G}^{n}(X ; \mathbb{Z})$ is isomorphic to the cohomology $H^{n}(G \bullet \times X ; \mathbb{Z})$ of a simplicial space $G^{\bullet} \times X$ with its coefficients in the constant sheaf $\mathbb{Z}$. This is a consequence of a more general theorem about simplicial space (see [6] for example) together with the fact that the geometric realization $\left|G^{\bullet} \times X\right|$ of $G^{\bullet} \times X$ is identified with $E G \times{ }_{G} X$.

The simplicial space $G^{\bullet} \times X$ is associated to the left $G$-action on $X$, and consists of a sequence of spaces $\left\{G^{p} \times X\right\}_{p \geq 0}$ together with the face map $\partial_{i}: G^{p} \times X \rightarrow G^{p-1} \times X, i=0, \ldots, p$, and the degeneracy map $s_{i}: G^{p} \times X \rightarrow G^{p+1} \times X, i=0, \ldots, p$, given by

$$
\begin{aligned}
& \partial_{i}\left(g_{1}, \ldots, g_{p}, x\right)= \begin{cases}\left(g_{2}, \ldots, g_{p}, x\right), & i=0, \\
\left(g_{1}, \ldots, g_{i} g_{i+1}, \ldots, g_{p}, x\right), & i=1, \ldots, p-1, \\
\left(g_{1}, \ldots, g_{p-1}, g_{p} x\right), & i=p,\end{cases} \\
& s_{i}\left(g_{1}, \ldots, g_{p}, x\right)=\left(g_{1}, \ldots, g_{i-1}, 1, g_{i}, \ldots, g_{p}, x\right) .
\end{aligned}
$$

The cohomology $H^{n}\left(G^{\bullet} \times X ; \mathbb{Z}\right)$ is then defined to be the total cohomology of the double complex $\left(C^{i}\left(G^{j} \times X ; \mathbb{Z}\right), \delta, \partial\right)$, where $\left(C^{i}\left(G^{j} \times X ; \mathbb{Z}\right), \delta\right)$ is the complex computing the cohomology of $G^{j} \times X$ with coefficients in $\mathbb{Z}$ and $\partial: C^{i}\left(G^{j} \times X ; \mathbb{Z}\right) \rightarrow C^{i}\left(G^{j+1} \times X ; \mathbb{Z}\right)$ is $\partial=\sum_{i=0}^{j+1}(-1)^{i} \partial_{i}^{*}$. The double complex admits a natural filtration $\left\{\oplus_{j}{ }_{p} C^{i}\left(G^{j} \times X ; \mathbb{Z}\right)\right\}_{p \geq 0}$. The associated spectral sequence agrees with the Leray-Serre spectral sequence $E_{r}^{p, q}$, since $G$ is finite.

Now, let us consider the standard exponential exact sequence of sheaves on the simplicial space $0 \rightarrow \mathbb{Z} \rightarrow \underline{\mathbb{R}} \rightarrow U(1) \rightarrow 0$, where $\underline{\mathbb{R}}$ consists of the sheaf of $\mathbb{R}$-valued functions on $G^{p} \times X$ and $U(1)$ consists of the sheaf of $U(1)$-valued functions on $G^{p} \times X$. As in [9, Lemma 4.4], we can readily show that $H^{n}\left(G^{\bullet} \times X ; \underline{\mathbb{R}}\right)=0$ for $n>0$. This vanishing together with the associated long exact sequence leads to the following isomorphism for $n \geq 1$

$$
H^{n}\left(G^{\bullet} \times X ; \underline{U(1)}\right) \cong H^{n+1}\left(G^{\bullet} \times X ; \mathbb{Z}\right) .
$$

The cohomology $H^{n}\left(G^{\bullet} \times X ; U(1)\right)$ can be defined exactly in the same way as in the case of $H^{n}\left(G^{\bullet} \times X ; \mathbb{Z}\right)$ by using a double complex. Therefore we have a spectral sequence

$$
{ }^{\prime} E_{r}^{p, q} \Longrightarrow H^{p+q}\left(G^{\bullet} \times X ; \underline{U(1)}\right)
$$

converging to the graded quotient of a filtration

$$
{ }^{\prime} F^{0} H^{n}\left(G^{\bullet} \times X ; \underline{U(1)}\right)=H^{n}\left(G^{\bullet} \times X ; \underline{U(1)}\right) \supset^{\prime} F^{1} H^{n}\left(G^{\bullet} \times X ; \underline{U(1)}\right) \supset \cdots,
$$


whose $E_{2}$-term is

$$
{ }^{\prime} E_{2}^{p, q}=H_{\text {group }}^{p}\left(G ; H^{q}(X ; \underline{U(1)})\right),
$$

where $H^{q}(X ; U(1))$ is regarded as a right $G$-module by pull-back. It is clear that $H^{0}(X ; U(1)) \cong$ $C(X, U(1))$ and $H^{n}(X ; U(1)) \cong H^{n+1}(X ; \mathbb{Z})$ for $n \geq 1$. Since ' $E_{2}^{p, q}$ involves the group cohomology with coefficients in $C(X, U(1))$, its computation seems to be more complicated than that of $E_{2}^{p, q}$. However, the spectral sequence is useful from a geometric viewpoint, as will be seen shortly.

In view of the exponential exact sequence, the filtrations of $H_{G}^{n+1}(X ; \mathbb{Z}) \cong H^{n}(G \bullet \times X ; U(1))$ for $n \geq 1$ are related as follows

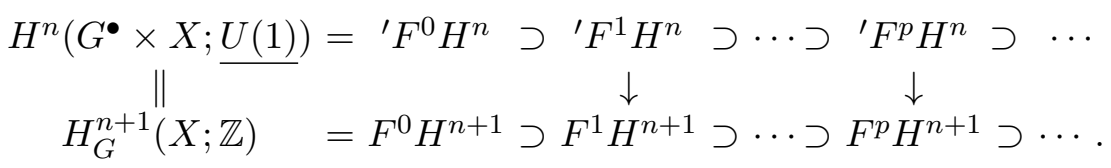

The spectral sequences are related by a map ${ }^{\prime} E_{r}^{p, q} \rightarrow E_{r}^{p, q+1}$. In particular, the $E_{2}$-terms ${ }^{\prime} E_{2}^{p, 0}$ and $E_{2}^{p, 1}$ are related by a map $C(X, U(1)) \rightarrow H^{1}(X ; \mathbb{Z})$ fitting into the exact sequence

$$
0 \rightarrow H^{0}(X ; \mathbb{Z}) \rightarrow C(X, \mathbb{R}) \rightarrow C(X, U(1)) \rightarrow H^{1}(X ; \mathbb{Z}) \rightarrow 0 .
$$

As is mentioned, because of the isomorphism $H^{q}(X ; U(1)) \cong H^{q+1}(X ; \mathbb{Z})$, we have ${ }^{\prime} E_{2}^{p, q} \cong E_{2}^{p, q+1}$ for $q \geq 1$. A more detailed relation between these spectral sequences will be given later under some hypotheses.

\subsection{Twists}

We here recall the definition of twists for equivariant $K$-theory in $[7,8]$ for the convenience of the reader. We mainly consider ungraded twists, and refer the reader to [7] for the details about graded twists (see also Remark 3.7). Recall that associated to an action of a finite group $G$ on a space $X$ is the groupoid $X / / G$ such that its set of objects is $X$ and the set of morphisms is $G \times X$.

Definition 3.1. A central extension $(L, \tau)$ of the groupoid $X / / G$ consists of the following data:

- a Hermitian line bundle $L \rightarrow G \times X$, which we write $L_{g} \rightarrow X$ for the restriction to $\{g\} \times X$ for each $g \in G$,

- unitary isomorphisms of Hermitian line bundles $\tau_{g, h}: h^{*} L_{g} \otimes L_{h} \rightarrow L_{g h}$ on $X$ for each $g, h \in G$, which we write $\tau_{g, h}(x):\left.\left.\left.L_{g}\right|_{h x} \otimes L_{h}\right|_{x} \rightarrow L_{g h}\right|_{x}$ for the restriction to $x \in X$. We assume the following diagram is commutative

$$
\begin{aligned}
& \left.\left.\left.\left.\left.L_{g}\right|_{h k x} \otimes L_{h}\right|_{k x} \otimes L_{k}\right|_{x} \stackrel{1 \otimes \tau_{h, k}(x)}{\longrightarrow} L_{g}\right|_{h k x} \otimes L_{h k}\right|_{x} \\
& \tau_{g, h}(k x) \otimes 1 \downarrow \quad \downarrow_{g, h k}(x) \\
& \left.\left.\left.L_{g h}\right|_{k x} \otimes L_{k}\right|_{x} \quad \stackrel{\tau_{g h, k}(x)}{\longrightarrow} \quad L_{g h k}\right|_{x} .
\end{aligned}
$$

Notice that if $L_{g}$ is the product line bundle, then the central extension is just a group 2-cocycle of $G$ with coefficients in $C(X, U(1))$.

Definition 3.2. An isomorphism $\left(K, \beta_{g}\right):\left(L_{g}, \tau_{g, h}\right) \rightarrow\left(L_{g}^{\prime}, \tau_{g, h}^{\prime}\right)$ of central extensions of $X / / G$ consists of the following data: 
- a Hermitian line bundle $K \rightarrow X$,

- unitary isomorphisms of Hermitian line bundles $\beta_{g}: L_{g} \otimes K \rightarrow g^{*} K \otimes L_{g}^{\prime}$ on $X$ for each $g \in G$, which we write $\beta_{g}(x):\left.\left.\left.\left.L_{g}\right|_{x} \otimes K\right|_{x} \rightarrow K\right|_{g x} \otimes L_{g}^{\prime}\right|_{x}$ for the restriction to $x \in X$. We assume the following diagram is commutative

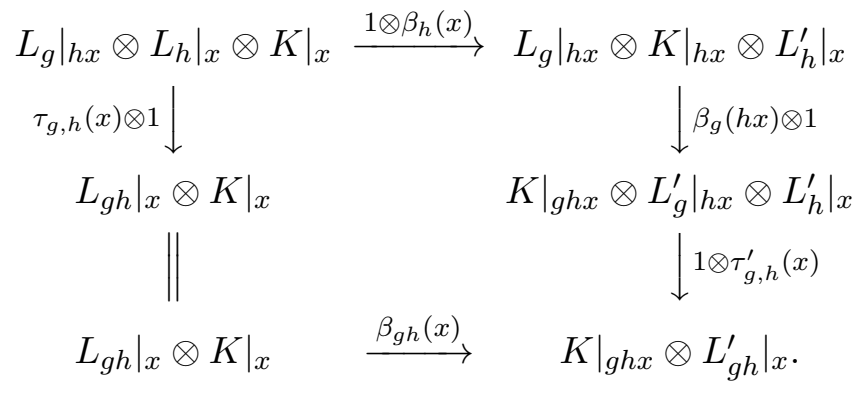

The isomorphisms $\left(K, \beta_{g}\right)$ and $\left(K^{\prime}, \beta_{g}^{\prime}\right)$ from $\left(L_{g}, \tau_{g, h}\right)$ to $\left(L_{g}^{\prime}, \tau_{g, h}^{\prime}\right)$ are identified if there is a unitary isomorphism $f: K \rightarrow K^{\prime}$ making the following diagram commutative

$$
\begin{array}{lll}
\left.\left.\left.L_{g}\right|_{x} \otimes K\right|_{x} \stackrel{\beta_{g}(x)}{\longrightarrow} K\right|_{g x} \otimes L_{g}^{\prime} \mid x & \\
1 \otimes f(x) \downarrow & & \\
\left.\left.\left.L_{g}\right|_{x} \otimes K^{\prime}\right|_{x} \stackrel{\beta_{g}^{\prime}(x)}{\longrightarrow} K^{\prime}\right|_{g x} \otimes L_{g}^{\prime} \mid x .
\end{array}
$$

Definition 3.3. An ungraded $G$-equivariant twist of $X$, or a twist for short, is a central extension of a groupoid $\tilde{X}$ which has a local equivalence to $X / / G$.

A point in this definition is that a twist needs an extra groupoid $\tilde{X}$. A central extension of $X / / G$ is a special type of a twist such that $\tilde{X}=X / / G$. Taking the extra groupoids into account, we can introduce a notion of isomorphisms to twists. We refer the reader to [7] for the details of the isomorphisms and the following classification:

Proposition 3.4 ([7]). The isomorphisms classes of ungraded $G$-equivariant twists of $X$ form an abelian group isomorphic to $H_{G}^{3}(X ; \mathbb{Z})$.

A key to the classification is the isomorphism $H_{G}^{3}(X ; \mathbb{Z}) \cong H^{2}\left(G^{\bullet} \times X ; U(1)\right)$. A close look at the proof of the classification leads to:

Lemma 3.5. The following holds true:

(i) ' ${ }^{1} H^{2}\left(G^{\bullet} \times X ; U(1)\right)$ classifies twists represented by central extensions of the groupoid $X / / G$.

(ii) ${ }^{\prime} F^{2} H^{2}(G \bullet \times X ; U(1))$ classifies twists represented by group 2-cocycles of $G$ with coefficients in the $G$-module $C(X, U(1))$.

Remark 3.6. In [8], an isomorphism of central extensions of $X / / G$ is formulated only by using the product line bundle $K=X \times \mathbb{C}$. The reason of the difference in these definitions is that we are considering an isomorphism of central extensions of $X / / G$ regarded as twists. By the same reasoning, group cocycles which are not cohomologous to each other can be isomorphic as twists.

Remark 3.7. The modification needed to define a graded twist is to replace the Hermitian line bundle $L$ constituting a central extension $(L, \tau)$ with a $\mathbb{Z}_{2}$-graded Hermitian line bundle. Since $L$ is of rank 1 , its $\mathbb{Z}_{2}$-grading amounts to specifying the degree of $L$ to be even or odd. With the suitable modification of the notion of isomorphisms, we can eventually classify graded twists by $H_{G}^{1}\left(X ; \mathbb{Z}_{2}\right) \times H_{G}^{3}(X ; \mathbb{Z})$. 


\subsection{Comparison of two spectral sequences}

The relation between the spectral sequences $E_{r}^{p, q}$ and ${ }^{\prime} E_{r}^{p, q}$ can be made more clear under a simple assumption. To present this here, we begin with a key lemma: Recall that the exponential exact sequence of sheaves on $X$ induces a natural exact sequence of right $G$-modules

$$
0 \rightarrow H^{0}(X ; \mathbb{Z}) \rightarrow C(X, \mathbb{R}) \rightarrow C(X, U(1)) \rightarrow H^{1}(X ; \mathbb{Z}) \rightarrow 0 .
$$

Let us fold this into a short exact sequence

$$
0 \rightarrow C(X, \mathbb{R}) / H^{0}(X ; \mathbb{Z}) \rightarrow C(X, U(1)) \rightarrow H^{1}(X ; \mathbb{Z}) \rightarrow 0 .
$$

In general, this does not split as an exact sequence of $G$-modules. (Such an example is provided by the circle $S^{1} \subset \mathbb{R}^{2}$ with the action of $D_{2} \subset \mathrm{O}(2)$.) Notice that if $X$ is path connected, then $H^{0}(X ; \mathbb{Z})=\mathbb{Z}$.

Lemma 3.8. If a finite group $G$ acts on a compact and path connected space $X$ fixing a point pt $\in X$, then the following exact sequence of $G$-modules splits

$$
0 \rightarrow C(X, \mathbb{R}) / \mathbb{Z} \rightarrow C(X, U(1)) \rightarrow H^{1}(X ; \mathbb{Z}) \rightarrow 0 .
$$

Proof. For notational convenience, we use the identification $U(1) \cong \mathbb{R} / \mathbb{Z}$ in this proof. Let $C(X, \mathrm{pt}, \mathbb{R}) \subset C(X, \mathbb{R})$ be the subgroup consisting of functions taking 0 at pt. The inclusion $\iota:$ pt $\rightarrow X$ induces an isomorphism of $G$-modules

$$
C(X, \mathbb{R}) \longrightarrow C(X, \mathrm{pt}, \mathbb{R}) \oplus \mathbb{R}, \quad f \mapsto\left(f-\iota^{*} f, \iota^{*} f\right) .
$$

Similarly, we have an isomorphism $C(X, \mathbb{R} / \mathbb{Z}) \cong C(X, \mathrm{pt}, \mathbb{R} / \mathbb{Z}) \oplus \mathbb{R} / \mathbb{Z}$ of $G$-modules. Thus the exact sequence of $G$-modules in question is equivalent to

$$
0 \rightarrow C(X, \mathrm{pt}, \mathbb{R}) \rightarrow C(X, \mathrm{pt}, \mathbb{R} / \mathbb{Z}) \stackrel{\delta}{\rightarrow} H^{1}(X ; \mathbb{Z}) \rightarrow 0 .
$$

Since $X$ is supposed to be compact, $H^{1}(X ; \mathbb{Z})$ is a free abelian group of finite rank. Let us choose a basis $H^{1}(X ; \mathbb{Z}) \cong \bigoplus_{i} \mathbb{Z} a_{i}$, and also $\varphi_{i}: X \rightarrow \mathbb{R} / \mathbb{Z}$ such that $\delta \varphi_{i}=a_{i}$ and $\varphi_{i}(\mathrm{pt})=0$. Modifying the splitting $a_{i} \mapsto \varphi_{i}$ of the exact sequence of abelian groups, we construct a splitting of the exact sequence of $G$-modules, which will complete the proof.

For the modification, we introduce a square matrix $A(g)=\left(A_{i j}(g)\right)$ with integer coefficients to each $g \in G$ by $g^{*} a_{i}=\sum_{j} A_{i j}(g) a_{j}$. It holds that $A(g h)=A(g) A(h)$. Because of the exact sequence, there are functions $f_{g}^{i} \in C(X, \mathrm{pt}, \mathbb{R})$ such that the following holds in $C(X, \mathrm{pt}, \mathbb{R} / \mathbb{Z})$ :

$$
g^{*} \varphi_{i}=\sum_{j} A_{i j}(g) \varphi_{j}+\left(f_{g}^{i} \bmod \mathbb{Z}\right) .
$$

This can be expressed as $g^{*} \Phi=A(g) \Phi+F_{g}$ by using the vectors $\Phi=\left(\varphi_{i}\right)$ and $F_{g}=\left(F_{g}^{i}\right)$. It then holds that $F_{g h}=A(g) F_{h}+h^{*} F_{g}$ in $C(X, \mathrm{pt}, \mathbb{R})$. Since $A(g)$ is invertible, this is equivalent to

$$
A(g h)^{-1} F_{g h}=A(h)^{-1} F_{h}+A(h)^{-1} h^{*}\left(A(g)^{-1} F_{g}\right) .
$$

Write $|G|$ for the order of $G$, and put $\bar{F}=\frac{1}{|G|} \sum_{g \in G} A(g)^{-1} F_{g}$. Taking the average over $g \in G$ in the formula above, we get

$$
\bar{F}=A(h)^{-1} F_{h}+A(h)^{-1} h^{*} \bar{F},
$$

which is equivalent to $F_{g}=A(g) \bar{F}-g^{*} \bar{F}$. Now $g^{*}(\Phi+\bar{F})=A(g)(\Phi+\bar{F})$. Thus, under the expression $\bar{F}=\left(\bar{f}^{i}\right)$ by using $\bar{f}^{i} \in C(X, \mathrm{pt}, \mathbb{R})$, the assignment $a_{i} \mapsto \varphi_{i}+\left(\bar{f}^{i} \bmod \mathbb{Z}\right)$ defines a splitting $H^{1}(X ; \mathbb{Z}) \rightarrow C(X, \mathrm{pt}, \mathbb{R} / \mathbb{Z})$ compatible with the $G$-module structures. 
Lemma 3.9. Let $G$ be a finite group acting on a compact and path connected space $X$ fixing a point $\mathrm{pt} \in X$. Then, for $n \geq 1$, there is an isomorphism

$$
H_{\text {group }}^{n}(G ; C(X, U(1))) \cong H_{\text {group }}^{n}(G ; U(1)) \oplus H_{\text {group }}^{n}\left(G ; H^{1}(X ; \mathbb{Z})\right),
$$

where $U(1)$ is the trivial $G$-module, and $H^{1}(X ; \mathbb{Z})$ is regarded as a $G$-module through the action of $G$ on $X$.

Proof. Lemma 3.8 implies

$$
H_{\text {group }}^{n}(G ; C(X, U(1))) \cong H_{\text {group }}^{n}(G ; C(X, \mathbb{R}) / \mathbb{Z}) \oplus H_{\text {group }}^{n}\left(G ; H^{1}(X ; \mathbb{Z})\right)
$$

for all $n \geq 1$. By the $G$-module isomorphism $C(X, \mathbb{R}) / \mathbb{Z} \cong C(X, \mathrm{pt}, \mathbb{R}) \oplus \mathbb{R} / \mathbb{Z}$ utilized in Lemma 3.8, we have

$$
H_{\text {group }}^{n}(G ; C(X, \mathbb{R}) / \mathbb{Z}) \cong H_{\text {group }}^{n}(G ; C(X, \mathrm{pt}, \mathbb{R})) \oplus H_{\text {group }}^{n}(G ; \mathbb{R} / \mathbb{Z})
$$

Since $C(X, \mathrm{pt}, \mathbb{R})$ is a vector space over $\mathbb{R}$, we can prove the vanishing $H_{\text {group }}^{n}(G ; C(X, \mathrm{pt}, \mathbb{R}))=0$ for $n \geq 1$ by an average argument as in [9, Lemma 4.4].

Proposition 3.10. Suppose that a finite group $G$ acts on a compact and path connected space $X$ fixing a point $\mathrm{pt} \in X$. Then for $r \geq 2$ we have

$$
{ }^{\prime} E_{r}^{p, 0} \cong E_{r}^{p, 1} \oplus E_{r}^{p+1,0}, \quad p \geq 1, \quad{ }^{\prime} E_{r}^{p, q} \cong E_{r}^{p, q+1}, \quad p \geq 0, q \geq 1 .
$$

Proof. Recall that the exponential exact sequence induces the connecting homomorphism $\delta: H^{q}(X ; U(1)) \rightarrow H^{q+1}(X ; \mathbb{Z})$ and this induces a natural homomorphism $\delta:{ }^{\prime} E_{r}^{p, q} \rightarrow E_{r}^{p, q+1}$ compatible with the differentials ' $d_{r}$ and $d_{r}$. In the case of $r=2$, the homomorphism $\delta:{ }^{\prime} E_{2}^{p, q} \rightarrow$ $E_{2}^{p, q+1}$ is bijective for $q \geq 1$ and $p \geq 0$, and we have ' $E_{2}^{p, 0} \cong E_{2}^{p, 1} \oplus E_{2}^{p+1,0}$ for $p \geq 1$ as a consequence of Lemma 3.9. Notice that, under this isomorphism, $\delta:{ }^{\prime} E_{2}^{p, 0} \rightarrow E_{2}^{p, 1}$ for $p \geq 1$ restricts to the identity on the direct summand $E_{2}^{p, 1} \subset{ }^{\prime} E_{2}^{p, 0}$. Note also that $E_{2}^{p, 0}=E_{\infty}^{p, 0}$ for any $p$, because

$$
E_{2}^{p, 0}=H_{\text {group }}^{p}(G ; \mathbb{Z})=H^{p}(B G ; \mathbb{Z})=H_{G}^{p}(\mathrm{pt} ; \mathbb{Z})
$$

is a direct summand of $H_{G}^{p}(X ; \mathbb{Z}) \cong H_{G}^{0}(\mathrm{pt} ; \mathbb{Z}) \oplus \tilde{H}_{G}^{p}(X ; \mathbb{Z})$, where $\tilde{H}_{G}^{p}(X ; \mathbb{Z})$ is the reduced cohomology. Thus, for $p \geq 1$, the map $\delta:{ }^{\prime} E_{2}^{p, 0} \rightarrow E_{2}^{p, 1}$ is the projection onto $E_{2}^{p, 1}$ and the image of the differential ' $d_{2}:{ }^{\prime} E_{2}^{p-2,1} \rightarrow{ }^{\prime} E_{2}^{p, 0}$ is in the direct summand $E_{2}^{p, 0}$. This leads to

$$
{ }^{\prime} E_{3}^{p, 0} \cong E_{3}^{p, 0} \oplus E_{3}^{p, 1}, \quad p \geq 1, \quad{ }^{\prime} E_{3}^{p, q} \cong E_{3}^{p, q+1}, \quad p \geq 0, q \geq 1 .
$$

The calculation above can be repeated inductively on $r$.

Corollary 3.11. Let $G$ and $X$ be as in Proposition 3.10. Then, for any $n \geq 1$ and $p=0, \ldots, n$, there is a natural isomorphism

$$
{ }^{\prime} F^{p} H^{n}\left(G^{\bullet} \times X ; \underline{U(1)}\right) \cong F^{p} H_{G}^{n+1}(X ; \mathbb{Z}) .
$$

In addition, we have a decomposition

$$
{ }^{\prime} F^{n} H^{n}(G \bullet \times X: \underline{U(1)}) \cong F^{n} H_{G}^{n+1}(X ; \mathbb{Z}) \cong E_{\infty}^{n, 1} \oplus F^{n+1} H_{G}^{n+1}(X ; \mathbb{Z}),
$$

in which $F^{n+1} H_{G}^{n+1}(X ; \mathbb{Z}) \cong H_{G}^{n+1}(\mathrm{pt} ; \mathbb{Z}) \cong H_{\text {group }}^{n}(G ; U(1))$. 
Proof. Put ${ }^{\prime} F^{p} H^{n}={ }^{\prime} F^{p} H^{n}\left(G^{\bullet} \times X ; U(1)\right)$ and $F^{p} H^{n+1}=F^{p} H_{G}^{n+1}(X ; \mathbb{Z})$ for short. The exponential exact sequence induces a homomorphism of short exact sequences

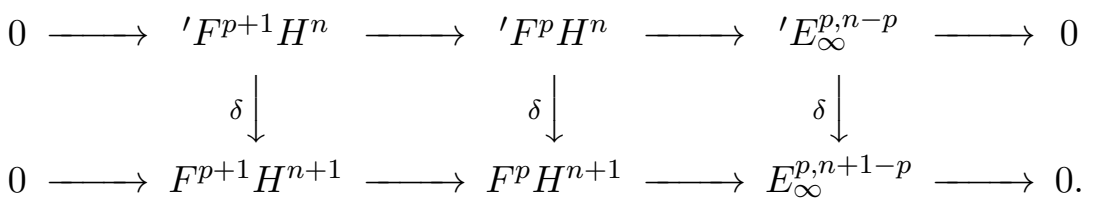

In the case of $p=n$, the diagram above becomes

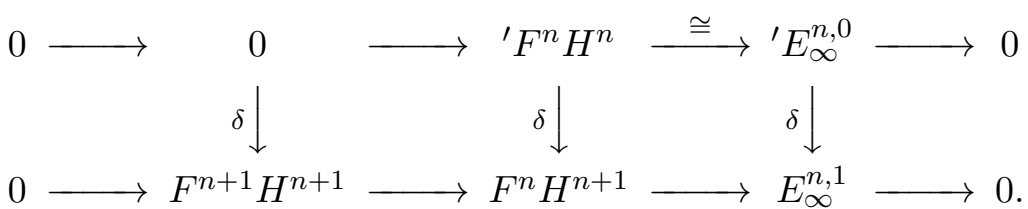

Notice that $F^{n+1} H^{n+1} \cong E_{\infty}^{n+1,0} \cong E_{2}^{n+1,0}$ since $E_{2}^{n+1,0} \cong H_{G}^{n+1}(\mathrm{pt} ; \mathbb{Z})$ must survive into $H_{G}^{n+1}(X ; \mathbb{Z}) \cong H_{G}^{n+1}(\mathrm{pt} ; \mathbb{Z}) \oplus \tilde{H}_{G}^{n+1}(X ; \mathbb{Z})$. Hence $F^{n} H^{n+1} \cong E_{\infty}^{n+1,0} \oplus E_{\infty}^{n, 1}$. If $n \geq 1$, then this isomorphism is compatible with the isomorphism ' $E_{\infty}^{n, 0} \cong E_{\infty}^{n+1,0} \oplus E_{\infty}^{n, 1}$ in Proposition 3.10 through $\delta$, so that

$$
{ }^{\prime} F^{n} H^{n} \stackrel{\delta}{\cong} F^{n} H^{n+1} \cong F^{n+1} H^{n+1} \oplus E_{\infty}^{n, 1} .
$$

For $p=n-1, n-2, \ldots, 1,0$, we know $\delta:{ }^{\prime} E_{\infty}^{p, n-p} \rightarrow E_{\infty}^{p, n-p+1}$ is bijective by Proposition 3.10. Therefore ' $F^{p} H^{n} \cong F^{p} H^{n+1}$ inductively.

Combining the above corollary with Lemma 3.5, we get the interpretations of $F^{p} H_{G}^{3}(X ; \mathbb{Z})$ by twists presented in Introduction:

Corollary 3.12. Let $G$ and $X$ be as in Proposition 3.10 .

(i) $F^{1} H_{G}^{3}(X ; \mathbb{Z})$ classifies twists which can be represented by central extensions of the groupoid $X / / G$.

(ii) $F^{2} H_{G}^{3}(X ; \mathbb{Z})$ classifies twists which can be represented by 2-cocycles of $G$ with coefficients in the $G$-module $C(X, U(1))$.

(iii) $F^{3} H_{G}^{3}(X ; \mathbb{Z})=H_{\text {group }}^{2}(G ; U(1))$ classifies twists which can be represented by 2 -cocycles of $G$ with coefficients in the trivial $G$-module $U(1)$.

Remark 3.13. The coincidence ' $F^{1} H^{n}\left(G^{\bullet} \times X ; U(1)\right)=F^{1} H^{n+1}(X ; \mathbb{Z})$ in Corollary 3.11 holds

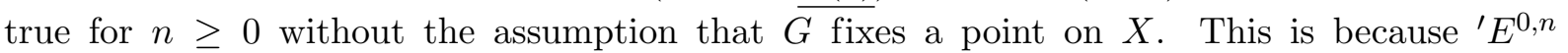
and $E^{0, n+1}$ are subgroups of $H^{n}(X ; U(1)) \cong H^{n+1}(X ; \mathbb{Z})$ and it holds that

$$
\begin{aligned}
{ }^{\prime} F^{1} H^{n}\left(G^{\bullet} \times X ; \underline{U(1)}\right) & =F^{1} H^{n+1}(X ; \mathbb{Z}) \\
& =\operatorname{Ker}\left[f: H^{n}(G \bullet \times X ; \underline{U(1)}) \cong H_{G}^{n+1}(X ; \mathbb{Z}) \rightarrow H^{n+1}(X ; \mathbb{Z})\right],
\end{aligned}
$$

where $f$ is the homomorphism of "forgetting the group actions".

\section{The proof of Theorems 1.1 and 1.3}

Theorems 1.1 and 1.3 are proved here based on case-by-case computations of the equivariant cohomology and the Leray-Serre spectral sequence. Some basic facts that are useful for the computation are summarized first. We then provide the outline of the computations and details of typical cases, p2, p4m/p4g and p6m. Finally, Corollary 1.2 is proved. 


\subsection{Some generality}

The cohomology $H^{n}\left(T^{2} ; \mathbb{Z}\right)$ of the torus is well known, so that nothing remains to be proven in the case of $\mathrm{p} 1$.

For the point group $P$ of any 2 -dimensional space group, the vanishing $H^{3}\left(T^{2} ; \mathbb{Z}\right)=0$ implies $E_{\infty}^{0,3}=0$, so that

$$
H_{P}^{3}\left(T^{2} ; \mathbb{Z}\right)=F^{0} H_{P}^{3}\left(T^{2} ; \mathbb{Z}\right)=F^{1} H_{P}^{3}\left(T^{2} ; \mathbb{Z}\right)
$$

Note that each point group $P$ fixes a point on $T^{2}$, so that

$$
F^{3} H_{P}^{3}\left(T^{2} ; \mathbb{Z}\right)=H_{P}^{3}(\mathrm{pt} ; \mathbb{Z})=H_{\text {group }}^{3}(P ; \mathbb{Z})=H_{\text {group }}^{2}(P ; U(1)) .
$$

Then the main task for the proof of Theorem 1.1 is to compute $H_{P}^{3}\left(T^{2} ; \mathbb{Z}\right)$ and $F^{2} H_{P}^{3}\left(T^{2} ; \mathbb{Z}\right)$, since in the case where $P$ is the cyclic group $\mathbb{Z}_{n}$ or the dihedral group $D_{n}$, the cohomology $H_{P}^{m}(\mathrm{pt} ; \mathbb{Z})$ is summarized as follows:

\begin{tabular}{|c|c|c|c|c|}
\hline$P$ & $H_{P}^{0}(\mathrm{pt} ; \mathbb{Z})$ & $H_{P}^{1}(\mathrm{pt} ; \mathbb{Z})$ & $H_{P}^{2}(\mathrm{pt} ; \mathbb{Z})$ & $H_{P}^{3}(\mathrm{pt} ; \mathbb{Z})$ \\
\hline $\mathbb{Z}_{n}$ & $\mathbb{Z}$ & 0 & $\mathbb{Z}_{n}$ & 0 \\
\hline$D_{n}$ & $\mathbb{Z}$ & 0 & $\begin{cases}\mathbb{Z}_{2} & (n: \text { odd }) \\
\mathbb{Z}_{2}^{\oplus 2} & (n: \text { even })\end{cases}$ & $\begin{cases}0 & (n: \text { odd }) \\
\mathbb{Z}_{2} & (n: \text { even })\end{cases}$ \\
\hline
\end{tabular}

The degree 0 part $H_{P}^{0}(\mathrm{pt} ; \mathbb{Z})=H^{0}(B P ; \mathbb{Z})=\mathbb{Z}$ is clear. Since $P$ is finite, the degree 1 part $H_{P}^{1}(\mathrm{pt} ; \mathbb{Z}) \cong \operatorname{Hom}(P, \mathbb{Z})$ is trivial. The degree 2 part $H_{P}^{2}(\mathrm{pt} ; \mathbb{Z}) \cong \operatorname{Hom}(P, U(1))$ can be seen by the classification of irreducible representations. Finally, the degree 3 part $H_{P}^{3}(\mathrm{pt} ; \mathbb{Z}) \cong$ $H_{\text {group }}^{2}(P ; U(1))$ for $P=\mathbb{Z}_{n}, D_{n}$ can be found in [14].

In the rest of the section, we may use a structure of $T^{2}$ as a $P$-CW complex. In general, for a compact Lie group $G$, a $G-C W$ complex is an analogue of a CW complex made of $G$-cells. A $d$-dimensional $G$-cell is a $G$-space of the form $G / H \times e^{d}$, where $H \subset G$ is a closed subgroup and $e^{d}$ is the standard $d$-dimensional cell. The $G$-action on $G / H$ is the left translation, whereas that on $e^{d}$ is trivial. For the details, we refer the reader to [19].

We later compute a group cohomology via cohomology of a space:

Lemma 4.1. Suppose that a finite group $G$ acts on a path connected space $Y$ fixing at least one point $\mathrm{pt} \in Y$. Suppose further that $Y$ is a $C W$ complex consisting of only cells of dimension less than or equal to 1 . Then the following holds true for all $n \geq 0$.

$$
H_{\text {group }}^{n}\left(G ; H^{1}(Y ; \mathbb{Z})\right) \cong \tilde{H}_{G}^{n+1}(Y ; \mathbb{Z})
$$

where $\tilde{H}_{G}^{n+1}(Y ; \mathbb{Z})$ stands for the reduced cohomology.

Notice that a $G$-CW complex is naturally a $\mathrm{CW}$ complex.

Proof. Consider the Leray-Serre spectral sequence

$$
E_{2}^{p, q}=H_{\text {group }}^{p}\left(G ; H^{q}(Y ; \mathbb{Z})\right) \Longrightarrow H_{G}^{*}(Y ; \mathbb{Z}) .
$$

Note that $H^{q}(Y ; \mathbb{Z})=0$ for $q \neq 0,1$. The $E_{2}$-term $E_{2}^{p, 0}=H_{\text {group }}^{p}(G ; \mathbb{Z})$ must survive into the direct summand $H_{G}^{p}(\mathrm{pt} ; \mathbb{Z})$ in $H_{G}^{p}(Y ; \mathbb{Z}) \cong H_{G}^{p}(\mathrm{pt} ; \mathbb{Z}) \oplus \tilde{H}_{G}^{p}(Y ; \mathbb{Z})$. Therefore it must hold that

$$
\tilde{H}_{G}^{p}(Y ; \mathbb{Z}) \cong E_{\infty}^{p-1,1}=E_{2}^{p-1,1} \cong H_{\text {group }}^{p-1}\left(G ; H^{1}(Y ; \mathbb{Z})\right)
$$

which completes the proof. 
We also prepare a simple lemma about group cohomology: Let $G$ be a finite group, $c: G \rightarrow$ $\mathbb{Z}_{2}=\{ \pm 1\}$ a surjective homomorphism, and $\tilde{\mathbb{Z}}=\mathbb{Z}_{c}$ the $G$-module such that its underlying group is $\mathbb{Z}$ and $G$ acts (from the right) by $m \mapsto m c(g)$. A typical example is a finite subgroup $P \subset \mathrm{O}(2)$ such that $P \not \subset \mathrm{SO}(2)$ with $c$ the composition of the inclusion $P \rightarrow \mathrm{O}(2)$ and the determinant $\mathrm{O}(2) \rightarrow \mathbb{Z}_{2}$.

Lemma 4.2. Let $G$, $c$ and $\tilde{\mathbb{Z}}$ be as above. Then, $H_{\text {group }}^{0}(G ; \tilde{\mathbb{Z}})=0$ and $H_{\text {group }}^{1}(G ; \tilde{\mathbb{Z}}) \cong \mathbb{Z}_{2}$.

Proof. For any $n \in C_{\text {group }}^{0}(G ; \tilde{\mathbb{Z}})=\mathbb{Z}$, its coboundary $\partial n: G \rightarrow \mathbb{Z}$ is $(\partial n)(g)=n(1-c(g))$. Thus, the assumption that $c$ is surjective implies the vanishing of the 0th cohomology. The inclusion $\operatorname{Ker}(c) \subset G$ induces an injection on 1-cocycles

$$
Z_{\text {group }}^{1}(G ; \tilde{\mathbb{Z}}) \rightarrow Z_{\text {group }}^{1}(\operatorname{Ker}(c) ; \tilde{\mathbb{Z}})=\operatorname{Hom}(\operatorname{Ker}(c), \mathbb{Z})=0 .
$$

Thus, given a group 1-cocycle $\phi \in Z_{\text {group }}^{1}(G ; \tilde{\mathbb{Z}})$, it holds that $\phi(g)=0$ for all $g \in \operatorname{Ker}(c)$. If $g, h \notin \operatorname{Ker}(c)$, then the cocycle condition $(\partial \phi)(g, h)=0$ implies $\phi(g)=\phi(h)$. Therefore $\phi: G \rightarrow \mathbb{Z}$ is always of the form $\phi(g)=n(1-c(g)) / 2$ for some $n \in \mathbb{Z}$. This provides the identification $Z_{\text {group }}^{1}(G ; \tilde{\mathbb{Z}}) \cong \mathbb{Z}$ as well as $B_{\text {group }}^{1}(G ; \tilde{\mathbb{Z}}) \cong 2 \mathbb{Z}$, which completes the proof.

In some cases, the computations of the Leray-Serre spectral sequence are similar, which we summarize as follows:

Lemma 4.3. Let $G$ be a finite group acting on the torus $T^{2}$ such that:

- there is a fixed point $\mathrm{pt} \in T^{2}$,

- the $G$-action does not preserve the orientation of $T^{2}$.

Then the following holds true about the Leray-Serre spectral sequence:

(a) $F^{2} H_{G}^{3}\left(T^{2} ; \mathbb{Z}\right) \cong E_{2}^{2,1} \oplus E_{2}^{3,0}$,

(b) $H_{G}^{n}\left(T^{2} ; \mathbb{Z}\right) \cong \bigoplus_{p+q=n} E_{2}^{p, q}$ for $n \leq 2$.

Proof. In the Leray-Serre spectral sequence $E_{2}^{p, q}=H_{\text {group }}^{p}\left(G ; H^{q}\left(T^{2} ; \mathbb{Z}\right)\right)$, the coefficient in the group cohomology $H^{0}\left(T^{2}\right) \cong \mathbb{Z}$ is identified with the trivial $G$-module, and $H^{2}\left(T^{2}\right) \cong \mathbb{Z}$ with the $G$-module in Lemma 4.2 . Then the relevant $E_{2}$-terms can be summarized as follows:

\begin{tabular}{|c|c|c|c|c|c|}
\hline$q=3$ & 0 & 0 & 0 & 0 & 0 \\
\hline$q=2$ & 0 & $\mathbb{Z}_{2}$ & & & \\
\hline$q=1$ & $E_{2}^{0,1}$ & $E_{2}^{1,1}$ & $E_{2}^{2,1}$ & & \\
\hline$q=0$ & $E_{2}^{0,0}$ & $E_{2}^{1,0}$ & $E_{2}^{2,0}$ & $E_{2}^{3,0}$ & $E_{2}^{4,0}$ \\
\hline$E_{2}^{p, q}$ & $p=0$ & $p=1$ & $p=2$ & $p=3$ & $p=4$ \\
\hline
\end{tabular}

Since $G$ fixes pt $\in T^{2}$, we have the decomposition $H_{G}^{n}\left(T^{2} ; \mathbb{Z}\right) \cong H_{G}^{n}(\mathrm{pt} ; \mathbb{Z}) \oplus \tilde{H}_{G}^{n}\left(T^{2} ; \mathbb{Z}\right)$, where $\tilde{H}_{G}^{n}\left(T^{2} ; \mathbb{Z}\right)$ is the reduced cohomology. Therefore the $E_{2}$-term $E_{2}^{n, 0}=H_{\text {group }}^{n}(G ; \mathbb{Z}) \cong H_{G}^{n}(\mathrm{pt} ; \mathbb{Z})$ must survive into the direct summand $H_{G}^{n}(\mathrm{pt} ; \mathbb{Z})$ in $H_{G}^{n}\left(T^{2} ; \mathbb{Z}\right)$. This implies that $E_{2}^{n, 0}=E_{\infty}^{n, 0}$ is always a direct summand of the subgroups $F^{p} H_{G}^{n}\left(T^{2} ; \mathbb{Z}\right) \subset H_{G}^{n}\left(T^{2} ; \mathbb{Z}\right)$ and that $d_{2}: E_{2}^{p-2,1} \rightarrow$ $E_{2}^{p, 0}$ is trivial. As a result, we get $E_{2}^{2,1}=E_{\infty}^{2,1}$ and the isomorphism (a). Also $E_{2}^{p, q}=E_{\infty}^{p, q}$ for $p+q \leq 2$, and the isomorphism (b) follows.

The degeneration of the spectral sequence in the above lemma can be generalized in some cases. For this aim, the key is the following equivariant stable splitting of $T^{2}$ (cf. [8, Theorem 11.8]). 
Lemma 4.4. Suppose a finite group $G$ acts on the torus $T^{2}=S^{1} \times S^{1}$ and

- there is a fixed point $\mathrm{pt}=\left(x_{0}, y_{0}\right) \in T^{2}$ under the $G$-action,

- $G$ preserves the subspace $S^{1} \vee S^{1}=S^{1} \times\left\{y_{0}\right\} \cup\left\{x_{0}\right\} \times S^{1} \subset T^{2}$.

Then there is a G-equivariant homotopy equivalence

$$
\Sigma T^{2} \simeq \Sigma\left(S^{1} \vee S^{1}\right) \vee \Sigma\left(T^{2} / S^{1} \vee S^{1}\right)
$$

where $\Sigma$ stands for the reduced suspension.

Proof. The argument of the proof of Proposition 4I.1 [11, p. 467] can be applied to our equivariant case.

We remark that the point groups of the 2-dimensional space groups without elements of order 3 fulfill the assumptions of the lemma above.

Lemma 4.5. Under the assumption in Lemma 4.4, we have the following isomorphism of groups for all $n \in \mathbb{Z}$

$$
H_{G}^{n}\left(T^{2} ; \mathbb{Z}\right) \cong H_{G}^{n}(\mathrm{pt} ; \mathbb{Z}) \oplus \tilde{H}_{G}^{n}\left(S^{1} \vee S^{1} ; \mathbb{Z}\right) \oplus \tilde{H}_{G}^{n}\left(T^{2} / S^{1} \vee S^{1} ; \mathbb{Z}\right)
$$

Further, the Leray-Serre spectral sequence for $H_{G}^{n}\left(T^{2} ; \mathbb{Z}\right)$ degenerates at $E_{2}$ and the relevant extension problems are trivial, so that

(a) $F^{2} H_{G}^{3}\left(T^{2} ; \mathbb{Z}\right) \cong E_{2}^{2,1} \oplus E_{2}^{3,0}$,

(b) $H_{G}^{n}\left(T^{2} ; \mathbb{Z}\right) \cong \bigoplus_{p+q=n} E_{2}^{p, q}$ for all $n \in \mathbb{Z}$.

Proof. The stable splitting in Lemma 4.4 immediately gives the first isomorphism. For the trivial $G$-space pt, the Leray-Serre spectral sequence clearly degenerates at $E_{2}$, and we have

$$
H_{G}^{n}(\mathrm{pt} ; \mathbb{Z}) \cong H_{\text {group }}^{n}\left(G ; H^{0}(\mathrm{pt} ; \mathbb{Z})\right) \text {. }
$$

Since $H^{0}(\mathrm{pt} ; \mathbb{Z}) \cong H^{0}\left(T^{2} ; \mathbb{Z}\right)$ as $G$-modules, we get the following identification of the $E_{2^{-}}$ term $E_{2}^{n, 0}$ of the Leray-Serre spectral sequence for $H_{G}^{n}\left(T^{2} ; \mathbb{Z}\right)$

$$
E_{2}^{n, 0}=H_{\text {group }}^{n}\left(G ; H^{0}\left(T^{2} ; \mathbb{Z}\right)\right) \cong H_{G}^{n}(\mathrm{pt} ; \mathbb{Z}) .
$$

For the $G$-space $S^{1} \vee S^{1}$, we can see, as in the proof of Lemma 4.1, that the Leray-Serre spectral sequence also degenerates at $E_{2}$ and the extension problems are trivial. Because $H^{1}\left(S^{1} \vee S^{1} ; \mathbb{Z}\right) \cong$ $H^{1}\left(T^{2} ; \mathbb{Z}\right)$ as $G$-modules, the $E_{2}$-term $E_{2}^{n-1,1}$ of the Leray-Serre spectral sequence for $H_{G}^{n}\left(T^{2} ; \mathbb{Z}\right)$ is

$$
E_{2}^{n-1,1}=H_{\text {group }}^{n-1}\left(G ; H^{1}\left(T^{2} ; \mathbb{Z}\right)\right) \cong \tilde{H}_{G}^{n}\left(S^{1} \vee S^{1} ; \mathbb{Z}\right)
$$

Exactly in the same way, we have

$$
E_{2}^{n-2,2}=H_{\text {group }}^{n-2}\left(G ; H^{2}\left(T^{2} ; \mathbb{Z}\right)\right) \cong \tilde{H}_{G}^{n}\left(T^{2} / S^{1} \vee S^{1} ; \mathbb{Z}\right),
$$

since $H^{2}\left(T^{2} / S^{1} \vee S^{1} ; \mathbb{Z}\right) \cong H^{2}\left(T^{2} ; \mathbb{Z}\right)$ as $G$-modules. The first isomorphism now gives $H_{G}^{n}\left(T^{2} ; \mathbb{Z}\right)$ $\cong E_{2}^{n, 0} \oplus E_{2}^{n-1,1} \oplus E_{2}^{n-2,2}$, which also implies the triviality of the spectral sequence. 


\subsection{The outline of computations}

Theorems 1.1 and 1.3 follow from case by case computations. As mentioned in Section 1, three methods are applicable.

1. In the cases of $\mathrm{p} 2$ and $\mathrm{pm} / \mathrm{pg}$, the point group $\mathbb{Z}_{2}=D_{1}$ acts on the torus $T^{2}=S^{1} \times S^{1}$ preserving the direct product structure, so that we can think of $T^{2}$ as a stack of certain equivariant circle bundles $\left(\mathbb{Z}_{2}\right.$-equivariant principal circle bundles and/or 'Real' circle bundles in the sense of [10]). For such circle bundles, we can use the Gysin exact sequence to compute the equivariant cohomology, as detailed in [10]. In particular, in the cases of $\mathrm{p} 2$ and $\mathrm{pm} / \mathrm{pg}$, the Gysin exact sequences are split, and the computations are very simple. (The computation by using the Gysin sequence is also valid for $\mathrm{cm}$, even though the sequence is non-split.) In the case of p2, we do not need to compute the Leray-Serre spectral sequence, since the third cohomology is trivial. In the case of $\mathrm{pm} / \mathrm{pg}$, the spectral sequence can be computed directly.

2. In the cases of $\mathrm{p} 4, \mathrm{~cm}, \mathrm{pmm} / \mathrm{pmg} / \mathrm{pgg}, \mathrm{cmm}$ and $\mathrm{p} 4 \mathrm{~m} / \mathrm{p} 4 \mathrm{~g}$, we can verify that the action of the point group on $T^{2}$ satisfies the assumptions of Lemma 4.4, by inspecting the explicit presentation in Appendix A. Hence we can apply Lemma 4.5 to the computation of the equivariant cohomology and the spectral sequence. In this application, the only non-trivial part is the equivariant cohomology of the invariant subspace $S^{1} \vee S^{1}$, which we compute by using the Mayer-Vietoris exact sequence.

3. In the cases of $\mathrm{p} 3, \mathrm{p} 6, \mathrm{p} 3 \mathrm{~m} 1, \mathrm{p} 31 \mathrm{~m}$ and $\mathrm{p} 6 \mathrm{~m}$, the computation can be divided into two parts. One part is to compute $H_{P}^{3}\left(T^{2} ; \mathbb{Z}\right)$. This is carried out by taking a $P$-CW decomposition of $T^{2}$, and by using the Mayer-Vietoris exact sequence and the exact sequence for a pair. The other part is to compute the Leray-Serre spectral sequence. In this part, we need to know the group cohomology with coefficients in $H^{1}\left(T^{2} ; \mathbb{Z}\right)$. For this aim, we take an invariant subspace $Y \subset T^{2}$ of one dimension. The equivariant cohomology of $Y$ is computed by using Mayer-Vietoris sequence, which allows us to know the group cohomology with its coefficients in $H^{1}(Y ; \mathbb{Z})$ through Lemma 4.1. The coefficients $H^{1}(Y ; \mathbb{Z})$ and $H^{1}\left(T^{2} ; \mathbb{Z}\right)$ are related by a short exact sequence. The associated long exact sequence then computes the group cohomology with coefficients in $H^{1}\left(T^{2} ; \mathbb{Z}\right)$. Depending on the cases, one of these parts happens to be enough to complete the computation.

In the cases of $\mathrm{p} 2, \mathrm{p} 4 \mathrm{~m} / \mathrm{p} 4 \mathrm{~g}$ and $\mathrm{p} 6 \mathrm{~m}$, the detail of the computation is provided in the following subsections. The details for the other cases can be found in old versions of arXiv:1509.09194.

\section{$4.3 \quad \mathrm{p} 2$}

The lattice $\Pi \subset \mathbb{R}^{2}$ is the standard one $\Pi=\mathbb{Z} \oplus \mathbb{Z}$ and the point group $P=\mathbb{Z}_{2}=\{ \pm 1\}$ acts on $\Pi$ and $\mathbb{R}^{2}$ by $(x, y) \mapsto(-x,-y)$.

Theorem 4.6 (p2). The $\mathbb{Z}_{2}$-equivariant cohomology of $T^{2}$ is given as follows

\begin{tabular}{|c|c|c|c|c|}
\hline & $n=0$ & $n=1$ & $n=2$ & $n=3$ \\
\hline$H_{\mathbb{Z}_{2}}^{n}\left(T^{2} ; \mathbb{Z}\right)$ & $\mathbb{Z}$ & 0 & $\mathbb{Z} \oplus \mathbb{Z}_{2}^{\oplus 3}$ & 0 \\
\hline
\end{tabular}

Proof. We use the Gysin exact sequence for 'Real' circle bundles in [10]: We write $H_{\mathbb{Z}_{2}}^{n}(X)=$ $H_{\mathbb{Z}_{2}}^{n}(X ; \mathbb{Z})$ for the equivariant cohomology and $H_{ \pm}^{n}(X) \cong H_{\mathbb{Z}}^{n}(X ; \mathbb{Z}(1))$ for a variant of the equivariant cohomology, which can be formulated by the equivariant cohomology with local coefficients. The torus $T^{2}$ is the product of two copies of $\tilde{S}^{1}$, where $\tilde{S}^{1}=U(1)$ is the circle with the involution $z \mapsto z^{-1}$. We can think of $\tilde{S}^{1} \times \tilde{S}^{1}$ as the trivial 'Real' circle bundle on $\tilde{S}^{1}$. 
K. Gomi

Similarly, $\tilde{S}^{1}$ is the trivial 'Real' circle bundle on pt. The Gysin exact sequences for these 'Real' circle bundles are split, and we find

$$
H_{\mathbb{Z}_{2}}^{n}\left(T^{2}\right) \cong H_{\mathbb{Z}_{2}}^{n}\left(\tilde{S}^{1}\right) \oplus H_{ \pm}^{n-1}\left(\tilde{S}^{1}\right) \cong H_{\mathbb{Z}_{2}}^{n}(\mathrm{pt}) \oplus H_{ \pm}^{n-1}(\mathrm{pt}) \oplus H_{ \pm}^{n-1}(\mathrm{pt}) \oplus H_{\mathbb{Z}_{2}}^{n-2}(\mathrm{pt})
$$

As given in [10], the cohomology $H_{ \pm}^{n}(\mathrm{pt})$ is isomorphic to $\mathbb{Z}_{2}$ if $n>0$ is odd, and is trivial otherwise. We already know $H_{\mathbb{Z}_{2}}^{n}(\mathrm{pt})$, and get $H_{\mathbb{Z}_{2}}^{n}\left(T^{2}\right)$ easily.

\section{$4.4 \mathrm{p} 4 \mathrm{~m} / \mathrm{p} 4 \mathrm{~g}$}

The lattice $\Pi=\mathbb{Z}^{2} \subset \mathbb{R}^{2}$ is standard. The point group is $P=D_{4}=\left\langle C_{4}, \sigma_{x} \mid C_{4}^{4}, \sigma_{x}^{2}, \sigma_{x} C_{4} \sigma_{x} C_{4}\right\rangle$. The $D_{4}$-action on $\Pi$ and $\mathbb{R}^{2}$ is given by the following matrix presentation:

$$
C_{4}=\left(\begin{array}{cc}
0 & -1 \\
1 & 0
\end{array}\right), \quad \sigma_{x}=\left(\begin{array}{cc}
-1 & 0 \\
0 & 1
\end{array}\right)
$$

In the rest of this subsection, we will use the following notations to indicate elements in $D_{4}$ :

$$
\begin{array}{llll}
1, & C_{4}, & C_{2}=C_{4}^{2}, & C_{4}^{-1}=C_{4}^{3}, \\
\sigma_{x}, & \sigma_{d}=\sigma_{x} C_{4}, & \sigma_{y}=C_{2} \sigma_{x}, & \sigma_{d}^{\prime}=C_{4} \sigma_{x} .
\end{array}
$$

The closure of a fundamental domain is $\left\{s(1,0)+t(0,1) \in \mathbb{R}^{2} \mid 0 \leq s, t \leq 1\right\}$. Then we find that the $D_{4}$-action on $T^{2}=\mathbb{R}^{2} / \Pi$ satisfies the assumptions in Lemma 4.4 , in which pt $=(0,0)$ and

$$
S^{1} \vee S^{1} \cong Y=((\mathbb{R} \oplus 0) /(\mathbb{Z} \oplus 0)) \vee((0 \oplus \mathbb{R}) /(0 \oplus \mathbb{Z}))
$$

To apply Lemma 4.5, we compute the cohomology of $Y$ :

Lemma 4.7. The equivariant cohomology of $Y$ is as follows:

\begin{tabular}{|c|c|c|c|c|}
\hline & $n=0$ & $n=1$ & $n=2$ & $n=3$ \\
\hline$H_{D_{4}}^{n}(Y ; \mathbb{Z})$ & $\mathbb{Z}$ & 0 & $\mathbb{Z}_{2}^{\oplus 3}$ & $\mathbb{Z}_{2}^{\oplus 2}$ \\
\hline
\end{tabular}

Proof. We use the Mayer-Vietoris exact sequence: Cover $Y$ by invariant subspaces $U$ and $V$ with the following $D_{4}$-equivariant homotopy equivalences

$$
U \simeq \mathrm{pt}, \quad V \simeq D_{4} / D_{2}^{(v)}, \quad U \cap V \simeq D_{4} / \mathbb{Z}_{2}^{(v)},
$$

where $D_{2}^{(v)}=\left\{1, C_{2}, \sigma_{x}, \sigma_{y}\right\} \cong D_{2}$ and $\mathbb{Z}_{2}^{(v)}=\left\{1, \sigma_{y}\right\} \cong \mathbb{Z}_{2}$. We can summarize the equivariant cohomology of these spaces in low degrees as follows:

\begin{tabular}{|l|c|c|c|}
\hline$n=3$ & & $\mathbb{Z}_{2} \oplus \mathbb{Z}_{2}$ & 0 \\
\hline$n=2$ & & $\mathbb{Z}_{2}^{\oplus 2} \oplus \mathbb{Z}_{2}^{\oplus 2}$ & $\mathbb{Z}_{2}$ \\
\hline$n=1$ & & 0 & 0 \\
\hline$n=0$ & & $\mathbb{Z} \oplus \mathbb{Z}$ & $\mathbb{Z}$ \\
\hline & $H_{D_{4}}^{n}(Y)$ & $H_{D_{4}}^{n}(U) \oplus H_{D_{4}}^{n}(V)$ & $H_{D_{4}}^{n}(U \cap V)$ \\
\hline
\end{tabular}

In the Mayer-Vietoris exact sequence

$$
\cdots \rightarrow H_{D_{4}}^{n}(Y) \rightarrow H_{D_{4}}^{n}(U) \oplus H_{D_{4}}^{n}(V) \stackrel{\Delta}{\rightarrow} H_{D_{4}}^{n}(U \cap V) \rightarrow H_{D_{4}}^{n+1}(Y) \rightarrow \cdots
$$


the map $\Delta: H_{D_{4}}^{n}(U) \oplus H_{D_{4}}^{n}(V) \rightarrow H_{D_{4}}^{n}(U \cap V)$ is expressed as $\Delta(u, v)=j_{U}^{*}(u)-j_{V}^{*}(v)$, where $j_{U}: U \cap V \rightarrow U$ and $j_{V}: U \cap V \rightarrow V$ are the inclusions. Under the natural identifications

$$
\begin{aligned}
& H_{D_{4}}^{2}(V) \cong H_{D_{2}^{(v)}}^{2}(\mathrm{pt}) \cong \operatorname{Hom}\left(D_{2}^{(v)}, U(1)\right), \\
& H_{D_{4}}^{2}(U \cap V) \cong H_{\mathbb{Z}_{2}^{(v)}}^{2}(\mathrm{pt}) \cong \operatorname{Hom}\left(\mathbb{Z}_{2}^{(v)}, U(1)\right),
\end{aligned}
$$

the map $j_{U}^{*}$ agrees with the homomorphism induced from the inclusion $\mathbb{Z}_{2}^{(v)} \rightarrow D_{2}^{(v)}$. This implies that $j_{U}^{*}$ is surjective, and so is $\Delta$ in degree 2. Clearly, $\Delta: H_{D_{4}}^{0}(U) \oplus H_{D_{4}}^{0}(V) \rightarrow H_{D_{4}}^{0}(U \cap V)$ is identified with the homomorphism $\mathbb{Z} \oplus \mathbb{Z} \rightarrow \mathbb{Z}$ given by $(m, n) \mapsto m-n$. Hence we can solve the Mayer-Vietoris exact sequence for $\{U, V\}$ to get the result claimed in this lemma.

Theorem $4.8(\mathrm{p} 4 \mathrm{~m} / \mathrm{p} 4 \mathrm{~g})$. The $D_{4}$-equivariant cohomology of $T^{2}$ in low degrees is as follows:

\begin{tabular}{|l|c|c|c|c|}
\hline & $n=0$ & $n=1$ & $n=2$ & $n=3$ \\
\hline$H_{D_{4}}^{n}\left(T^{2} ; \mathbb{Z}\right)$ & $\mathbb{Z}$ & 0 & $\mathbb{Z}_{2}^{\oplus 3}$ & $\mathbb{Z}_{2}^{\oplus 3}$ \\
\hline
\end{tabular}

We also have $F^{2} H_{D_{4}}^{3}\left(T^{2} ; \mathbb{Z}\right) \cong \mathbb{Z}_{2}^{\oplus 2}$.

Proof. In the Leray-Serre spectral sequence $E_{2}^{p, q}=H_{\text {group }}^{p}\left(D_{4} ; H^{q}\left(T^{2} ; \mathbb{Z}\right)\right)$, the $D_{4}$-modules $H^{0}\left(T^{2}\right), H^{1}\left(T^{2}\right)$ and $H^{2}\left(T^{2}\right)$ are identified with the trivial $D_{4}$-module $\mathbb{Z}, H^{1}(Y)$ and the $D_{4^{-}}$ module $\tilde{\mathbb{Z}}$ in Lemma 4.2 , respectively. Using Lemmas 4.1 and 4.2 , we can summarize the $E_{2}$-terms as follows:

\begin{tabular}{|c|c|c|c|c|}
\hline$q=3$ & 0 & 0 & 0 & 0 \\
\hline$q=2$ & 0 & $\mathbb{Z}_{2}$ & & \\
\hline$q=1$ & 0 & $\mathbb{Z}_{2}$ & $\mathbb{Z}_{2}$ & \\
\hline$q=0$ & $\mathbb{Z}$ & 0 & $\mathbb{Z}_{2}^{\oplus 2}$ & $\mathbb{Z}_{2}$ \\
\hline$E_{2}^{p, q}$ & $p=0$ & $p=1$ & $p=2$ & $p=3$ \\
\hline
\end{tabular}

Now the proof is completed by Lemma 4.5 .

\section{$4.5 \mathrm{p} 6 \mathrm{~m}$}

We let $\Pi=\mathbb{Z} a \oplus \mathbb{Z} b \subset \mathbb{R}^{2}$ be the lattice spanned by $a=\left(\begin{array}{l}1 \\ 0\end{array}\right)$ and $b=\left(\begin{array}{c}1 / 2 \\ \sqrt{3} / 2\end{array}\right)$. The point group $P$ is $D_{6}=\left\langle C, \sigma_{1} \mid C^{6}, \sigma_{1}^{2}, \sigma_{1} C \sigma_{1} C\right\rangle=\left\{1, C, C^{2}, C^{3}, C^{4}, C^{5}, \sigma_{1}, \sigma_{2}, \sigma_{3}, \sigma_{4}, \sigma_{5}, \sigma_{6}\right\}$, where $\sigma_{\ell}=C^{\ell-1} \sigma_{1}$. This group acts on $\Pi$ and $\mathbb{R}^{2}$ through the inclusion $D_{6} \subset \mathrm{O}(2)$ defined by

$$
C=\left(\begin{array}{cc}
1 / 2 & -\sqrt{3} / 2 \\
\sqrt{3} / 2 & 1 / 2
\end{array}\right), \quad \sigma_{1}=\left(\begin{array}{cc}
1 & 0 \\
0 & -1
\end{array}\right)
$$

If we use the identifications $a=1$ and $b=\tau=\exp 2 \pi i / 6$ under $\mathbb{R}^{2}=\mathbb{C}$, then the actions of $C \in$ $D_{6}$ and $\sigma_{1}$ are given by the multiplication by $\tau$ and the complex conjugation, respectively. The closure of a fundamental domain is $\{s a+t b \mid 0 \leq s, t \leq 1\}$ or equivalently $\{s+t \tau \mid 0 \leq s, t \leq 1\}$. We decompose this region to define a $D_{6}$-CW decomposition of $T^{2}$ as follows:

\begin{tabular}{|c|c|c|}
\hline 0 -cell & 1-cell & 2-cell \\
\hline$\tilde{e}_{0}^{0}=\mathrm{pt}$ & $\tilde{e}_{01}^{1}=\left(D_{6} /\left\{1, \sigma_{1}\right\}\right) \times e^{1}$ & $\tilde{e}^{2}=D_{6} \times e^{2}$ \\
$\tilde{e}_{1}^{0}=D^{6} /\left\{1, C^{3}, \sigma_{1}, \sigma_{4}\right\}$ & $\tilde{e}_{02}^{1}=\left(D_{6} /\left\{1, \sigma_{2}\right\}\right) \times e^{1}$ & \\
$\tilde{e}_{2}^{0}=D^{6} /\left\{1, C^{2}, C^{4}, \sigma_{2}, \sigma_{4}, \sigma_{6}\right\}$ & $\tilde{e}_{12}^{1}=\left(D_{6} /\left\{1, \sigma_{4}\right\}\right) \times e^{1}$ & \\
\hline
\end{tabular}


- $(0$-cell $)$ The 0 -cell $\tilde{e}_{0}^{0}=\left(D_{6} / D_{6}\right) \times e^{0}=\mathrm{pt}$ is the unique fixed point on $T^{2}$. The other 0 -cells are defined as follows:

$$
\begin{aligned}
& \tilde{e}_{1}^{0}=\left\{\frac{1}{2}, \frac{\tau}{2}, \frac{1+\tau}{2}\right\} \cong\left(D_{6} /\left\{1, C^{3}, \sigma_{1}, \sigma_{4}\right\}\right) \times e^{0} \\
& \tilde{e}_{2}^{0}=\left\{\frac{1+\tau}{3}, \frac{2(1+\tau)}{3}\right\} \cong\left(D_{6} /\left\{1, C^{2}, C^{4}, \sigma_{2}, \sigma_{4}, \sigma_{6}\right\}\right) \times e^{0}
\end{aligned}
$$

- (1-cell) For $0 \leq i<j \leq 2$, the 1-cell $\tilde{e}_{i j}^{1}$ consists of the six segments connecting $\tilde{e}_{i}^{0}$ and $\tilde{e}_{j}^{0}$. They are of the forms $\tilde{e}_{01}^{1}=\left(D_{6} /\left\{1, \sigma_{1}\right\}\right) \times e^{1}, \tilde{e}_{02}^{1}=\left(D_{6} /\left\{1, \sigma_{2}\right\}\right) \times e^{1}$ and $\tilde{e}_{12}^{1}=\left(D_{6} /\left\{1, \sigma_{4}\right\}\right) \times e^{1}$.
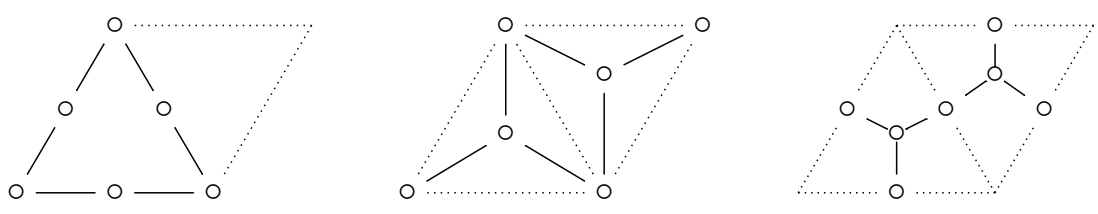

- (2-cell) The 2-cell $\tilde{e}^{2}=D_{6} \times e^{2}$ consists of the twelve small triangular regions surrounded by the 1-cells.

Let $Y \subset T^{2}$ be the invariant subspace $Y=\tilde{e}_{0}^{0} \cup \tilde{e}_{1}^{0} \cup \tilde{e}_{01}^{1}$.

Lemma 4.9. The equivariant cohomology of $Y$ is given as follows:

\begin{tabular}{|l|c|c|c|c|}
\hline & $n=0$ & $n=1$ & $n=2$ & $n=3$ \\
\hline$H_{D_{6}}^{n}(Y ; \mathbb{Z})$ & $\mathbb{Z}$ & 0 & $\mathbb{Z}_{2}^{\oplus 3}$ & $\mathbb{Z}_{2}^{\oplus 2}$ \\
\hline
\end{tabular}

Proof. We can find $D_{6}$-invariant subspaces $U$ and $V$ in $Y$ which have the following equivariant homotopy equivalences

$$
U \simeq \tilde{e}_{0}^{0}=\mathrm{pt}, \quad V \simeq \tilde{e}_{1}^{0}=D_{6} / D_{2}, \quad U \cap V \simeq \tilde{e}_{01}^{1} \simeq D_{6} / \mathbb{Z}_{2}^{(1)},
$$

where $D_{2}=\left\{1, C^{3}, \sigma_{1}, \sigma_{4}\right\}$ and $\mathbb{Z}_{2}^{(1)}=\left\{1, \sigma_{1}\right\}$. The equivariant cohomology groups of these spaces can be summarized as follows:

\begin{tabular}{|c|c|c|c|}
\hline$n=3$ & & $\mathbb{Z}_{2} \oplus \mathbb{Z}_{2}$ & 0 \\
\hline$n=2$ & & $\mathbb{Z}_{2}^{\oplus 2} \oplus \mathbb{Z}_{2}^{\oplus 2}$ & $\mathbb{Z}_{2}$ \\
\hline$n=1$ & & 0 & 0 \\
\hline$n=0$ & & $\mathbb{Z} \oplus \mathbb{Z}$ & $\mathbb{Z}$ \\
\hline & $H_{D_{6}}^{n}(Y)$ & $H_{D_{6}}^{n}(U) \oplus H_{D_{6}}^{n}(V)$ & $H_{D_{6}}^{n}(U \cap V)$ \\
\hline
\end{tabular}

In the Mayer-Vietoris exact sequence

$$
\cdots \rightarrow H_{D_{6}}^{n}(Y) \rightarrow H_{D_{6}}^{n}(U) \oplus H_{D_{6}}^{n}(V) \stackrel{\Delta}{\rightarrow} H_{D_{6}}^{n}(U \cap V) \rightarrow H_{D_{6}}^{n+1}(Y) \rightarrow \cdots
$$


the homomorphism $\Delta$ is expressed as $\Delta(u, v)=j_{U}^{*}(u)-j_{V}^{*}(v)$ with $j_{U}: U \cap V \rightarrow U$ and $j_{V}: U \cap V \rightarrow V$ the inclusions. This immediately determines $H_{D_{6}}^{0}(Y) \cong \mathbb{Z}$ and $H_{D_{6}}^{1}(Y)=0$. To complete the proof, we recall the identifications

$$
\begin{aligned}
& H_{D_{6}}^{2}(U) \cong \operatorname{Hom}\left(D_{6}, U(1)\right) \cong \mathbb{Z}_{2}^{\oplus 2}, \quad H_{D_{6}}^{2}(V) \cong \operatorname{Hom}\left(D_{2}, U(1)\right) \cong \mathbb{Z}_{2}^{\oplus 2}, \\
& H_{D_{6}}^{2}(U \cap V) \cong \operatorname{Hom}\left(\mathbb{Z}_{2}^{(1)}, U(1)\right) \cong \mathbb{Z}_{2},
\end{aligned}
$$

under which $j_{U}^{*}$ and $j_{V}^{*}$ are induced from the inclusions $D_{2} \rightarrow D_{6}$ and $\mathbb{Z}_{2}^{(1)} \rightarrow D_{6}$. As a basis of $H_{D_{6}}^{2}(U)$ we can choose the following 1-dimensional representations $\rho_{i}: D_{6} \rightarrow U(1)$ of $D_{6}$

$$
\rho_{1}:\left\{\begin{array}{l}
C \mapsto 1, \\
\sigma_{1} \mapsto-1,
\end{array} \quad \rho_{2}:\left\{\begin{array}{l}
C \mapsto-1, \\
\sigma_{1} \mapsto 1 .
\end{array}\right.\right.
$$

Similarly, we can choose the following 1-dimensional representations $\rho_{i}^{\prime}$ of $D_{2}=\left\{1, \sigma_{1}, C^{3}, \sigma_{4}\right\}$ as a basis of $H_{D_{6}}^{2}(V)$

$$
\rho_{1}^{\prime}:\left\{\begin{array}{l}
C^{3} \mapsto 1, \\
\sigma_{1} \mapsto-1,
\end{array} \quad \rho_{2}^{\prime}:\left\{\begin{array}{l}
C^{3} \mapsto-1, \\
\sigma_{1} \mapsto 1 .
\end{array}\right.\right.
$$

Now, we can see $H_{D_{6}}^{2}(Y) \cong \operatorname{Ker} \Delta \cong \mathbb{Z}_{2}^{\oplus 3}$, and it has the following basis

$$
\left\{\left(\rho_{1}, \rho_{1}^{\prime}\right),\left(\rho_{2}, \rho_{2}^{\prime}\right),\left(0, \rho_{2}^{\prime}\right)\right\} \subset \operatorname{Hom}\left(D_{6}, U(1)\right) \oplus \operatorname{Hom}\left(D_{4}, U(1)\right) .
$$

We can also see that $\Delta$ is surjective, and $H_{D_{6}}^{3}(Y) \cong \mathbb{Z}_{2}^{\oplus 2}$.

Let $X_{1}$ be the 1 -skeleton of the $D_{6}$-CW complex $T^{2}$.

Lemma 4.10. $H_{D_{6}}^{3}\left(X_{1} ; \mathbb{Z}\right) \cong \mathbb{Z}_{2}^{\oplus 2}$.

Proof. We cover $X_{1}=\tilde{e}_{0}^{0} \cup \tilde{e}_{1}^{0} \cup \tilde{e}_{2}^{0} \cup \tilde{e}_{01}^{1} \cup \tilde{e}_{02}^{1} \cup \tilde{e}_{12}^{1}$ by invariant subspaces $U^{\prime}$ and $V^{\prime}$ which admit the following equivariant homotopy equivalences

$$
U^{\prime} \simeq Y, \quad V^{\prime} \simeq \tilde{e}_{2}^{0}=D_{6} / D_{3}, \quad U^{\prime} \cap V^{\prime} \simeq \tilde{e}_{02}^{1} \sqcup \tilde{e}_{12}^{1} \simeq D_{6} / \mathbb{Z}_{2}^{(2)} \sqcup D_{6} / \mathbb{Z}_{2}^{(4)},
$$

where $D_{3}=\left\{1, C^{2}, C^{4}, \sigma_{2}, \sigma_{4}, \sigma_{6}\right\}, \mathbb{Z}_{2}^{(2)}=\left\{1, \sigma_{2}\right\}$ and $\mathbb{Z}_{2}^{(4)}=\left\{1, \sigma_{4}\right\}$. The equivariant cohomology groups of these spaces are summarized as follows:

\begin{tabular}{|c|c|c|c|}
\hline$n=3$ & & $\mathbb{Z}_{2}^{\oplus 2} \oplus 0$ & 0 \\
\hline$n=2$ & & $\mathbb{Z}_{2}^{\oplus 3} \oplus \mathbb{Z}_{2}$ & $\mathbb{Z}_{2} \oplus \mathbb{Z}_{2}$ \\
\hline$n=1$ & & 0 & 0 \\
\hline$n=0$ & & $\mathbb{Z} \oplus \mathbb{Z}$ & $\mathbb{Z} \oplus \mathbb{Z}$ \\
\hline & $H_{D_{6}}^{n}\left(X_{1}\right)$ & $H_{D_{6}}^{n}\left(U^{\prime}\right) \oplus H_{D_{6}}^{n}\left(V^{\prime}\right)$ & $H_{D_{6}}^{n}\left(U^{\prime} \cap V^{\prime}\right)$ \\
\hline
\end{tabular}

The homomorphism $\Delta$ in the Mayer-Vietoris exact sequence

$$
\cdots \rightarrow H_{D_{6}}^{2}\left(X_{1}\right) \rightarrow H_{D_{6}}^{2}\left(U^{\prime}\right) \oplus H_{D_{6}}^{2}\left(V^{\prime}\right) \stackrel{\Delta}{\rightarrow} H_{D_{6}}^{2}\left(U^{\prime} \cap V^{\prime}\right) \rightarrow H_{D_{6}}^{3}\left(X_{1}\right) \rightarrow \cdots
$$

is expressed as $\Delta(u, v)=j_{U^{\prime}}^{*}(u)-j_{V^{\prime}}^{*}(v)$ by using the inclusions $j_{U^{\prime}}: U^{\prime} \cap V^{\prime} \rightarrow U^{\prime}$ and $j_{V^{\prime}}: U^{\prime} \cap$ $V^{\prime} \rightarrow V^{\prime}$. An inspection proves that $j_{U^{\prime}}^{*}$ agrees with the composition of the following two homomorphisms: 
(i) the inclusion that follows from the calculation of $H_{D_{6}}^{2}(Y)$ in Lemma 4.9

$$
H_{D_{6}}^{2}\left(U^{\prime}\right) \cong H_{D_{6}}^{2}(Y) \longrightarrow \operatorname{Hom}\left(D_{6}, U(1)\right) \oplus \operatorname{Hom}\left(D_{2}, U(1)\right) .
$$

(ii) the direct sum $i_{2}^{*} \oplus i_{4}^{*}$ of the homomorphisms

$$
i_{2}^{*}: \operatorname{Hom}\left(D_{6}, U(1)\right) \rightarrow \operatorname{Hom}\left(\mathbb{Z}_{2}^{(2)}, U(1)\right), \quad i_{4}^{*}: \operatorname{Hom}\left(D_{2}, U(1)\right) \rightarrow \operatorname{Hom}\left(\mathbb{Z}_{2}^{(4)}, U(1)\right),
$$

induced from the inclusions $i_{2}: \mathbb{Z}_{2}^{(2)} \rightarrow D_{6}$ and $\mathbb{Z}_{2}^{(4)} \rightarrow D_{2}$.

Then, using the basis presented in the calculation of $H_{D_{6}}^{2}(Y)$, we find

$$
j_{U^{\prime}}^{*}\left(\rho_{1}, \rho_{1}^{\prime}\right)=(\rho, \rho), \quad j_{U^{\prime}}^{*}\left(\rho_{2}, \rho_{2}^{\prime}\right)=(\rho, \rho), \quad j_{U^{\prime}}^{*}\left(0, \rho_{2}^{\prime}\right)=(0, \rho),
$$

where $\rho: \mathbb{Z}_{2} \rightarrow \mathbb{Z}_{2}$ is the identity map generating $\operatorname{Hom}\left(\mathbb{Z}_{2}, U(1)\right) \cong \mathbb{Z}_{2}$. Hence $j_{U^{\prime}}^{*}$ as well as $\Delta$ are surjective, and $H_{D_{6}}^{3}\left(X_{1}\right) \cong H_{D_{6}}^{3}(Y) \cong \mathbb{Z}_{2}^{\oplus 2}$.

Theorem $4.11(\mathrm{p} 6 \mathrm{~m}) \cdot H_{D_{6}}^{3}\left(T^{2} ; \mathbb{Z}\right) \cong \mathbb{Z}_{2}^{\oplus 2}$.

Proof. The relevant part of the exact sequence for the pair $\left(T^{2}, X_{1}\right)$ is

$$
H_{D_{6}}^{3}\left(T^{2}, X_{1}\right) \rightarrow H_{D_{6}}^{3}\left(T^{2}\right) \rightarrow H_{D_{6}}^{3}\left(X_{1}\right) \rightarrow H_{D_{6}}^{4}\left(T^{2}, X_{1}\right) .
$$

By means of the excision axiom, we have $H_{D_{6}}^{n}\left(T^{2}, X_{1}\right) \cong H^{n-2}(\mathrm{pt})$. Therefore we get $H_{D_{6}}^{3}\left(T^{2}\right) \cong$ $H_{D_{6}}^{3}\left(X_{1}\right) \cong \mathbb{Z}_{2}^{\oplus 2}$.

Let $\hat{\mathbb{Z}}=\mathbb{Z}_{\phi_{1}}$ be the $D_{6}$-module such that its underlying group is $\mathbb{Z}$ and $D_{6}$ acts via the homomorphism $\phi_{1}: D_{6} \rightarrow \mathbb{Z}_{2}$ given by $\phi_{1}(C)=-1$ and $\phi_{1}\left(\sigma_{1}\right)=1$.

Lemma 4.12. There is an exact sequence of $D_{6}$-modules

$$
0 \rightarrow H^{1}\left(T^{2} ; \mathbb{Z}\right) \rightarrow H^{1}(Y ; \mathbb{Z}) \stackrel{\pi}{\rightarrow} \hat{\mathbb{Z}} \rightarrow 0
$$

admitting a module homomorphism $s: \hat{\mathbb{Z}} \rightarrow H^{1}(Y ; \mathbb{Z})$ such that $\pi \circ s=3$.

Proof. Let $\eta_{1}, \eta_{2} \in H_{1}\left(T^{2}\right)$ be the homology classes of the loops going along the vectors 1 and $\tau$ respectively in the fundamental domain, which form a basis of $H_{1}\left(T^{2}\right) \cong \mathbb{Z}^{2}$. Also, let $\gamma_{1}, \gamma_{2}, \gamma_{3} \in H_{1}(Y)$ be the homology classes of loops along 1, $\tau$ and $\tau-1$, which form a basis of $H_{1}(Y) \cong \mathbb{Z}^{3}$. The inclusion map $i: Y \rightarrow T^{2}$ relates these bases by $i_{*} \gamma_{1}=\eta_{1}, i_{*} \gamma_{2}=\eta_{2}$ and $i_{*} \gamma_{3}=\eta_{2}-\eta_{1}$. The actions of $C \in D_{6}$ and $\sigma_{1}$ on these bases are

$$
\left\{\begin{array} { l } 
{ C _ { * } \eta _ { 1 } = \eta _ { 2 } , } \\
{ C _ { * } \eta _ { 2 } = \eta _ { 2 } - \eta _ { 1 } , }
\end{array} \quad \left\{\begin{array} { l } 
{ \sigma _ { 1 * } \eta _ { 1 } = \eta _ { 1 } , } \\
{ \sigma _ { 1 * } \eta _ { 2 } = \eta _ { 1 } - \eta _ { 2 } , }
\end{array} \quad \left\{\begin{array} { l } 
{ C _ { * } \gamma _ { 1 } = \gamma _ { 2 } , } \\
{ C _ { * } \gamma _ { 2 } = \gamma _ { 3 } , } \\
{ C _ { * } \gamma _ { 3 } = - \gamma _ { 1 } , }
\end{array} \quad \left\{\begin{array}{l}
\sigma_{1 *} \gamma_{1}=\gamma_{1}, \\
\sigma_{1 *} \gamma_{2}=-\gamma_{3}, \\
\sigma_{1 *} \gamma_{3}=-\gamma_{2} .
\end{array}\right.\right.\right.\right.
$$

Let $\left\{h_{1}, h_{2}\right\} \subset H^{1}\left(T^{2}\right)$ and $\left\{g_{1}, g_{2}, g_{3}\right\} \subset H^{1}(Y)$ be dual to the homology bases. They are related by $i^{*} h_{1}=g_{1}-g_{3}$ and $i^{*} h_{2}=g_{2}+g_{3}$, and the induced $D_{6}$-actions are as follows.

$$
\left\{\begin{array} { l } 
{ C ^ { * } h _ { 1 } = - h _ { 2 } , } \\
{ C ^ { * } h _ { 2 } = h _ { 1 } + h _ { 2 } , }
\end{array} \quad \left\{\begin{array} { l } 
{ \sigma _ { 1 } ^ { * } h _ { 1 } = h _ { 1 } + h _ { 2 } , } \\
{ \sigma _ { 1 } ^ { * } h _ { 2 } = - h _ { 2 } , }
\end{array} \quad \left\{\begin{array} { l } 
{ C ^ { * } g _ { 1 } = - g _ { 3 } , } \\
{ C ^ { * } g _ { 2 } = g _ { 1 } , } \\
{ C ^ { * } g _ { 3 } = g _ { 2 } , }
\end{array} \quad \left\{\begin{array}{l}
\sigma_{1}^{*} g_{1}=g_{1}, \\
\sigma_{1}^{*} g_{2}=-g_{3}, \\
\sigma_{1}^{*} g_{3}=-g_{2} .
\end{array}\right.\right.\right.\right.
$$

These expressions allow us to prove that the cokernel of the homomorphism $i^{*}: H^{1}\left(T^{2}\right) \rightarrow H^{2}(Y)$ is isomorphic to $\hat{\mathbb{Z}}$, yielding the exact sequence. The homomorphism $s: \hat{\mathbb{Z}} \rightarrow H^{1}(Y)$ is given by $s(1)=g_{1}-g_{2}+g_{3}$. 
Lemma 4.13. $H_{\text {group }}^{n}\left(D_{6} ; H^{1}\left(T^{2} ; \mathbb{Z}\right)\right)=0$ for $n=0,1,2$.

Proof. We use the long exact sequence in group cohomology induced from the exact sequence $0 \rightarrow H^{1}\left(T^{2}\right) \rightarrow H^{1}(Y) \stackrel{\pi}{\rightarrow} \hat{\mathbb{Z}} \rightarrow 0$ in coefficients. By Lemmas 4.1, 4.9 and 5.6 to be given in Section 5 , we get the following:

\begin{tabular}{|c|c|c|c|}
\hline$n=2$ & & $\mathbb{Z}_{2}$ & $\mathbb{Z}_{2}$ \\
\hline$n=1$ & & $\mathbb{Z}_{2}$ & $\mathbb{Z}_{2}$ \\
\hline$n=0$ & & 0 & 0 \\
\hline & $H_{\text {group }}^{n}\left(D_{6} ; H^{1}\left(T^{2}\right)\right)$ & $H_{\text {group }}^{n}\left(D_{6} ; H^{1}(Y)\right)$ & $H_{\text {group }}^{n}\left(D_{6} ; \hat{\mathbb{Z}}\right)$ \\
\hline
\end{tabular}

It is clear that $H_{\text {group }}^{0}\left(D_{6} ; H^{1}\left(T^{2}\right)\right)=0$. The homomorphism in group cohomology induced from $\pi: H^{1}(Y) \rightarrow \hat{\mathbb{Z}}$ is surjective in degree 1 and 2 , because $\pi \circ s=3$. This leads to the remaining vanishing.

Theorem 4.14 (p6m). The following holds true:

(a) $F^{2} H_{D_{6}}^{3}\left(T^{2} ; \mathbb{Z}\right) \cong \mathbb{Z}_{2}$,

(b) the $D_{6}$-equivariant cohomology of $T^{2}$ in low degrees is as follows:

$$
H_{D_{6}}^{0}\left(T^{2} ; \mathbb{Z}\right) \cong \mathbb{Z}, \quad H_{D_{6}}^{1}\left(T^{2} ; \mathbb{Z}\right)=0, \quad H_{D_{6}}^{2}\left(T^{2} ; \mathbb{Z}\right) \cong \mathbb{Z}_{2}^{\oplus 2} .
$$

Proof. In the $E_{2}$-term of the Leray-Serre spectral sequence $E_{2}^{p, q}=H_{\text {group }}^{p}\left(D_{6} ; H^{q}\left(T^{2} ; \mathbb{Z}\right)\right)$, the coefficient $H^{0}\left(T^{2}\right)$ is identified with the trivial $D_{6}$-module $\mathbb{Z}$, and $H^{2}\left(T^{2}\right)$ with $\tilde{\mathbb{Z}}$ as in Lemma 4.2. The group cohomology with coefficients in $H^{1}\left(T^{2}\right)$ is already computed, and that in $\tilde{\mathbb{Z}}$ is also computed in Lemma 4.2 . The $E_{2}$-terms are summarized as follows:

\begin{tabular}{|c|c|c|c|c|}
\hline$q=3$ & 0 & 0 & 0 & 0 \\
\hline$q=2$ & 0 & $\mathbb{Z}_{2}$ & & \\
\hline$q=1$ & 0 & 0 & 0 & \\
\hline$q=0$ & $\mathbb{Z}$ & 0 & $\mathbb{Z}_{2}^{\oplus 2}$ & $\mathbb{Z}_{2}$ \\
\hline$E_{2}^{p, q}$ & $p=0$ & $p=1$ & $p=2$ & $p=3$ \\
\hline
\end{tabular}

This list and Lemma 4.3 lead to the theorem.

\subsection{The proof of Corollary 1.2}

The only non-trivial point in the corollary is (c), which we prove here. Let $P$ be the point group of one of the 2-dimensional space groups. We can assume that $P$ does not preserve the orientation of $T^{2}$. Then we have

$$
F^{2} H_{P}^{3}\left(T^{2} ; \mathbb{Z}\right) \cong E_{2}^{2,1} \oplus E_{2}^{3,0}
$$

by Lemma 4.3, in which the direct summands are

$$
E_{2}^{2,1}=H_{\text {group }}^{2}\left(P ; H^{1}\left(T^{2} ; \mathbb{Z}\right)\right), \quad E_{2}^{3,0}=H_{\text {group }}^{3}(P ; \mathbb{Z}) \cong H_{\text {group }}^{2}(P ; U(1)) .
$$

Thus, it suffices to prove that the group cocycles $\tau$ induced from the nonsymmorphic 2dimensional space groups as in Section 2 generate $E_{2}^{2,1}$.

Recall from Section 2 that the group 2-cocycle $\nu \in Z_{\text {group }}^{2}(P ; \Pi)$ measures the failure for a space group $S$ to be a semi-direct product of its point group $P$ and the lattice $\Pi$, where $\Pi$ is regarded as a left $P$-module naturally. In other words, $S$ is nonsymmorphic if and only if $[\nu] \in H_{\text {group }}^{2}(P ; \Pi)$ is non-trivial. 
Lemma 4.15. Let $\Pi$ and $P$ be the lattice and the point group of a d-dimensional space group $S$. Then there is an isomorphism of groups

$$
H_{\text {group }}^{2}(P ; \Pi) \cong H_{\text {group }}^{2}\left(P ; H^{1}(\hat{\Pi} ; \mathbb{Z})\right) .
$$

In particular, this factors through the homomorphisms

$$
H_{\text {group }}^{2}(P ; \Pi) \longrightarrow H_{\text {group }}^{2}(P ; C(\hat{\Pi}, U(1)))
$$

given by the assignment of the cocycles $\nu \mapsto \tau$ in Section 2 and

$$
H_{\text {group }}^{2}(P ; C(\hat{\Pi}, U(1))) \longrightarrow H_{\text {group }}^{2}\left(P ; H^{1}(\hat{\Pi} ; \mathbb{Z})\right)
$$

induced from the natural surjection $\delta: C(\hat{\Pi}, U(1)) \rightarrow H^{1}(\hat{\Pi} ; \mathbb{Z})$.

Proof. Instead of the left $P$-action on the Pontryagin dual $\hat{\Pi}=\operatorname{Hom}(\Pi, U(1))$ defined in Section 2, we consider the natural right action $\hat{k}(m) \mapsto \hat{k}(p m)$ of $p \in P$ on $\hat{k} \in \hat{\Pi}$, from which the left action originates. This choice of the actions does not affect the group cohomology. The right $P$-action on $\hat{\Pi}$ induces by pull-back a left $P$-action on the cohomology $H^{1}(\hat{\Pi} ; \mathbb{Z})$. Thus, the isomorphism of the group cohomologies will be established once we see $H^{1}(\hat{\Pi} ; \mathbb{Z}) \cong \Pi$ as left $P$-modules. In general, for each element $m \in \Pi \subset \mathbb{R}^{d}=V$, the path $[0,1] \rightarrow V,(t \mapsto t m)$ defines a loop in $V / \Pi$. This induces an isomorphism of left $P$-modules $\Pi \cong H_{1}(V / \Pi ; \mathbb{Z})$. By the universal coefficient theorem, the dual $\Pi^{*}=\operatorname{Hom}(\Pi, \mathbb{Z})$ of $\Pi$ is identified with the first homology group of $V / \Pi$ as a right $P$-module:

$$
\Pi^{*}=\operatorname{Hom}(\Pi, \mathbb{Z}) \cong \operatorname{Hom}\left(H_{1}(V / \Pi ; \mathbb{Z}), \mathbb{Z}\right) \cong H^{1}(V / \Pi ; \mathbb{Z}) .
$$

Considering the dual space $V^{*}=\operatorname{Hom}(V, \mathbb{R})$ and its lattice $\Pi^{*}$ instead, we similarly get an isomorphism of left $P$-modules

$$
\Pi \cong H^{1}\left(V^{*} / \Pi^{*} ; \mathbb{Z}\right) .
$$

Since there is a natural isomorphism of tori $V^{*} / \Pi^{*} \rightarrow \hat{\Pi}=\operatorname{Hom}(\Pi, U(1))$ with right $P$-actions, the isomorphism of the group cohomologies is proved. The factoring of the isomorphism can be verified by a direct inspection.

Now, in the case of $\mathrm{pm} / \mathrm{pg}$, the nonsymmorphic group pg defines the non-trivial element of $H_{\text {group }}^{2}\left(\mathbb{Z}_{2} ; \Pi\right) \cong \mathbb{Z}_{2}$ through $\nu$, and the element corresponds by Lemma 4.15 to the nontrivial element of $H_{\text {group }}^{2}\left(\mathbb{Z}_{2} ; H^{1}\left(T^{2} ; \mathbb{Z}\right)\right) \cong \mathbb{Z}_{2}$ represented by the group 2-cocycle $\tau$ induced from $\mathrm{pg}$. The same holds true in the case of $\mathrm{p} 4 \mathrm{~m} / \mathrm{p} 4 \mathrm{~g}$. In the case of $\mathrm{pmm} / \mathrm{pmg} / \mathrm{pgg}$, we have $H_{\text {group }}^{2}\left(D_{2} ; \Pi\right) \cong H_{\text {group }}^{2}\left(D_{2} ; H^{1}\left(T^{2} ; \mathbb{Z}\right)\right) \cong \mathbb{Z}_{2} \oplus \mathbb{Z}_{2}$. In view of the classification of 2dimensional space groups ([12]), the non-trivial elements $(-1,1)$ and $(-1,-1)$, with respect to a basis of $\mathbb{Z}_{2} \oplus \mathbb{Z}_{2}$, correspond to the nonsymmorphic groups pmg and pgg respectively. (The nonsymmorphic group corresponding to $(1,-1)$ is equivalent to pmg through an affine transformation preserving the lattice.) Therefore $H_{\text {group }}^{2}\left(D_{2} ; H^{1}\left(T^{2} ; \mathbb{Z}\right)\right) \cong \mathbb{Z}_{2} \oplus \mathbb{Z}_{2}$ is generated by the group 2-cocycles induced from the nonsymmorphic groups.

\section{The twisted case}

This section concerns the equivariant cohomology with local coefficients. We start with some remarks about the Leray-Serre spectral sequence, focusing on the similarities and the differences with the case of the usual Borel equivariant cohomology. We then summarize tools for computation in the version adapted to the case with local coefficients. After that, we prove Theorems 1.5 and 1.6: As in the untwisted case, the full computation is lengthy, and the details are only provided in the case of $\mathrm{p} 6 \mathrm{~m}$. 


\subsection{The Leray-Serre spectral sequence}

For a finite group $G$ and a homomorphism $\phi: G \rightarrow \mathbb{Z}_{2}$, we define $\mathbb{Z}_{\phi}$ to be the $G$-module $\mathbb{Z}_{\phi}$ such that its underlying group is $\mathbb{Z}$ and $G$ acts via $\phi$. Suppose that $G$ acts on a space $X$. Associated to the fibration $X \rightarrow E G \times_{G} X \rightarrow B G$ is the long exact sequence of homotopy groups:

$$
\cdots \rightarrow \pi_{n}(X) \rightarrow \pi_{n}\left(E G \times_{G} X\right) \rightarrow \pi_{n}(B G) \rightarrow \cdots
$$

Since $\pi_{1}(B G) \cong G$, we get a homomorphism $\pi_{1}\left(E G \times_{G} X\right) \rightarrow \mathbb{Z}_{2}$ by composing $\pi_{1}\left(E G \times{ }_{G} X\right) \rightarrow G$ with $\phi: G \rightarrow \mathbb{Z}_{2}$. The homomorphism defines a local system on the Borel construction $E G \times{ }_{G} X$, which we denote with the same notation $\mathbb{Z}_{\phi}$. By using this local system, we define the $G$ equivariant cohomology with local coefficients

$$
H_{G}^{n}\left(X ; \mathbb{Z}_{\phi}\right)=H^{n}\left(E G \times_{G} X ; \mathbb{Z}_{\phi}\right)
$$

Of course, if $\phi$ is trivial, then the cohomology above recovers the usual equivariant cohomology with integer coefficients $\mathbb{Z}$.

For $H_{G}^{n}\left(X ; \mathbb{Z}_{\phi}\right)$, we also have the Leray-Serre spectral sequence $E_{r}^{p, q}$ converging to the graded quotient of a filtration

$$
H_{G}^{n}\left(X ; \mathbb{Z}_{\phi}\right)=F^{0} H_{G}^{n}\left(X ; \mathbb{Z}_{\phi}\right) \supset F^{1} H_{G}^{n}\left(X ; \mathbb{Z}_{\phi}\right) \supset F^{2} H_{G}^{n}\left(X ; \mathbb{Z}_{\phi}\right) \supset \cdots
$$

Its $E_{2}$-term is again given by the group cohomology

$$
E_{2}^{p, q}=H_{\text {group }}^{p}\left(G ; H^{q}(X ; \mathbb{Z}) \otimes \mathbb{Z}_{\phi}\right),
$$

where $H^{q}(X ; \mathbb{Z}) \otimes \mathbb{Z}_{\phi}$ is the tensor product of the $G$-modules $H^{q}(X ; \mathbb{Z})$ and $\mathbb{Z}_{\phi}$, namely, its underlying group is

$$
H^{q}(X ; \mathbb{Z}) \otimes \mathbb{Z}_{\phi}=H^{q}(X ; \mathbb{Z}) \otimes \mathbb{Z} \cong H^{q}(X ; \mathbb{Z}),
$$

and $g \in G$ acts on $x \in H^{q}(X ; \mathbb{Z})$ by $x \mapsto \phi(g) \cdot g^{*} x$.

As in the usual equivariant cohomology, $H_{G}^{n}\left(X ; \mathbb{Z}_{\phi}\right)$ can be identified with a sheaf cohomology of the simplicial space $G^{\bullet} \times X$. (See for instance the appendix of [10] in the case of $G=\mathbb{Z}_{2}$.) This interpretation leads to the classification of the twists for the Freed-Moore $K$-theory [8], whose definition is similar to the one given in Section 3.2 (see [18]). In terms of the Borel equivariant cohomology, the graded twists are classified by $H_{G}^{1}\left(X ; \mathbb{Z}_{2}\right) \times H_{G}^{3}\left(X ; \mathbb{Z}_{\phi}\right)$ and the ungraded twists by $H_{G}^{3}\left(X ; \mathbb{Z}_{\phi}\right)$.

The results in Section 3.3 can be generalized to the equivariant cohomology with coefficients in $\mathbb{Z}_{\phi}$, and we get the following geometric interpretation generalizing Corollary 3.12:

Proposition 5.1. Let $G$ be a finite group acting on a compact and path connected space $X$ with at least one fixed point, and $\phi: G \rightarrow \mathbb{Z}_{2}$ a homomorphism.

(i) $F^{1} H_{G}^{3}\left(X ; \mathbb{Z}_{\phi}\right)$ classifies (ungraded) twists which can be represented by $\phi$-twisted central extensions of the groupoid $X / / G$.

(ii) $F^{2} H_{G}^{3}\left(X ; \mathbb{Z}_{\phi}\right)$ classifies (ungraded) twists which can be represented by 2 -cocycles of $G$ with coefficients in the $G$-module $C(X, U(1))_{\phi}$, where $C(X, U(1))_{\phi}=C(X, U(1))$ is the group of $U(1)$-valued functions on $X$ and $g \in G$ acts on $f: X \rightarrow U(1)$ by $f \mapsto g^{*} f^{\phi(g)}$.

(iii) $F^{3} H_{G}^{3}\left(X ; \mathbb{Z}_{\phi}\right)=H_{\text {group }}^{2}\left(G ; U(1)_{\phi}\right)$ classifies (ungraded) twists which can be represented by 2 -cocycles of $G$ with coefficients in the $G$-module $U(1)_{\phi}$, where $U(1)=U(1)_{\phi}$ as a group and $g \in G$ acts on $u \in U(1)$ by $u \mapsto u^{\phi(g)}$. 


\subsection{Tools}

As long as we are concerned with the local system $\mathbb{Z}_{\phi}$ associated to a homomorphism $\phi: G \rightarrow \mathbb{Z}_{2}$, the reduced cohomology $\tilde{H}_{G}^{n}\left(X ; \mathbb{Z}_{\phi}\right)$ makes sense for a $G$-space $X$ with a fixed point pt $\in X$, and we have the direct sum decomposition $H_{G}^{n}\left(X ; \mathbb{Z}_{\phi}\right) \cong H_{G}^{n}\left(\mathrm{pt} ; \mathbb{Z}_{\phi}\right) \oplus \tilde{H}_{G}^{n}\left(X ; \mathbb{Z}_{\phi}\right)$. Then we can generalize the proof of Lemma 4.1 to show:

Lemma 5.2. Suppose that a finite group $G$ acts on a path connected space $Y$ fixing at least one point $\mathrm{pt} \in Y$. Suppose further that $Y$ is a $C W$ complex consisting of only cells of dimension less than or equal to 1 . Then, for any homomorphism $\phi: G \rightarrow \mathbb{Z}_{2}$ and $n \geq 0$, the following holds true:

$$
H_{\text {group }}^{n}\left(G ; H^{1}(Y ; \mathbb{Z}) \otimes \mathbb{Z}_{\phi}\right) \cong \tilde{H}_{G}^{n+1}\left(Y ; \mathbb{Z}_{\phi}\right) .
$$

Similarly, we can generalize Lemma 4.5 as follows:

Lemma 5.3. Suppose a finite group $G$ acts on the torus $T^{2}=S^{1} \times S^{1}$ and

- there is a fixed point $\mathrm{pt}=\left(x_{0}, y_{0}\right) \in T^{2}$ under the $G$-action,

- $G$ preserves the subspace $S^{1} \vee S^{1}=S^{1} \times\left\{y_{0}\right\} \cup\left\{x_{0}\right\} \times S^{1} \subset T^{2}$.

Then there is the following isomorphism of groups for any homomorphism $\phi: G \rightarrow \mathbb{Z}_{2}$ and all $n \in \mathbb{Z}$

$$
H_{G}^{n}\left(T^{2} ; \mathbb{Z}_{\phi}\right) \cong H_{G}^{n}\left(\mathrm{pt} ; \mathbb{Z}_{\phi}\right) \oplus \tilde{H}_{G}^{n}\left(S^{1} \vee S^{1} ; \mathbb{Z}_{\phi}\right) \oplus \tilde{H}_{G}^{n}\left(T^{2} / S^{1} \vee S^{1} ; \mathbb{Z}_{\phi}\right)
$$

Further, the Leray-Serre spectral sequence for $H_{G}^{n}\left(T^{2} ; \mathbb{Z}_{\phi}\right)$ degenerates at $E_{2}$ and the relevant extension problems are trivial, so that

(a) $F^{2} H_{G}^{3}\left(T^{2} ; \mathbb{Z}_{\phi}\right) \cong E_{2}^{2,1} \oplus E_{2}^{3,0}$,

(b) $H_{G}^{n}\left(T^{2} ; \mathbb{Z}_{\phi}\right) \cong \bigoplus_{p+q=n} E_{2}^{p, q}$ for all $n \in \mathbb{Z}$.

Besides the generalizations above, we will use the following universal coefficient theorem in the sequel:

Lemma 5.4. For any $\phi: G \rightarrow \mathbb{Z}_{2}$, there is a split exact sequence of groups

$$
0 \rightarrow H_{G}^{n}\left(X ; \mathbb{Z}_{\phi}\right) \otimes \mathbb{Z}_{2} \rightarrow H_{G}^{n}\left(X ; \mathbb{Z}_{2}\right) \rightarrow \operatorname{Tor}\left(H_{G}^{n+1}\left(X ; \mathbb{Z}_{\phi}\right), \mathbb{Z}_{2}\right) \rightarrow 0 .
$$

Proof. For any homomorphism $\phi: G \rightarrow \mathbb{Z}_{2}$, let $\left(\mathbb{Z}_{2}\right)_{\phi}$ be the $G$-module such that its underlying group is $\mathbb{Z}_{2}$ and its $G$-action is given by $\phi: G \rightarrow \mathbb{Z}_{2}$. This $G$-module $\left(\mathbb{Z}_{2}\right)_{\phi}$ agrees with the trivial $G$-module $\mathbb{Z}_{2}$, even if $\phi$ is non-trivial. Then, looking at the cochain complexes defining the equivariant cohomology, the usual proof of the universal coefficient theorem leads to the lemma. Another proof is to use the Thom isomorphism, which unwinds the local coefficients: Let $\underline{\mathbb{R}}_{\phi} \rightarrow X$ be the $G$-equivariant real line bundle on $X$ whose underlying bundle is $X \times \mathbb{R}$ and the action of $g \in G$ on $(x, r) \in X \times \mathbb{R}$ is $(x, r) \mapsto(g x, \phi(g) r)$. The Thom isomorphism theorem then provides

$$
H_{G}^{n}\left(X ; A_{\phi}\right) \cong H_{G}^{n+1}(D, S ; A),
$$

where $A$ is $\mathbb{Z}_{2}$ or $\mathbb{Z}$, and $D \subset \underline{\mathbb{R}}_{\phi}$ and $S \subset \underline{\mathbb{R}}_{\phi}$ are the unit disk bundle and the unit sphere bundle, respectively. Then the usual universal coefficient theorem leads to the present lemma.

To compute the equivariant cohomology $H_{G}^{n}\left(X ; \mathbb{Z}_{\phi}\right)$, we usually need the cohomology of the point $H_{G}^{n}\left(\mathrm{pt} ; \mathbb{Z}_{\phi}\right)$. This cohomology is identified with the group cohomology $H_{\text {group }}^{n}\left(G ; \mathbb{Z}_{\phi}\right)$ by the degeneration of the Leray-Serre spectral sequence, but its direct computation is not realistic except for the simplest cases (cf. Lemma 4.2). A useful way to compute it is: 
Lemma 5.5. For any $\phi: G \rightarrow \mathbb{Z}_{2}$, there are natural exact sequences

$$
\begin{aligned}
& \cdots \rightarrow H_{G}^{n-1}\left(\mathrm{pt} ; \mathbb{Z}_{\phi}\right) \rightarrow H_{G}^{n}(\mathrm{pt} ; \mathbb{Z}) \stackrel{i^{*}}{\rightarrow} H_{\mathrm{Ker} \phi}^{n}(\mathrm{pt} ; \mathbb{Z}) \rightarrow H_{G}^{n}\left(\mathrm{pt} ; \mathbb{Z}_{\phi}\right) \rightarrow \cdots, \\
& \cdots \rightarrow H_{G}^{n-1}(\mathrm{pt} ; \mathbb{Z}) \rightarrow H_{G}^{n}\left(\mathrm{pt} ; \mathbb{Z}_{\phi}\right) \stackrel{i^{*}}{\rightarrow} H_{\mathrm{Ker} \phi}^{n}(\mathrm{pt} ; \mathbb{Z}) \rightarrow H_{G}^{n}(\mathrm{pt} ; \mathbb{Z}) \rightarrow \cdots,
\end{aligned}
$$

where $i^{*}$ is induced from the inclusion $i: \operatorname{Ker} \phi \rightarrow G$.

Proof. Let $G$ act on $\mathbb{R}_{\phi}=\mathbb{R}$ via $\phi: G \rightarrow \mathbb{Z}_{2}$. We can regard $\mathbb{R}_{\phi}$ as a $G$-equivariant real line bundle on pt. We have the Thom isomorphisms

$$
H_{G}^{n}\left(\mathrm{pt} ; \mathbb{Z}_{\phi}\right) \cong H_{G}^{n+1}(D, S ; \mathbb{Z}), \quad H_{G}^{n}(\mathrm{pt} ; \mathbb{Z}) \cong H_{G}^{n+1}\left(D, S ; \mathbb{Z}_{\phi}\right),
$$

where $D$ and $S$ are the unit interval in $\mathbb{R}_{\phi}$ and its boundary, respectively. Note that $D$ is equivariantly contractible. Note also that $S \cong G / \operatorname{Ker} \phi$ as a $G$-space. Thus, we have isomorphisms

$$
H_{G}^{n}\left(D ; \mathbb{Z}_{\phi}\right) \cong H_{G}^{n}\left(\mathrm{pt} ; \mathbb{Z}_{\phi}\right), \quad H_{G}^{n}\left(S ; \mathbb{Z}_{\phi}\right) \cong H_{\operatorname{Ker} \phi}^{n}(\mathrm{pt} ; \mathbb{Z}) .
$$

Substituting these isomorphisms and the Thom isomorphisms into the exact sequences for the pair $(D, S)$, we complete the proof.

By means of the lemma above, we get:

Lemma 5.6. Let $P$ be $\mathbb{Z}_{2 m}, D_{2 m-1}$ or $D_{2 m}$ with $m \geq 1$. The P-equivariant cohomology of the point with coefficients in $\mathbb{Z}_{\phi}$,

$$
H_{P}^{n}\left(\mathrm{pt} ; \mathbb{Z}_{\phi}\right) \cong H_{\text {group }}^{n}\left(P ; \mathbb{Z}_{\phi}\right)
$$

in low degrees is given as follows:

\begin{tabular}{|c|c|c|c|c|c|}
\hline$P$ & $\phi$ & $H_{P}^{0}\left(\mathrm{pt} ; \mathbb{Z}_{\phi}\right)$ & $H_{P}^{1}\left(\mathrm{pt} ; \mathbb{Z}_{\phi}\right)$ & $H_{P}^{2}\left(\mathrm{pt} ; \mathbb{Z}_{\phi}\right)$ & $H_{P}^{3}\left(\mathrm{pt} ; \mathbb{Z}_{\phi}\right)$ \\
\hline $\mathbb{Z}_{2 m}$ & $\phi_{1}$ & 0 & $\mathbb{Z}_{2}$ & 0 & $\mathbb{Z}_{2}$ \\
\hline$D_{2 m-1}$ & $\phi_{0}$ & 0 & $\mathbb{Z}_{2}$ & $\mathbb{Z}_{2 m-1}$ & $\mathbb{Z}_{2}$ \\
\hline$D_{2 m}$ & $\phi_{0}$ & 0 & $\mathbb{Z}_{2}$ & $\mathbb{Z}_{2 m}$ & $\mathbb{Z}_{2}^{\oplus 2}$ \\
\hline$D_{2 m}$ & $\phi_{1}, \phi_{2}$ & 0 & $\mathbb{Z}_{2}$ & $\mathbb{Z}_{2}$ & $\mathbb{Z}_{2}^{\oplus 2}$ \\
\hline
\end{tabular}

Proof. In the case of $D_{2 m}$ with $m$ even and $\phi \neq \phi_{0}$, the first exact sequence in Lemma 5.5 leads to $H_{D_{2 m}}^{0}\left(\mathrm{pt} ; \mathbb{Z}_{\phi}\right)=0$ and $H_{D_{2 m}}^{1}\left(\mathrm{pt} ; \mathbb{Z}_{\phi}\right) \cong \mathbb{Z}_{2}$. This computation also shows that $H_{D_{2 m}}^{2}$ (pt; $\left.\mathbb{Z}_{\phi}\right)$ contains $\mathbb{Z}_{2}$ as a subgroup. Here, applying the universal coefficient theorem to $H_{P}^{n}(\mathrm{pt} ; \mathbb{Z})$, we compute the cohomology with coefficients in $\mathbb{Z}_{2}$ to have $H_{D_{2 m}}^{1}\left(\mathrm{pt} ; \mathbb{Z}_{2}\right) \cong \mathbb{Z}_{2}^{\oplus 2}$. If we apply the universal coefficient theorem in Lemma 5.4 to $H_{P}^{n}\left(\mathrm{pt} ; \mathbb{Z}_{\phi}\right)$, then

$$
H_{D_{2 m}}^{1}\left(\mathrm{pt} ; \mathbb{Z}_{2}\right) \cong \mathbb{Z}_{2} \oplus \operatorname{Tor}\left(H_{D_{2 m}}^{2}\left(\mathrm{pt} ; \mathbb{Z}_{\phi}\right), \mathbb{Z}_{2}\right)
$$

Thus the consistency of these computations implies $H_{D_{2 m}}^{2}\left(\mathrm{pt} ; \mathbb{Z}_{\phi}\right) \cong \mathbb{Z}_{2}$. Based on this result, the second sequence in Lemma 5.5 suggests that $H_{D_{2 m}}^{3}\left(\mathrm{pt} ; \mathbb{Z}_{\phi}\right)$ is either $\mathbb{Z}_{2}^{\oplus 2}$ or $\mathbb{Z}_{4}$. If we apply the universal coefficient theorem to $H_{P}^{n}(\mathrm{pt} ; \mathbb{Z})$, then $H_{D_{2 m}}^{2}\left(\mathrm{pt} ; \mathbb{Z}_{2}\right) \cong \mathbb{Z}_{2}^{\oplus 3}$. If we compute this cohomology applying Lemma 5.4 to $H_{P}^{n}\left(\mathrm{pt} ; \mathbb{Z}_{\phi}\right)$, then

$$
H_{D_{2 m}}^{2}\left(\mathrm{pt} ; \mathbb{Z}_{2}\right) \cong \mathbb{Z}_{2} \oplus \operatorname{Tor}\left(H_{D_{2 m}}^{3}\left(\mathrm{pt} ; \mathbb{Z}_{\phi}\right), \mathbb{Z}_{2}\right)
$$

Therefore we conclude that $\left.H_{D_{2 m}}^{3}\left(\mathrm{pt} ; \mathbb{Z}_{\phi}\right), \mathbb{Z}_{2}\right) \cong \mathbb{Z}_{2}^{\oplus 2}$ by the consistency. In the other cases, a combined use of the two exact sequences in Lemma 5.5 determines the group $H_{P}^{n}\left(\mathrm{pt} ; \mathbb{Z}_{\phi}\right)$ without difficulty. 


\subsection{The proof of Theorems 1.5 and 1.6}

Theorems 1.5 and 1.6 again follow from case-by-case computations. To these cases, we can apply the methods in the proof of Theorems 1.1 and 1.3. However, in some cases, only the possibility of a cohomology group is suggested by an exact sequence. In this case, we apply an argument used in the proof of Lemma 5.6: We compute the cohomology with coefficients in $\mathbb{Z}_{2}$ applying the universal coefficient theorem to the result in Theorem 1.3. Then the consistency with Lemma 5.4 eventually determines the cohomology in question.

In the following, we carry out the computation in the case of p6m with $\phi=\phi_{2}$. Let $Y \subset T^{2}$ be the $D_{6}$-invariant subspace given in Section 4.5.

Lemma 5.7. The $D_{6}$-equivariant cohomology of $Y$ with coefficients in $\mathbb{Z}_{\phi_{2}}$ in low degrees is as follows:

\begin{tabular}{|c|c|c|c|c|}
\hline & $n=0$ & $n=1$ & $n=2$ & $n=3$ \\
\hline$H_{D_{6}}^{n}\left(Y ; \mathbb{Z}_{\phi_{2}}\right)$ & 0 & $\mathbb{Z}_{2}$ & $\mathbb{Z}_{2}^{\oplus 2}$ & $\mathbb{Z}_{2}^{\oplus 3}$ \\
\hline
\end{tabular}

Proof. To use the Mayer-Vietoris sequence, we cover $Y$ by $D_{6}$-invariant subspaces $U$ and $V$ which have the following $D_{6}$-equivariant homotopy equivalences

$$
U \simeq \mathrm{pt}, \quad V \simeq D_{6} / D_{2}, \quad U \cap V \simeq D_{6} / \mathbb{Z}_{2},
$$

where $D_{2}=\left\{1, C^{3}, \sigma_{1}, \sigma_{4}\right\} \subset D_{6}$ and $\mathbb{Z}_{2}=\left\{1, \sigma_{1}\right\} \subset D_{6}$. We see

$$
H_{D_{6}}^{n}\left(V ; \mathbb{Z}_{\phi_{2}}\right) \cong H_{D_{2}}^{n}\left(\mathrm{pt} ; \mathbb{Z}_{\phi_{2}}\right), \quad H_{D_{6}}^{n}\left(U \cap V ; \mathbb{Z}_{\phi_{2}}\right) \cong H_{\mathbb{Z}_{2}}^{n}\left(\mathrm{pt} ; \mathbb{Z}_{\phi_{1}}\right) .
$$

The equivariant cohomology groups in low degrees can be summarized as follows:

\begin{tabular}{|c|c|c|c|}
\hline$n=3$ & & $\mathbb{Z}_{2}^{\oplus 2} \oplus \mathbb{Z}_{2}^{\oplus 2}$ & $\mathbb{Z}_{2}$ \\
\hline$n=2$ & & $\mathbb{Z}_{2} \oplus \mathbb{Z}_{2}$ & 0 \\
\hline$n=1$ & & $\mathbb{Z}_{2} \oplus \mathbb{Z}_{2}$ & $\mathbb{Z}_{2}$ \\
\hline$n=0$ & & $0 \oplus 0$ & 0 \\
\hline & $H_{D_{6}}^{n}\left(Y ; \mathbb{Z}_{\phi_{2}}\right)$ & $H_{D_{6}}^{n}\left(U \sqcup V ; \mathbb{Z}_{\phi_{2}}\right)$ & $H_{D_{6}}^{n}\left(U \cap V ; \mathbb{Z}_{\phi_{2}}\right)$ \\
\hline
\end{tabular}

We have $H_{D_{6}}^{0}\left(Y ; \mathbb{Z}_{\phi_{2}}\right)=0$ clearly, and $H_{D_{6}}^{1}\left(Y ; \mathbb{Z}_{\phi_{2}}\right)$ is either $\mathbb{Z}_{2}$ or $\mathbb{Z}_{2}^{\oplus 2}$. Applying the universal coefficient theorem to Lemma 4.9 , we find $H_{D_{6}}^{0}\left(Y ; \mathbb{Z}_{2}\right) \cong \mathbb{Z}_{2}$. This result must be consistent with the computation of $H_{D_{6}}^{0}\left(Y ; \mathbb{Z}_{2}\right)$ by using Lemma 5.4 , which leads to $H_{D_{6}}^{1}\left(Y ; \mathbb{Z}_{\phi_{2}}\right) \cong \mathbb{Z}_{2}$. Solving the Mayer-Vietoris exact sequence, we then find $H_{D_{6}}^{2}\left(Y ; \mathbb{Z}_{\phi_{2}}\right) \cong \mathbb{Z}_{2}^{\oplus 2}$. We also find that $H_{D_{6}}^{3}\left(Y ; \mathbb{Z}_{\phi_{2}}\right)$ is either $\mathbb{Z}_{2}^{\oplus 3}$ or $\mathbb{Z}_{2}^{\oplus 4}$. Computing again the cohomology with $\mathbb{Z}_{2}$-coefficients in two ways, we conclude that $H_{D_{6}}^{3}\left(Y ; \mathbb{Z}_{\phi_{2}}\right) \cong \mathbb{Z}_{2}^{\oplus 3}$.

Lemma 5.8. There is an exact sequence of $D_{6}$-modules

$$
0 \rightarrow H^{1}\left(T^{2} ; \mathbb{Z}\right) \otimes \mathbb{Z}_{\phi_{2}} \rightarrow H^{1}(Y ; \mathbb{Z}) \otimes \mathbb{Z}_{\phi_{2}} \stackrel{\pi}{\rightarrow} \mathbb{Z}_{\phi_{0}} \rightarrow 0
$$

admitting a module homomorphism $s: \mathbb{Z}_{\phi_{0}} \rightarrow H^{1}(Y ; \mathbb{Z}) \otimes \mathbb{Z}_{\phi_{2}}$ such that $\pi \circ s=3$.

Proof. The proof of Lemma 4.12 can be adapted to this case.

Lemma 5.9. $H_{\text {group }}^{n}\left(D_{6} ; H^{1}\left(T^{2} ; \mathbb{Z}\right) \otimes \mathbb{Z}_{\phi_{2}}\right)=0$ for $n=0,1,2$.

Proof. We use the long exact sequence of group cohomology induced from the short exact sequence of coefficients. Notice that

$$
H_{\text {group }}^{n}\left(D_{6} ; H^{1}(Y ; \mathbb{Z}) \otimes \mathbb{Z}_{\phi_{2}}\right) \cong \tilde{H}_{D_{6}}^{n+1}\left(Y ; \mathbb{Z}_{\phi_{2}}\right) .
$$


The relevant cohomology can be summarized as follows:

\begin{tabular}{|c|c|c|c|}
\hline 2 & & $\mathbb{Z}_{2}$ & $\mathbb{Z}_{6}$ \\
\hline 1 & & $\mathbb{Z}_{2}$ & $\mathbb{Z}_{2}$ \\
\hline 0 & & 0 & 0 \\
\hline$n$ & $H_{\text {group }}^{n}\left(D_{6} ; H^{1}\left(T^{2}\right) \otimes \mathbb{Z}_{\phi_{2}}\right)$ & $H_{\text {group }}^{n}\left(D_{6} ; H^{1}(Y) \otimes \mathbb{Z}_{\phi_{2}}\right)$ & $H_{\text {group }}^{n}\left(D_{6} ; \mathbb{Z}_{\phi_{0}}\right)$ \\
\hline
\end{tabular}

By $s: \mathbb{Z}_{\phi_{0}} \rightarrow H^{1}(Y ; \mathbb{Z}) \otimes \mathbb{Z}_{\phi_{2}}$, the group cohomology is determined as stated.

Theorem 5.10 ( $\mathrm{p} 6 \mathrm{~m}$ with $\phi_{2}$ ). The $D_{6}$-equivariant cohomology of $T^{2}$ with coefficients in $\mathbb{Z}_{\phi_{2}}$ in low degrees is given as follows:

\begin{tabular}{|c|c|c|c|c|}
\hline & $n=0$ & $n=1$ & $n=2$ & $n=3$ \\
\hline$H_{D_{6}}^{n}\left(T^{2} ; \mathbb{Z}_{\phi_{2}}\right)$ & 0 & $\mathbb{Z}_{2}$ & $\mathbb{Z}_{2}$ & $\mathbb{Z}_{2}^{\oplus 3}$ \\
\hline
\end{tabular}

We also have: $F^{2} H_{D_{6}}^{3}\left(T^{2} ; \mathbb{Z}_{\phi_{2}}\right) \cong F^{3} H_{D_{6}}^{3}\left(T^{2} ; \mathbb{Z}_{\phi_{2}}\right) \cong \mathbb{Z}_{2}^{\oplus 2}$.

Proof. In the $E_{2}$-term of the Leray-Serre spectral sequence, we have the following identifications

$$
\begin{aligned}
& H_{\text {group }}^{n}\left(D_{6} ; H^{0}\left(T^{2} ; \mathbb{Z}\right) \otimes \mathbb{Z}_{\phi_{2}}\right) \cong H_{D_{6}}^{n}\left(\mathrm{pt} ; \mathbb{Z}_{\phi_{2}}\right), \\
& H_{\text {group }}^{n}\left(D_{6} ; H^{2}\left(T^{2} ; \mathbb{Z}\right) \otimes \mathbb{Z}_{\phi_{2}}\right) \cong H_{D_{6}}^{n}\left(\mathrm{pt} ; \mathbb{Z}_{\phi_{1}}\right) .
\end{aligned}
$$

We can summarize the $E_{2}$-terms as follows:

\begin{tabular}{|c|c|c|c|c|}
\hline$q=3$ & 0 & 0 & 0 & 0 \\
\hline$q=2$ & 0 & $\mathbb{Z}_{2}$ & & \\
\hline$q=1$ & 0 & 0 & 0 & \\
\hline$q=0$ & 0 & $\mathbb{Z}_{2}$ & $\mathbb{Z}_{2}$ & $\mathbb{Z}_{2}^{\oplus 2}$ \\
\hline$E_{2}^{p, q}$ & $p=0$ & $p=1$ & $p=2$ & $p=3$ \\
\hline
\end{tabular}

Because $E_{2}^{n, 0}$ must survive into the direct summand $H_{D_{6}}^{n}\left(\mathrm{pt} ; \mathbb{Z}_{\phi_{2}}\right)$ of the cohomology $H_{D_{6}}^{n}\left(T^{2} ; \mathbb{Z}_{\phi_{2}}\right)$, we get the degeneration $E_{2}^{p, q}=E_{\infty}^{p, q}$ for $p+q \leq 2$, and the relevant extension problems are readily solved. We also have $H_{D_{6}}^{3}\left(T^{2} ; \mathbb{Z}_{\phi_{2}}\right) \cong \mathbb{Z}_{2}^{\oplus 2} \oplus E_{\infty}^{1,2}$, where $E_{\infty}^{1,2} \subset E_{2}^{1,2}=\mathbb{Z}_{2}$ is either $\mathbb{Z}_{2}$ or 0 . By computing the cohomology with $\mathbb{Z}_{2}$-coefficients in two ways, we conclude that $E_{\infty}^{1,2}=E_{2}^{1,2}=\mathbb{Z}_{2}$.

\section{A The list of 2-dimensional space groups}

Here is a list of the lattices $\Pi$ and the point groups $P$ of the 2 -dimensional space groups $S$. In the nonsymmorphic case, the map $a: P \rightarrow \mathbb{R}^{2}$ in Section 2 is also presented.

\section{A.1 Oblique, rectangular and square lattices}

For p1, p2, p4, pm, pg, pmm, pmg, pgg, p4m and p4g, we can take the lattice $\Pi \subset \mathbb{R}^{2}$ to be the standard lattice $\Pi=\mathbb{Z}^{2}$.

- (p1) The point group is trivial.

- (p2) The point group $\mathbb{Z}_{2}=\left\langle C \mid C^{2}\right\rangle$ acts on $\Pi$ and $\mathbb{R}^{2}$ through the matrix

$$
C=\left(\begin{array}{cc}
-1 & 0 \\
0 & -1
\end{array}\right)
$$


- (p4) The point group $\mathbb{Z}_{4}=\left\langle C \mid C^{4}\right\rangle$ acts on $\Pi$ and $\mathbb{R}^{2}$ through

$$
C=\left(\begin{array}{cc}
0 & -1 \\
1 & 0
\end{array}\right) \text {. }
$$

- $(\mathrm{pm} / \mathrm{pg})$ The point group $D_{1}=\left\langle\sigma \mid \sigma^{2}\right\rangle$ acts on $\Pi$ and $\mathbb{R}^{2}$ through

$$
\left(\begin{array}{cc}
-1 & 0 \\
0 & 1
\end{array}\right)
$$

In the case of $\mathrm{pg}$, the map $a: D_{1} \rightarrow \mathbb{R}^{2}$ is given by

$$
a_{1}=\left(\begin{array}{l}
0 \\
0
\end{array}\right), \quad a_{\sigma}=\left(\begin{array}{c}
0 \\
1 / 2
\end{array}\right)
$$

- $(\mathrm{pmm} / \mathrm{pmg} / \mathrm{pgg})$ The point group is $D_{2} \cong \mathbb{Z}_{2} \times \mathbb{Z}_{2}$. We let the following matrices $\sigma_{x}$ and $\sigma_{y}$ generate $D_{2}$, and act on $\Pi$ and $\mathbb{R}^{2}$

$$
\sigma_{x}=\left(\begin{array}{cc}
-1 & 0 \\
0 & 1
\end{array}\right), \quad \sigma_{y}=\left(\begin{array}{cc}
1 & 0 \\
0 & -1
\end{array}\right)
$$

In the case of pmg, the map $a: D_{2} \rightarrow \mathbb{R}^{2}$ is given by

$$
a_{1}=\left(\begin{array}{l}
0 \\
0
\end{array}\right), \quad a_{\sigma_{x}}=\left(\begin{array}{c}
0 \\
1 / 2
\end{array}\right), \quad a_{\sigma_{y}}=\left(\begin{array}{l}
0 \\
0
\end{array}\right), \quad a_{\sigma_{x} \sigma_{y}}=\left(\begin{array}{c}
0 \\
1 / 2
\end{array}\right) .
$$

In the case of pgg, the map $a: D_{2} \rightarrow \mathbb{R}^{2}$ is given by

$$
a_{1}=\left(\begin{array}{l}
0 \\
0
\end{array}\right), \quad a_{\sigma_{x}}=\left(\begin{array}{c}
0 \\
1 / 2
\end{array}\right), \quad a_{\sigma_{y}}=\left(\begin{array}{c}
1 / 2 \\
0
\end{array}\right), \quad a_{\sigma_{x} \sigma_{y}}=\left(\begin{array}{c}
1 / 2 \\
1 / 2
\end{array}\right) .
$$

- (p4m/p4g) The point group is $D_{4}=\left\langle C_{4}, \sigma_{x} \mid C_{4}^{4}, \sigma_{x}^{2}, \sigma_{x} C_{4} \sigma_{x} C_{4}\right\rangle$, which acts on $\Pi$ and $\mathbb{R}^{2}$ through the following matrix presentation

$$
C_{4}=\left(\begin{array}{cc}
0 & -1 \\
1 & 0
\end{array}\right), \quad \sigma_{x}=\left(\begin{array}{cc}
-1 & 0 \\
0 & 1
\end{array}\right)
$$

In the case of $\mathrm{p} 4 \mathrm{~g}$, the map $a: D_{4} \rightarrow \mathbb{R}^{2}$ is as follows:

\begin{tabular}{|c|cccc|cccc|}
\hline$p$ & 1 & $C_{4}$ & $C_{4}^{2}$ & $C_{4}^{3}$ & $\sigma_{x}$ & $\sigma_{d}$ & $\sigma_{y}$ & $\sigma_{d}^{\prime}$ \\
\hline$a_{p}$ & {$\left[\begin{array}{c}0 \\
0\end{array}\right]$} & {$\left[\begin{array}{c}0 \\
\frac{1}{2}\end{array}\right]$} & {$\left[\begin{array}{c}\frac{1}{2} \\
\frac{1}{2}\end{array}\right]$} & {$\left[\begin{array}{c}\frac{1}{2} \\
0\end{array}\right]$} & {$\left[\begin{array}{c}0 \\
\frac{1}{2}\end{array}\right]$} & {$\left[\begin{array}{c}0 \\
0\end{array}\right]$} & {$\left[\begin{array}{c}\frac{1}{2} \\
0\end{array}\right]$} & {$\left[\begin{array}{c}\frac{1}{2} \\
\frac{1}{2}\end{array}\right]$} \\
\hline
\end{tabular}

In the above, we set $\sigma_{d}=\sigma_{x} C_{4}, \sigma_{y}=C_{4}^{2} \sigma_{x}$ and $\sigma_{d}^{\prime}=C_{4} \sigma_{x}$.

\section{A.2 Rhombic lattices}

For $\mathrm{cm}$ and $\mathrm{cmm}$, the lattice is $\Pi=\mathbb{Z} a \oplus \mathbb{Z} b \subset \mathbb{R}^{2}$, where

$$
a=\left(\begin{array}{l}
1 \\
1
\end{array}\right), \quad b=\left(\begin{array}{c}
-1 \\
1
\end{array}\right) .
$$

- (cm) The point group $D_{1}=\left\langle\sigma \mid \sigma^{2}\right\rangle$ acts on $\Pi$ and $\mathbb{R}^{2}$ by

$$
\sigma=\left(\begin{array}{cc}
-1 & 0 \\
0 & 1
\end{array}\right)
$$

- $(\mathrm{cmm})$ The point group is $D_{2} \cong \mathbb{Z}_{2} \times \mathbb{Z}_{2}$. The following matrices $\sigma_{x}$ and $\sigma_{y}$ generate $D_{2}$, and define the $D_{4}$-action on $\Pi$ and $\mathbb{R}^{2}$

$$
\sigma_{x}=\left(\begin{array}{cc}
-1 & 0 \\
0 & 1
\end{array}\right), \quad \sigma_{y}=\left(\begin{array}{cc}
1 & 0 \\
0 & -1
\end{array}\right)
$$




\section{A.3 Hexagonal lattices}

For p3, p6, p3m1, p31m and p6m, the lattice $\Pi=\mathbb{Z} a \oplus \mathbb{Z} b \subset \mathbb{R}^{2}$ is spanned by

$$
a=\left(\begin{array}{l}
1 \\
0
\end{array}\right), \quad b=\left(\begin{array}{c}
1 / 2 \\
\sqrt{3} / 2
\end{array}\right) .
$$

- (p3) The point group $\mathbb{Z}_{3}=\left\langle C \mid C^{3}\right\rangle$ acts on $\Pi$ and $\mathbb{R}^{2}$ through

$$
C=\left(\begin{array}{cc}
-1 / 2 & -\sqrt{3} / 2 \\
\sqrt{3} / 2 & -1 / 2
\end{array}\right)
$$

- (p6) The point group $\mathbb{Z}_{6}=\left\langle C \mid C^{6}\right\rangle$ acts on $\Pi$ and $\mathbb{R}^{2}$ through

$$
C=\left(\begin{array}{cc}
1 / 2 & -\sqrt{3} / 2 \\
\sqrt{3} / 2 & 1 / 2
\end{array}\right)
$$

- (p3m1) The point group is $D_{3}=\left\langle C, \sigma_{x} \mid C^{3}, \sigma_{x}^{2}, \sigma_{x} C \sigma_{x} C\right\rangle$. We let $D_{3}$ act on $\Pi$ and $\mathbb{R}^{2}$ through the inclusion $D_{3} \subset \mathrm{O}(2)$ given by

$$
C=\left(\begin{array}{cc}
-1 / 2 & -\sqrt{3} / 2 \\
\sqrt{3} / 2 & -1 / 2
\end{array}\right), \quad \sigma_{x}=\left(\begin{array}{cc}
-1 & 0 \\
0 & 1
\end{array}\right)
$$

- (p31m) The point group is $D_{3}=\left\langle C, \sigma_{y} \mid C^{3}, \sigma_{y}^{2}, \sigma_{y} C \sigma_{y} C\right\rangle$. We let $D_{3}$ act on $\Pi$ and $\mathbb{R}^{2}$ through the inclusion $D_{3} \subset \mathrm{O}(2)$ given by

$$
C=\left(\begin{array}{cc}
-1 / 2 & -\sqrt{3} / 2 \\
\sqrt{3} / 2 & -1 / 2
\end{array}\right), \quad \sigma_{y}=\left(\begin{array}{cc}
1 & 0 \\
0 & -1
\end{array}\right)
$$

- (p6m) The point group is $D_{6}=\left\langle C, \sigma_{1} \mid C^{6}, \sigma_{1}^{2}, \sigma_{1} C \sigma_{1} C\right\rangle$. We let $D_{6}$ act on $\Pi$ and $\mathbb{R}^{2}$ through the inclusion $D_{6} \subset \mathrm{O}(2)$ given by

$$
C=\left(\begin{array}{cc}
1 / 2 & -\sqrt{3} / 2 \\
\sqrt{3} / 2 & 1 / 2
\end{array}\right), \quad \sigma_{1}=\left(\begin{array}{cc}
1 & 0 \\
0 & -1
\end{array}\right)
$$

\section{Acknowledgements}

I would like to thank K. Shiozaki and M. Sato for valuable discussions. I would also thank G.C. Thiang, D. Tamaki, anonymous referees and an editor for helpful criticisms and comments. This work is supported by JSPS KAKENHI Grant Number JP15K04871.

\section{References}

[1] Adem A., Duman A.N., Gómez J.M., Cohomology of toroidal orbifold quotients, J. Algebra 344 (2011), 114-136, arXiv:1003.0435.

[2] Adem A., Ge J., Pan J., Petrosyan N., Compatible actions and cohomology of crystallographic groups, J. Algebra 320 (2008), 341-353, arXiv:0704.1823.

[3] Adem A., Pan J., Toroidal orbifolds, Gerbes and group cohomology, Trans. Amer. Math. Soc. 358 (2006), 3969-3983, math.AT/0406130.

[4] Bott R., Tu L.W., Differential forms in algebraic topology, Graduate Texts in Mathematics, Vol. 82, SpringerVerlag, New York - Berlin, 1982. 
[5] Donovan P., Karoubi M., Graded Brauer groups and $K$-theory with local coefficients, Inst. Hautes Études Sci. Publ. Math. (1970), 5-25.

[6] Dupont J.L., Curvature and characteristic classes, Lecture Notes in Math., Vol. 640, Springer-Verlag, Berlin New York, 1978.

[7] Freed D.S., Hopkins M.J., Teleman C., Loop groups and twisted K-theory I, J. Topol. 4 (2011), 737-798, arXiv:0711.1906.

[8] Freed D.S., Moore G.W., Twisted equivariant matter, Ann. Henri Poincaré 14 (2013), 1927-2023, arXiv:1208.5055.

[9] Gomi K., Equivariant smooth Deligne cohomology, Osaka J. Math. 42 (2005), 309-337, math.DG/0307373.

[10] Gomi K., A variant of $K$-theory and topological T-duality for real circle bundles, Comm. Math. Phys. 334 (2015), 923-975, arXiv:1310.8446.

[11] Hatcher A., Algebraic topology, Cambridge University Press, Cambridge, 2002.

[12] Hiller H., Crystallography and cohomology of groups, Amer. Math. Monthly 93 (1986), 765-779.

[13] Karoubi M., K-theory. An introduction, Grundlehren der Mathematischen Wissenschaften, Vol. 226, Springer-Verlag, Berlin - New York, 1978.

[14] Karpilovsky G., Projective representations of finite groups, Monographs and Textbooks in Pure and Applied Mathematics, Vol. 94, Marcel Dekker, Inc., New York, 1985.

[15] Kaufmann R.M., Khlebnikov S., Wehefritz-Kaufmann B., Projective representations from quantum enhanced graph symmetries, J. Phys. Conf. Ser. 597 (2015), 012048, 16 pages.

[16] Kaufmann R.M., Khlebnikov S., Wehefritz-Kaufmann B., Re-gauging groupoid, symmetries and degeneracies for graph Hamiltonians and applications to the gyroid wire network, Ann. Henri Poincaré 17 (2016), 1383-1414, arXiv:1208.3266.

[17] Kitaev A., Periodic table for topological insulators and superconductors, AIP Conf. Proc. 1134 (2009), 22-30, arXiv:0901.2686.

[18] Kubota Y., Notes on twisted equivariant K-theory for $\mathrm{C}^{*}$-algebras, Internat. J. Math. 27 (2016), 1650058, 28 pages, arXiv:1511.05312.

[19] May J.P., Cole M., Comezana G.R., Costenoble S.R., Elmendorf A.D., Greenlees J.P., Lewis L.G., Piacenza R.J., Triantafillou G., Waner S., Equivariant homotopy and cohomology theory, CBMS Regional Conference Series in Mathematics, Vol. 91, Amer. Math. Soc., Providence, RI, 1996.

[20] Newman M., Integral matrices, Pure and Applied Mathematics, Vol. 45, Academic Press, New York London, 1972.

[21] Rolfsen D., Knots and links, Mathematics Lecture Series, Vol. 7, Publish or Perish, Inc., Houston, TX, 1990.

[22] Rosenberg J., Continuous-trace algebras from the bundle theoretic point of view, J. Austral. Math. Soc. Ser. A 47 (1989), 368-381.

[23] Schattschneider D., The plane symmetry groups: their recognition and notation, Amer. Math. Monthly 85 (1978), 439-450.

[24] Schwarzenberger R.L.E., The 17 plane symmetry groups, Math. Gaz. 58 (1974), 123-131.

[25] Schwarzenberger R.L.E., Colour symmetry, Bull. London Math. Soc. 16 (1984), 209-240.

[26] Shiozaki K., Sato M., Gomi K., $Z_{2}$-topology in nonsymmorphic crystalline insulators: Mobius twist in surface states, Phys. Rev. B 91 (2015), 155120, 9 pages, arXiv:1502.03265.

[27] Shiozaki K., Sato M., Gomi K., Topology of nonsymmorphic crystalline insulators and superconductors, Phys. Rev. B 93 (2016), 195413, 28 pages, arXiv:1511.01463.

[28] Shiozaki K., Sato M., Gomi K., Topological crystalline materials - general formulation and wallpaper group classification, arXiv:1701.08725.

[29] Thiang G.C., On the K-theoretic classification of topological phases of matter, Ann. Henri Poincaré 17 (2016), 757-794, arXiv:1406.7366. 